August 4, 2018

\title{
Boundary Conditions in Rational Conformal Field Theories
}

\author{
Roger E. Behrend \\ C.N. Yang Institute for Theoretical Physics \\ State University of New York \\ Stony Brook, NY 11794-3840, USA \\ Paul A. Pearce \\ Department of Mathematics and Statistics \\ University of Melbourne \\ Parkville, Victoria 3052, Australia \\ Valentina B. Petkova \\ Arnold Sommerfeld Institute for Mathematical Physics \\ TU Clausthal, Leibnizstr. 10 \\ D-38678 Clausthal-Zellerfeld, Germany \\ Jean-Bernard Zuber \\ Service de Physique Théorique \\ CEA-Saclay \\ 91191 Gif-sur-Yvette Cedex, France
}

\begin{abstract}
We develop further the theory of Rational Conformal Field Theories (RCFTs) on a cylinder with specified boundary conditions emphasizing the role of a triplet of algebras: the Verlinde, graph fusion and Pasquier algebras. We show that solving Cardy's equation, expressing consistency of a RCFT on a cylinder, is equivalent to finding integer valued matrix representations of the Verlinde algebra. These matrices allow us to naturally associate a graph $G$ to each RCFT such that the conformal boundary conditions are labelled by the nodes of $G$. This approach is carried to completion for $s l(2)$ theories leading to complete sets of conformal boundary conditions, their associated cylinder partition functions and the $A-D-E$ classification. We also review the current status for WZW $\operatorname{sl}(3)$ theories. Finally, a systematic generalization of the formalism of Cardy-Lewellen is developed to allow for multiplicities arising from more general representations of the Verlinde algebra. We obtain information on the bulk-boundary coefficients and reproduce the relevant algebraic structures from the sewing constraints.
\end{abstract}

\footnotetext{
${ }^{1}$ permanent address: Institute for Nuclear Research and Nuclear Energy, Tzarigradsko Chaussee 72, 1784 Sofia, Bulgaria
} 


\section{Introduction}

\section{$1.1 \quad$ History and motivation}

The subject of boundary conformal field theory has a fairly long history. It was born more than ten years ago, in parallel work on open string theory [1, 2, 3, 4, 5, 6, 7, 8] and on conformal field theories (CFTs) describing critical systems with boundaries [9]. The work of Cardy 10 was a landmark, leading to the unification of methods, to the introduction of important concepts such as boundary conformal fields and to the systematic investigation of their properties and couplings [11, 12]. The subject remained dormant for some time, in spite of some activity motivated again by string theory [13, 14, 15] and of beautiful applications to the Kondo problem 116, 17]. Lately, the subject has undergone a revival of interest in connection with various problems. On the one hand, work on boundary conditions in integrable field theories and boundary flows [18, 19, 20] and on quantum impurities [21, 22] motivated a closer look at boundary CFT. On the other hand, within statistical mechanics, integrable Boltzmann weights satisfying the so-called Boundary Yang-Baxter Equation (BYBE) were constructed in lattice models [23, 24]. Finally new progress in string theory was another reason to reconsider the problem. Generalizations of D-branes as boundary conditions in CFT have been studied by several groups [25, 26, 27, 28, 29, 30].

In the present work, we want to reconsider several issues in the discussion of boundary conditions in (rational) conformal field theories: what are the general boundary conditions that may be imposed, what are the structure constants of the bulk and boundary fields in the presence of these boundary conditions. These are the basic questions that we want to address. The methods that we use are not essentially new, but are based on the systematic exploitation of the work of Cardy and Lewellen [10, 11, 12].

Among the main results of this paper:

- We establish a connection between the classification of boundary conditions and the classification of integer valued representations of the fusion algebra. A preliminary account of this result was given in [31, 32]. In the same vein, we show that it is natural to associate graphs to these problems. In particular, an ADE classification of boundary conditions for Wess-Zumino-Witten (WZW) and minimal $s l(2)$ theories emerges in a natural and simple way. A discussion of the state of the art for $\operatorname{sl}(3)$ models is also included.

- We point out the deep connections between the features of conformal field theory in the bulk and in the presence of boundaries. The classification of the latter has some bearing on the classification of the bulk properties (modular invariants etc). This is not a new observation. In particular, in string theory many connections are known to exist between open and closed string sectors, but it seems that the point had not been stressed enough. A triplet of algebras, specifically the graph fusion algebra and its dual, the Pasquier algebra, appears naturally in our discussion, along with the Verlinde 
algebra.

- We reanalyse in a systematic way the couplings (structure constants) of fields in the presence of boundaries and the equations they satisfy, generalising the formalism of Cardy-Lewellen to accomodate the appearance of nontrivial multiplicities. In the diagonal cases we find a direct relation between the chiral duality identities of Moore and Seiberg and the basic sewing relations of the boundary CFT. The main point is the observation that the bulk boundary coefficients in the diagonal case essentially coincide with the matrices $S(j)$ of modular transformations of torus 1-point functions. In this way the two basic bulk-boundary equations [11, 12 are shown to be equivalent to the torus duality identity of [33.

Some more particular results include the extension of Cardy's equation to non-specialized characters, thus lifting an ambiguity in the original derivation, the proof of uniqueness of boundary conditions for $\widehat{s l}(2)$ WZW and minimal models and $\widehat{s l}(N)_{1}$ models, the clarification of the role of the graph algebra and the recovery of this algebra along with its dual, the Pasquier algebra, from the boundary sewing constraints.

\subsection{Background on bulk CFT}

In this paper, we are only concerned with Rational Conformal Field Theories (RCFTs). We first establish notations etc. In the study of a RCFT, one first specifies a chiral algebra $\mathcal{A}$. It is the Virasoro algebra or one of its extensions: current algebra, $\mathcal{W}$ algebra etc. The generators of this algebra will be denoted generically $W_{n}$ and include the Virasoro generators $L_{n}$. At a given level, the theory is rational, i.e. $\mathcal{A}$ has only a finite set $\mathcal{I}$ of irreducible representations $\mathcal{V}_{i}, i \in \mathcal{I}$. The label $i^{*}$ indexes the representation conjugate to $i$, and $i=1$ refers to the identity (or vacuum) representation. We also suppose that the characters $\chi_{i}(q)=\operatorname{tr}_{\mathcal{V}_{i}} q^{L_{0}-\frac{c}{24}}$ of these representations, the matrix $S$ of modular transformations of the $\chi$ 's and the fusion coefficients $N_{i j}{ }^{k}$ of the $\mathcal{V}$ 's are all known. The matrix $S_{i j}$ is symmetric and unitary and satisfies $S^{2}=C$, where $C$ is the conjugation matrix $C_{i j}=\delta_{j i^{*}}$. The fusion coefficients are assumed to be given in terms of $S$ by the Verlinde formula [34]

$$
N_{i j}{ }^{k}=\sum_{l \in \mathcal{I}} \frac{S_{i l} S_{j l} S_{k l}^{*}}{S_{1 l}},
$$

an assumption that rules out some cases of RCFTs.

A physical conformal theory is then defined by a collection of bulk and boundary fields and their 3-point couplings (OPE Coefficients). In particular, the spectrum of bulk fields is described by the finite set Spec of pairs $(j, \bar{j})$ of representations, possibly appearing with some multiplicities $N_{j \bar{j}}$, of the left and right copies of $\mathcal{A}$, such that the Hilbert space of the theory on an infinitely long cylinder reads

$$
\mathcal{H}=\oplus_{(j, \bar{j}) \in \operatorname{Spec}} \mathcal{V}_{j} \otimes \overline{\mathcal{V}}_{\bar{j}}
$$


with the same multiplicities $N_{j, \bar{j}}$. The modular invariant torus partition function

$$
Z_{\text {torus }}=\sum_{j, \bar{j}} N_{j \bar{j}} \chi_{j}(q)\left(\chi_{\bar{j}}(q)\right)^{*}
$$

is a convenient way to encode this information. The finite subset $\mathcal{E}$ of labels of elements of the spectrum that are left-right symmetric will play a central role in the following

$$
\mathcal{E}=\{j \mid(j, \bar{j}=j) \in \operatorname{Spec}\}
$$

and will be called the set of exponents of the theory. Recall that these exponents may come with some multiplicities. To distinguish them as different elements of the set $\mathcal{E}$ a second index will be often added, i.e., $(j, \alpha) \in \mathcal{E}$, for $j \in \mathcal{I}$.

In terms of all these data, one is in principle able to compute exactly all correlation functions of the CFT on an arbitrary 2D surface, with or without boundaries [35, 33, 12]. These data, however, are subject to consistency constraints: single-valuedness of $n$-point functions on the plane, modular invariance of the torus or annulus partition function, etc, all rooted in the locality properties of the theory. In this paper, we shall reexamine the conditions that stem from surfaces with boundaries (half-plane or disk, cylinder or annulus) and explore their consequences.

For later reference, let us also recall that RCFTs fall in two classes. In the first class ("type I"), the Hilbert space (1.2) is a diagonal sum of representations of a larger, "extended", algebra $\mathcal{A}^{\prime} \supseteq \mathcal{A}$. Accordingly, the partition function (1.3) is a sum of squares of sums of characters

$$
Z=\sum_{\text {blocks } B}\left|\sum_{i \in B} \chi_{i}\right|^{2}
$$

The second class ("type II") is obtained from the first by letting an automorphism $\zeta$ of the fusion rules of the extended algebra $\mathcal{A}^{\prime}$ act on the right components, thus resulting in a non-block-diagonal partition function

$$
Z=\sum_{B}\left(\sum_{i \in B} \chi_{i}\right)\left(\sum_{j \in \zeta(B)} \bar{\chi}_{j}\right)
$$

For example, in the classical case of $s l(2)$ theories, classified by $A-D-E$ Dynkin diagrams, the $A, D_{2 p}, E_{6}$ and $E_{8}$ cases are of the first type, whereas the $D_{2 p+1}$ and $E_{7}$ are obtained respectively from the $A_{4 p-1}$ and $D_{10}$ cases by a $\mathbb{Z}_{2}$ automorphism of their fusion rules. We shall see below that the study of boundary conditions on a cylinder has some bearing on these expressions of torus partition functions.

\section{Cardy Equation and Verlinde Algebra}




\subsection{Boundary states}

As discussed in [36], on the boundary of a domain such as the upper half plane or a semiinfinite cylinder, one must impose a continuity condition of the form

$$
T(z)=\left.\bar{T}(\bar{z})\right|_{z=\bar{z}} \quad W(z)=\left.\bar{W}(\bar{z})\right|_{z=\bar{z}}
$$

While the first of these conditions has the direct physical meaning of the absence of energymomentum flow across the boundary, or the preservation of the real boundary by diffeomorphisms, the condition(s) on the other $W$ may be generalized to incorporate a possible "gluing automorphism" 37, 25, 27]

$$
W(z)=\left.\Omega \bar{W}(\bar{z})\right|_{z=\bar{z}} .
$$

A semi-annular domain in the upper half-plane may be conformally mapped into an annulus in the complex plane by $\zeta=\exp (-2 i \pi w / T), w=\frac{L}{\pi} \log z$. Then as shown by Ishibashi [2] and Cardy [10], the boundary condition becomes

$$
\zeta^{2} T(\zeta)=\bar{\zeta}^{2} \bar{T}(\bar{\zeta}) \quad \zeta^{s_{W}} W(\zeta)=(-\bar{\zeta})^{s_{W}} \bar{W}(\bar{\zeta}) \quad \text { for }|\zeta|=1 \quad \text { and }|\zeta|=e^{2 \pi L / T}
$$

where $s_{W}$ denotes the spin of $W$, or more generally

$$
\zeta^{s_{W}} W(\zeta)=(-\bar{\zeta})^{s_{W}} \Omega \bar{W}(\bar{\zeta})
$$

Through radial quantization, this translates into a condition on boundary states $|a\rangle_{\Omega}$

$$
\left(W_{n}-(-1)^{s_{W}} \Omega\left(\bar{W}_{-n}\right)\right)|a\rangle_{\Omega}=0 .
$$

This includes in particular the condition that

$$
\left(L_{n}-\bar{L}_{-n}\right)|a\rangle_{\Omega}=0
$$

assuming that the automorphism $\Omega$ keeps invariant the Virasoro generators. For the central charge operator we have $(k-\bar{k})|a\rangle_{\Omega}=0$.

Solutions to this linear system are spanned by special states called Ishibashi states [2], labelled by the finite set $\mathcal{E}_{\Omega}=\{j \mid(j, \bar{j}=\omega(j)) \in$ Spec $\}$, where $\omega$ depends in particular on $\Omega$. To see this, let us consider first the simpler equation (2.5) in the case when $\mathcal{A}$ is the Virasoro algebra and $\Omega$ is trivial. Then observe that one may solve (2.5) in each component of (1.2) independently, as these spaces are invariant under the action of the two copies of $\mathcal{A}$. Now we recall that any state $A=\sum_{n, \bar{n}} a_{n, \bar{n}}|j, n\rangle \otimes|\bar{j}, \bar{n}\rangle$ in $\mathcal{V}_{j} \otimes \mathcal{V}_{\bar{j}}$ is in one-toone correspondence with a homomorphism $X_{A}=\sum_{n, \bar{n}} a_{n, \bar{n}}|j, n\rangle\langle\bar{j}, \bar{n}|$ of $\mathcal{V}_{\bar{j}}$ into $\mathcal{V}_{j}$. This uses the scalar product in $\mathcal{V}_{\bar{j}}$. Since $L_{-n}=L_{n}^{\dagger}$ for that scalar product, (2.5) implies that $L_{n} X_{A}=X_{A} L_{n}$, i.e. that $X_{A}$ intertwines the action of $L_{n}$ in the two representations $\mathcal{V}_{j}$ and $\mathcal{V}_{\bar{j}}$ of the Virasoro algebra. As these two representations are irreducible, they must be equivalent, which by our convention on the labelling of representations, means that $j=\bar{j}$. 
Thus the only non-vanishing components of $A$ in $\mathcal{H}$ are in diagonal products $\mathcal{V}_{j} \otimes \mathcal{V}_{j}$ and in each one, $X_{A}$ is proportional to the projector $P_{j}=\sum_{n}|j, n\rangle\langle j, n|$. To fix the normalization we choose $X_{A}=P_{j}$ and the corresponding Ishibashi state is denoted $\left.|j\rangle\right\rangle$. This completes the proof that there is an independent boundary state $|j\rangle\rangle$ for each element of the set $\mathcal{E}=\{j \mid(j, \bar{j}=j) \in$ Spec $\}$.

The argument is a formal extension of the proof, based on the Schur lemma, of the existence and uniqueness of an invariant in the tensor product of finite dimensional representations. It extends to the odd spin $s_{W}$ case (2.4). We have to use the fact that $W_{n}^{\dagger}=(-1)^{s_{W}} U^{-1} W_{-n} U$ with respect to a bilinear (or hermitian) form where $U$ is a unitary (or antiunitary) operator. One exploits the same definition of the homomorphism $X_{A}: \mathcal{V}_{j^{\prime}} \rightarrow \mathcal{V}_{j}$, now $\mathcal{V}_{j^{\prime}}, \mathcal{V}_{j}$ being highest weight representations of the chiral algebra $\mathcal{A}$ generated by $W_{n}$. However $X_{A}$ corresponds to states in $\mathcal{V}_{j} \otimes U_{\Omega} U \mathcal{V}_{j^{\prime}}$, where $U_{\Omega}$ is a unitary (antiunitary) operator implementing the automorphism $\Omega\left(W_{n}\right)=U_{\Omega} W_{n} U_{\Omega}^{-1}$. The equation (2.4) leads to $W_{n} X_{A}=X_{A} W_{n}$ again with the result $j^{\prime}=j$ and $X_{A}=P_{j}$ while the Ishibashi states are given by $|j\rangle_{\Omega}=\sum_{n}|j, n\rangle \otimes U_{\Omega} U|j, n\rangle$.

The operator $U$ is in general non-trivial, e.g., for the $\widehat{s l}(N)_{k}$ WZW theories $W_{n}^{\dagger}=$ $\bar{w}\left(W_{-n}\right)$ where $\bar{w}$ is the horizontal projection of the Chevalley involution of the affine algebra [38], i.e., it is determined by $\bar{w}\left(e^{\alpha_{i}}\right)=-f^{\alpha_{i}}, \bar{w}\left(f^{\alpha_{i}}\right)=-e^{\alpha_{i}}$, for the simple roots $\alpha_{i}$ of $\operatorname{sl}(N)$, where $e^{\alpha} / f^{\alpha}$ are raising/lowering operators respectively, and for the Cartan generators $\bar{w}\left(h^{i}\right)=-h^{i}, i=1,2, \ldots, N-1$. The pair $(j, \omega(j))$ characterising $\mathcal{E}_{\Omega}$ refers to the eigenvalues of $h^{i}$ on the first term in $\left.|j\rangle\right\rangle_{\Omega}$. If $\Omega$ is the identity, then $\omega(j)=-j$. If $\Omega=w_{0}$, where $w_{0}$ represents the longest element of the Weyl group, then $w_{0}(j)=-j^{*}$ and hence $\omega(j)=$ $w_{0} \bar{w}(j)=j^{*}$. On the other hand $\omega(j)=j$ for $\Omega$ coinciding with the Chevalley automorphism $\Omega=\bar{w}$. In the last two of these examples we can identify $\overline{\mathcal{V}}_{\bar{j}}=U_{\Omega} U \mathcal{V}_{j}$ with a highest weight module $\mathcal{V}_{\omega(j)}, \omega(j) \in \mathcal{I}$. It should be stressed that all these automorphisms $\Omega$ keep invariant the Sugawara Virasoro generators so the condition (2.5) is also satisfied on the corresponding Ishibashi states.

We shall hereafter drop the explicit dependence on $\Omega$.

In fact we still have to define a norm (or a scalar product) on boundary states, in particular on the Ishibashi states. We have to face two difficulties. First because of the infinite dimension of the representation $\mathcal{V}_{j}$, the most naive norm, proportional to $\operatorname{tr} P_{j}$, would be infinite. The second problem concerns non-unitary representations. In such cases, the hermitian form on $\mathcal{V}_{j}$ is not positive definite, and we may encounter signs in the norm of states.

The first problem requires some regularization of the naive norm. Let $\tilde{q}^{\frac{1}{2}}=e^{\frac{-\pi i}{\tau}}$ be a real number, $0<\tilde{q}<1$. Then $\left\langle\left\langle j\left|\tilde{q}^{\frac{1}{2}\left(L_{0}+\bar{L}_{0}-\frac{c}{12}\right)}\right| j\right\rangle\right\rangle=\operatorname{tr} P_{j} \tilde{q}^{L_{0}-\frac{c}{24}}=\chi_{j}(\tilde{q})$. We write in general, allowing some multiplicity $\alpha=1, \cdots, N_{j j}$ for the representations:

$$
\left\langle\left\langle j^{\prime}, \alpha^{\prime}\left|\tilde{q}^{\frac{1}{2}\left(L_{0}+\bar{L}_{0}-\frac{c}{12}\right)}\right| j, \alpha\right\rangle\right\rangle=\delta_{j j^{\prime}} \delta_{\alpha \alpha^{\prime}} \chi_{j}(\tilde{q}) .
$$

\footnotetext{
${ }^{2}$ Many thanks to G. Watts (private communication) to whom we owe this elegant derivation. Some elements had appeared already in M. Bauer's PhD thesis (1989).
} 
The norm of $|j\rangle\rangle$ should then be some renormalized version of the $\tilde{q} \rightarrow 1$ limit of (2.6) [2, 10], i.e., of the limit in which $q=e^{2 \pi i \tau}$, the modular transform of $\tilde{q}$, tends to 0 . In unitary theories, a new scalar product on boundary states may be defined according to

$$
\begin{aligned}
\left\langle\left\langle j \alpha \| j^{\prime} \alpha^{\prime}\right\rangle\right\rangle & =\lim _{\tilde{q} \rightarrow 1} q^{\frac{c}{24}}\left\langle\left\langle j^{\prime}, \alpha^{\prime}\left|\tilde{q}^{\frac{1}{2}\left(L_{0}+\bar{L}_{0}-\frac{c}{12}\right)}\right| j, \alpha\right\rangle\right\rangle \\
& =\delta_{j j^{\prime}} \delta_{\alpha \alpha^{\prime}} \lim _{q \rightarrow 0} q^{\frac{c}{24}} \chi_{j}(\tilde{q})=\delta_{j j^{\prime}} \delta_{\alpha \alpha^{\prime}} S_{1 j},
\end{aligned}
$$

where we have used the fact that in a unitary theory, the leading character in the $q \rightarrow 0$ limit is that of the identity operator $\chi_{1}(q) \approx q^{-\frac{c}{24}}$. Note that $S_{j 1}$ is, up to a factor $1 / S_{11}$, the quantum dimension of the representation $j$, a positive number. Thus in unitary theories the normalization chosen for $X_{j}$ is such that the states $\left.|j\rangle\right\rangle$ are orthogonal for the scalar product (2.7), with a square norm equal to $S_{1 j}$. Although in non-unitary theories the limit $q \rightarrow 0$ in (2.7) does not exist in general, due to the existence of representations of conformal weight $h_{i}<0$ that will dominate that limit, we may still define the norm by the same formula as (2.7). Alternatively, if $j_{0}$ denotes the unique representation of smallest conformal weight $h_{j_{0}}<0$ belonging to $\mathcal{E}$, and $c_{\mathrm{eff}}:=c-24 h_{j_{0}}$, then we may define

$$
\left\langle\left\langle j \alpha \| j^{\prime} \alpha^{\prime}\right\rangle\right\rangle=\delta_{j j^{\prime}} \delta_{\alpha \alpha^{\prime}} \frac{S_{1 j}}{S_{j_{0 j}}} \lim _{q \rightarrow 0} q^{\frac{c_{\text {eff }}}{24}} \chi_{j}(\tilde{q}) .
$$

In all cases we thus have

$$
\left\langle\left\langle j \alpha \| j^{\prime} \alpha^{\prime}\right\rangle\right\rangle=\delta_{j j^{\prime}} \delta_{\alpha \alpha^{\prime}} S_{1 j}
$$

which is now of indefinite sign. In the sequel, we use more compact notations and the multiplicity label $\alpha$ will be implicit when referring to $j \in \mathcal{E}$.

The most general boundary state $|a\rangle$ satisfying condition (2.4) must be a linear combination of these Ishibashi states, which, for later convenience, we write as

$$
\left.|a\rangle=\sum_{j \in \mathcal{E}} \frac{\psi_{a}^{j}}{\sqrt{S_{1 j}}}|j\rangle\right\rangle .
$$

We denote by $\mathcal{V}=\{a\}$ the set labelling the boundary states. We assume that an involution $a \rightarrow a^{*}$ in the set $\mathcal{V}$ is defined and that $\psi_{a^{*}}^{j}=\psi_{a}^{j^{*}}=\left(\psi_{a}^{j}\right)^{*}$, where $j \rightarrow j^{*}$ is an involution in $\mathcal{E}$ (in general $(j, \alpha) \rightarrow\left(j^{*}, \alpha^{*}\right)$, see Appendix B for examples). We define conjugate states as

$$
\langle b|:=\sum_{j \in \mathcal{E}}\left\langle\langle j| \frac{\psi_{b^{*}}^{j}}{\sqrt{S_{1 j}}} .\right.
$$

As explained in [25], this conjugate state may be regarded as resulting from the action of an antilinear CPT operation. As a consequence

$$
\langle b \| a\rangle=\sum_{j \in \mathcal{E}} \frac{\psi_{a}^{j}\left(\psi_{b}^{j}\right)^{*}}{S_{1 j}}\langle\langle j \| j\rangle\rangle=\sum_{j \in \mathcal{E}} \psi_{a}^{j}\left(\psi_{b}^{j}\right)^{*}
$$


so that the orthonormality of the boundary states is equivalent to that of the $\psi$ 's.

In some cases, such as in the computation of partition functions involving the specialised characters in the next section, it is sufficient to impose only the Virasoro condition (2.5) on the boundary states. Then the sum in (2.10), when interpreted in terms of Ishibashi states pertaining to some extended symmetry, may include states $|j\rangle\rangle_{\Omega}$ with different $\Omega$. For example, in the minimal $\widehat{s l}(2)$ models when multiplicities occur in $\mathcal{E}$, one can build the Ishibashi states using the Coulomb gas realisation with $\mathcal{A}=\widehat{u}(1)$. Then there are two choices of $\Omega$ keeping $L_{n}$ invariant. This allows, in particular, the construction of two different Ishibashi states with the same value of the scaling dimension, i.e., the explicit resolution of the degeneracy of states denoted $|j, \alpha\rangle\rangle$ above. Such mixtures of Ishibashi states may be used in determining the boundary states of the non-diagonal $\left(A, D_{\text {even }}\right)$ models.

\subsection{The Cardy equation}

We now consider a conformal field theory on a finite cylinder. Following Cardy, the partition function may be expressed in two alternative ways. Regarded as resulting from the evolution of the system between boundary states $a$ and $b$ under the action of the Hamiltonian on (i.e. the translation operator along) the cylinder, it is

$$
Z_{b \mid a}=\left\langle b\left|\tilde{q}^{\frac{1}{2}\left(L_{0}+\bar{L}_{0}-\frac{c}{12}\right)}\right| a\right\rangle
$$

where $\tilde{q}^{\frac{1}{2}}=e^{-2 \pi \frac{L}{T}}$ describes the aspect-ratio of the cylinder of period $T$ and length $L$ as

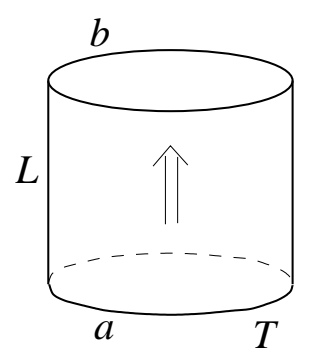

(a)

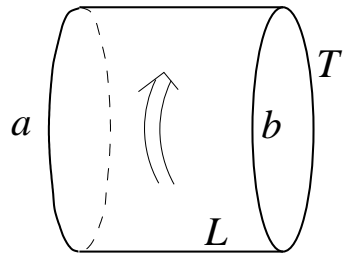

(b)

Figure 1: The two computations of the partition function $Z_{b \mid a}$ : (a) on the cylinder, between the boundary states $a$ and $b,(\mathrm{~b})$ as a periodic time evolution on the strip, with boundary conditions $a$ and $b$.

in Figure 1. Decomposing the boundary states on the Ishibashi basis and using (2.6), one obtains

$$
Z_{b \mid a}=\sum_{j \in \mathcal{E}} \psi_{a}^{j}\left(\psi_{b}^{j}\right)^{*} \frac{\chi_{j}(\tilde{q})}{S_{j 1}}
$$

where the states $|j\rangle\rangle$ are admissible Ishibashi states of the system, i.e., the label $j$ runs over the set $\mathcal{E}$. 
On the other hand, $Z_{b \mid a}$ may be regarded as resulting from the periodic "time" evolution under the action of the translation operator along the finite width strip in the presence of boundary conditions $a$ and $b$. The latter manifest themselves only in the nature of the Hilbert space $\mathcal{H}_{b a}$ and its decomposition into representations of a single chiral algebra: $\mathcal{H}_{b a}=$ $\oplus_{i} n_{i b}{ }^{a} \mathcal{V}_{i}$ with non-negative integer multiplicities $n_{i b}{ }^{a}$. If $q=e^{-\pi \frac{T}{L}}, Z_{b \mid a}$ is a linear form in the characters

$$
Z_{b \mid a}=\sum_{i \in \mathcal{I}} \chi_{i}(q) n_{i b}{ }^{a}
$$

We choose to write the modular transformation of characters in the form $\chi_{i}(q)=$ $\sum_{j} S_{i j} \chi_{j}(\tilde{q})$, hence $\chi_{j}(\tilde{q})=\sum_{i} S_{j i^{*}} \chi_{i}(q)$. Provided that specialized characters $\chi_{i}(q)$ are considered, this complex conjugation is immaterial, since $\chi_{i}(q)=\chi_{i^{*}}(q)$. We shall, however, make later use of unspecialized characters (Appendix A), for which it does matter. With this convention, and assuming for the time being the independence of characters, the two expressions (2.14) and (2.15) are consistent provided

$$
n_{i a}{ }^{b}=\sum_{j \in \mathcal{E}} \frac{S_{i j}}{S_{1 j}} \psi_{a}^{j}\left(\psi_{b}^{j}\right)^{*} .
$$

In the sequel we will refer to this as the Cardy equation. In the left hand side, we have used the first of the following symmetries

$$
n_{i a}{ }^{b}=n_{i^{*} b}{ }^{a}=n_{i b^{*}}{ }^{*}
$$

which follow from the properties of the modular matrix and of the coefficients $\psi_{a}^{j}$.

The boundary states $|a\rangle,|b\rangle$, are thus such that $n_{i a}{ }^{b}$ is a non-negative integer. Uniqueness of the vacuum implies $n_{1 a}{ }^{b} \leq 1$. The Cardy equation (2.16) is a non-linear constraint on the components of boundary states $|a\rangle$ and $|b\rangle$ on the basis of Ishibashi states. Note also that it implies that $\sum_{i} n_{i b}{ }^{a} S_{j i}$ vanishes if $j \notin \mathcal{E}$ and, except in cases with multiplicities, must factorize into a product of contributions of the $a$ and $b$ boundary states, a non-trivial constraint. Still, these constraints seem difficult to solve in full generality.

Before we proceed, we have to pause on the question of independence of characters. In general, it is not true that specialized characters such as those that we have been using so far, are linearly independent. For instance, complex conjugate representations $i$ and $i^{*}$ give rise to the same character $\chi_{i}(q)$. Unfortunately, little is known about unspecialized characters for general chiral algebras, beside the case of affine algebras. In Appendix A, we show that in the case of rational conformal field theories with a current algebra, the previous discussion may indeed be repeated if the energy momentum tensor of the theory has been modified in such a way that unspecialized characters appear. Then using the known modular transformations of the latter [38], one derives (2.16). We shall therefore assume that (2.16) holds true for general RCFT.

We now return to the Cardy equation (2.16), and assume that we have found an or- 
thonormal set of boundary states, i.e. satisfying

$$
\sum_{j \in \mathcal{E}} \psi_{a}^{j}\left(\psi_{b}^{j}\right)^{*}=\delta_{a b}
$$

Moreover we make the stronger assumption that we have found a complete set of such states, i.e. satisfying

$$
\sum_{a} \psi_{a}^{j}\left(\psi_{a}^{j^{\prime}}\right)^{*}=\delta_{j j^{\prime}}
$$

(Note that this implies that the number of these boundary states must be equal to the cardinality of $\mathcal{E}$ ).

Finally we recall that the ratios $S_{i j} / S_{1 j}$, for a fixed $j \in \mathcal{I}$, form a one-dimensional representation of the fusion algebra, as a consequence of the Verlinde formula (1.1):

$$
\frac{S_{i_{1} j}}{S_{1 j}} \frac{S_{i_{2} j}}{S_{1 j}}=\sum_{i_{3} \in \mathcal{I}} N_{i_{1} i_{2}} i_{3} \frac{S_{i_{3} j}}{S_{1 j}} .
$$

It follows from (2.19), (2.20) that the matrices $n_{i}$, defined by

$$
\left(n_{i}\right)_{a}{ }^{b}=n_{i a}{ }^{b} \quad i \in \mathcal{I}
$$

also satisfy the (commuting) fusion algebra

$$
n_{i_{1}} n_{i_{2}}=\sum_{i_{3} \in \mathcal{I}} N_{i_{1} i_{2}}^{{ }^{i}} n_{i_{3}}
$$

By (2.18), $n_{1}=I$, the unit matrix, and by (2.17), $n_{i^{*}}=n_{i}^{T}$.

Conversely, given a set of matrices with non-negative integer elements, satisfying $n_{i^{*}}=$ $n_{i}^{T}, n_{1}=I$ and the fusion algebra, they form a commuting set, and thus each $n_{i}$ commutes with its transpose. These matrices are thus normal matrices that may be diagonalized in an orthonormal basis. Their eigenvalues are of the form $S_{i j} / S_{1 j}$ for some $j$, and they may thus be written in the form (2.16). If one pretends to determine the spectrum $\mathcal{E}$ from the $n$ 's, one has to impose also that $j=1$ appears only once in $\mathcal{E}$, as a manifestation of the uniqueness of the vacuum.

We thus conclude that the search for orthonormal and complete solutions to the Cardy equation is equivalent to the search for $\mathbb{N}$ valued representations of the fusion algebra satisfying $n_{i}^{T}=n_{i^{*}}$.

This is the first important result of this paper, already presented succinctly in [32]. The fact that some solutions to the Cardy equation were associated with representations of the fusion algebra had been noticed before. In his seminal paper 10, Cardy considered the case of "diagonal theories" (for which $\mathcal{E}=\mathcal{I}$ ) and showed that the $n_{i}$ matrices were nothing other than the fusion matrices $N_{i}$, thus obtaining an alternative and more intuitive derivation of the Verlinde formula. In an antecedent work by Saleur and Bauer [9], other solutions had been obtained in non-diagonal theories, starting from their lattice realization, and the fact 
that these $n_{i}$ coefficients satisfied the fusion rules had been emphasized in [39]. More recently Pradisi, Sagnotti and Stanev [14, 15, 30] proposed a different argument to the same effect, where a notion of completeness of boundary conditions is also playing a crucial role.

Solutions such that all matrices $n_{i}$ may be written as $n_{i}=\left(n_{1}\right)_{i} \oplus\left(n_{2}\right)_{i}$ after the same suitable permutation of rows and columns can be called reducible. They describe sets of decoupled boundary conditions. We thus restrict our attention to irreducible sets of matrices.

\section{$2.3 \quad \mathrm{WZW} s l(2)$ theories}

For theories with the affine (current) algebra $\widehat{s l}(2)$ as a chiral algebra, the problem of classifying representations of the fusion algebra was solved long ago 39. The integrable highest weight representations of $\widehat{s l}(2)_{k}$ at level $k \in \mathbb{N}$ are labelled by an integer $1 \leq j \leq k+1$,

$S_{i j}=\sqrt{\frac{2}{k+2}} \sin \frac{i j \pi}{k+2}$ and the Cardy equation says that the generator $n_{2}=n_{2^{*}}$ has eigenvalues $\frac{S_{2 j}}{S_{1 j}}=2 \cos \frac{\pi j}{k+2}$. The only symmetric irreducible matrices with non-negative integer entries and eigenvalues less than 2 are the adjacency matrices of $A-D-E-T$ graphs 40 of Figure 2 (see also Table 1). The "tadpole" graphs are given by $T_{n}:=A_{2 n} / \mathbb{Z}_{2}$. Here the level $k$ is related to the Coxeter number by $g=k+2$. Only the $A-D-E$ solutions are retained as their spectrum matches the spectrum of $\widehat{s l}(2)$ theories, known by their modular invariant partition functions 41 . For a theory classified by a Dynkin diagram $G$ of $A-D$ - $E$ type, the set $\mathcal{E}$ is the set of Coxeter exponents of $G$ as in Table 1 . The matrices $n_{i}$ are then defined recursively by $n_{1}=I, n_{2}=G$ and by equation (2.22) which reduces here to $n_{i+1}=n_{2} n_{i}-n_{i-1}$, $i=2,3, \ldots, k$. They are the well known "fused adjacency matrices" or "intertwiners" $V_{i}$, studied in [39, 48] and whose properties are recalled in Appendix B. One verifies that all their entries are non-negative integers. This set of complete orthonormal solutions of the Cardy equation for $\widehat{s l}(2)$ theories is unique up to a relabelling of the states $|a\rangle$.

\subsection{Minimal $\operatorname{sl}(2)$ models}

The classification of $c<1$ minimal models in the bulk is given in terms of a pair of Dynkin diagrams $(A, G)$ where $G$ is of $A-D$-E type 41]. Let $h$ be the Coxeter number of $A_{h-1}$ and $g$ the Coxeter number of $G$ as given in Table 1 . Then the complete $A-D-E$ classification is

$$
\mathcal{M}(A, G)=\left\{\begin{array}{l}
\mathcal{M}\left(A_{h-1}, A_{g-1}\right) \\
\mathcal{M}\left(A_{h-1}, D_{(g+2) / 2}\right), \quad g \text { even } \\
\mathcal{M}\left(A_{h-1}, E_{6}\right) \\
\mathcal{M}\left(A_{h-1}, E_{7}\right) \\
\mathcal{M}\left(A_{h-1}, E_{8}\right)
\end{array}\right.
$$

with $h, g \geq 2$ and central charges given by

$$
c=1-\frac{6(h-g)^{2}}{h g} \text {. }
$$



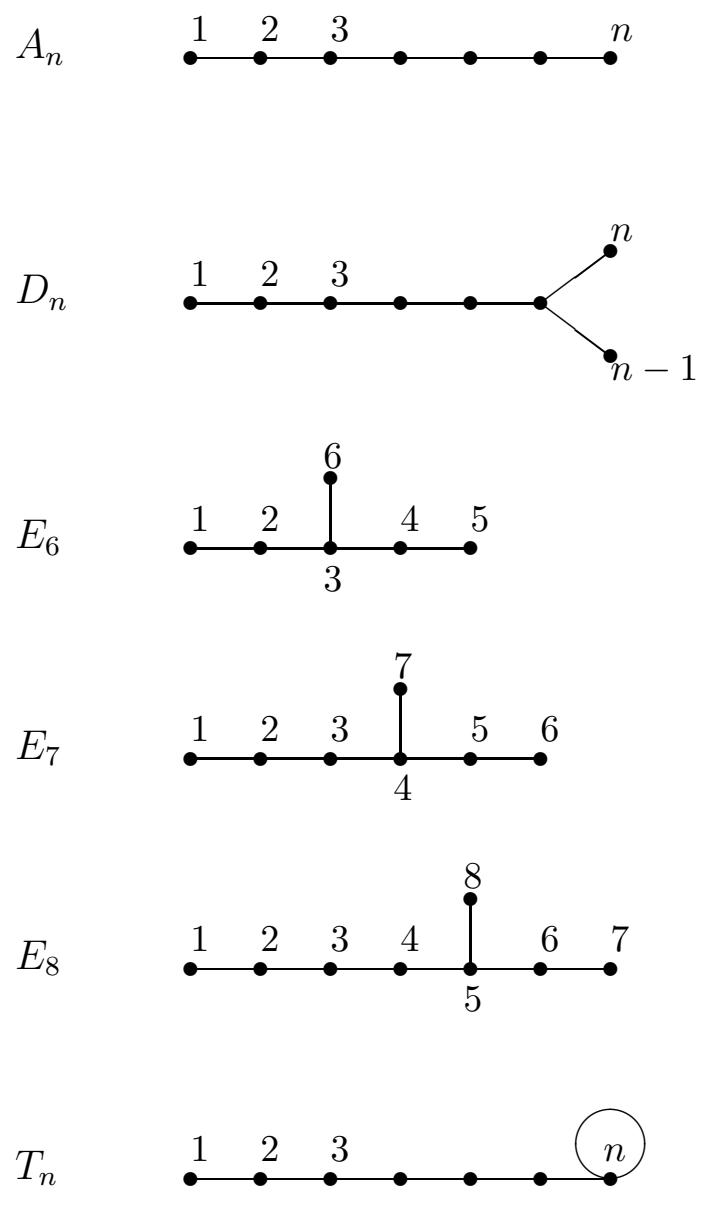

Figure 2: The $A-D-E-T$ graphs.

\begin{tabular}{ccc}
\hline$G$ & $g$ & $m \in \operatorname{Exp}(G)$ \\
\hline$A_{n}$ & $n+1$ & $1,2,3, \ldots, n$ \\
$D_{n}$ & $2 n-2$ & $1,3,5, \ldots, 2 n-3, n-1$ \\
$E_{6}$ & 12 & $1,4,5,7,8,11$ \\
$E_{7}$ & 18 & $1,5,7,9,11,13,17$ \\
$E_{8}$ & 30 & $1,7,11,13,17,19,23,29$ \\
$T_{n}$ & $2 n+1$ & $1,3,5, \ldots, 2 n-1$ \\
\hline
\end{tabular}

Table 1: The Coxeter number $g$ and Coxeter exponents $m$ of the $A-D-E-T$ graphs. 
We will use $\mathcal{M}(A, G)$ to denote these minimal theories. Since $g$ and $h$ must be coprime and $g$ is even for all non- $A$ cases, one may always assume, at the price of a possible interchange in the $(A, A)$ case, that $h$ is odd, $h=2 p+1$.

Some members of these series are identified as follows:

$$
\begin{array}{ll}
\mathcal{M}\left(A_{2}, A_{3}\right)=\text { critical Ising } & c=1 / 2 \\
\mathcal{M}\left(A_{4}, A_{3}\right)=\text { tricritical Ising } & c=7 / 10 \\
\mathcal{M}\left(A_{4}, D_{4}\right)=\text { critical 3-state Potts } & c=4 / 5 \\
\mathcal{M}\left(A_{6}, D_{4}\right)=\text { tricritical 3-state Potts } & c=6 / 7
\end{array}
$$

We will use $G$ to denote both the Dynkin diagram and its adjacency matrix. We use $r, r_{1}, r_{2}$ to denote nodes or exponents of $A_{h-1} ; s, s_{1}, s_{2}$ for the nodes (or exponents) of $A_{g-1}$; $a, a_{1}, a_{2}, b$ for the nodes of $G$. We refer the reader to Appendix B for more data on these matrices and their eigenvectors.

If $\operatorname{Exp}(G)$ denotes the set of exponents of $G$ (see Table 1), the modular invariant partition function of $\mathcal{M}\left(A_{h-1}, G\right)$ reads

$$
Z=\frac{1}{2} \sum_{r=1}^{h-1} \sum_{s \in \operatorname{Exp}(G)}\left|\chi_{r s}(q)\right|^{2}+\text { off-diagonal terms . }
$$

The factor $\frac{1}{2}$ removes the double counting due to the well-known identification of the $(r, s)$ and $(h-r, g-s)$ representations of the Virasoro algebra. The diagonal terms in $Z$, i.e. the left-right symmetric (highest weight) states in the spectrum are thus labelled by the set

$$
\mathcal{E}=\{j=(r, s) \equiv(h-r, g-s) ; 1 \leq r \leq h-1 ; s \in \operatorname{Exp}(G)\} .
$$

Each of the unitary minimal models $\mathcal{M}\left(A_{h-1}, G\right)$ with $g-h= \pm 1$ can be realized as the continuum scaling limit of an integrable two-dimensional lattice model at criticality, with heights living on the nodes of the graph $G$. In particular, the critical series with $g-h=1$ is associated with the A-D-E lattice models [43, 44] and the tricritical series with $g-h=-1$ is associated with the dilute lattice models [45, 46]. In the non-unitary cases the associated lattice models [47] possess negative Boltzmann weights. In the construction of the corresponding lattice models as well as in the description of boundary conditions, it turns out that the two diagrams of the pair $(A, G)$ do not play a symmetric role.

According to the discussion of the previous section, we have to study the fusion algebras of minimal models and their (integer-valued) representations. The Verlinde formula for the fusion coefficients makes use of the matrix $\mathcal{S}$ of modular transformations of characters

$$
\mathcal{S}_{r s, r^{\prime} s^{\prime}}=\sqrt{\frac{8}{g h}}(-1)^{(r+s)\left(r^{\prime}+s^{\prime}\right)} \sin \pi r r^{\prime} \frac{g-h}{h} \sin \pi s s^{\prime} \frac{g-h}{g}
$$

with the restriction $r, r^{\prime}$ odd (or any equivalent condition). The fusion coefficients are then found to be tensor products of those relative to the $\widehat{s l}(2)$ algebras of level $h-2$ and $g-2$, up to a symmetrization which accounts for the identification $(r, s) \equiv(h-r, g-s)$

$$
\mathcal{N}_{r s, r^{\prime} s^{\prime}}{ }^{r^{\prime \prime} s^{\prime \prime}}=N_{r r^{\prime}}{ }^{r^{\prime \prime}} N_{s s^{\prime}} s^{\prime \prime}+N_{r r^{\prime}}{ }^{h-r^{\prime \prime}} N_{s s^{\prime}}{ }^{g-s^{\prime \prime}} .
$$


This may be regarded as the regular representation of the fusion matrices $\mathcal{N}_{\text {rs }}$ of the Virasoro algebra of central charge (2.24). Our problem is to find the general non-negative integer valued representations of this algebra. One observes that $\mathcal{N}_{r s}=\mathcal{N}_{r 1} \mathcal{N}_{1 s}$ and that the algebra is thus generated by $\mathcal{N}_{21}$ and $\mathcal{N}_{12}$. Also, the eigenvalues of $\mathcal{N}_{12}$ and $\mathcal{N}_{21}$ are of the form

$$
\begin{aligned}
& \frac{\mathcal{S}_{12, r^{\prime} s^{\prime}}}{\mathcal{S}_{11, r^{\prime} s^{\prime}}}=(-1)^{r^{\prime}+s^{\prime}} 2 \cos \pi s^{\prime} \frac{g-h}{g}=(-1)^{r^{\prime}} 2 \cos \pi s^{\prime} \frac{h}{g}, \\
& \frac{\mathcal{S}_{21, r^{\prime} s^{\prime}}}{\mathcal{S}_{11, r^{\prime} s^{\prime}}}=(-1)^{s^{\prime}} 2 \cos \pi r^{\prime} \frac{g}{h} .
\end{aligned}
$$

with $1 \leq r^{\prime} \leq h-1,1 \leq s^{\prime} \leq g-1$ and again $\left(r^{\prime}, s^{\prime}\right) \equiv\left(h-r^{\prime}, g-s^{\prime}\right)$.

Turning now to a general (integer valued) representation $n_{r s}$ of the fusion algebra, it is still true that it is generated by $n_{12}$ and $n_{21}$. In addition, we want the spectrum of the $n_{r s}$ to be specified by the set of "exponents" $\mathcal{E}$ of $(2.23)$, that is $\left(r^{\prime}, s^{\prime}\right)$ in (2.30 2.31), with the eigenvalues labelled by $s^{\prime}$ appearing with some multiplicity in general. To remove the redundancy in the labelling of eigenvalues, we will usually take $r^{\prime}$ odd, $r^{\prime}=1,3, \cdots, h-2$, and $\left(s^{\prime}, \alpha\right) \in \operatorname{Exp}(G)$. In the sequel, we will drop this explicit notation for multiplicities. We know of course a solution to this problem, namely

$$
n_{r s}=N_{r} \otimes V_{s}+N_{h-r} \otimes V_{g-s}
$$

in terms of the fusion matrices $N$ of $\widehat{s l}(2)$ at level $h-2$ and of the intertwiners $V$ of type $G$ introduced in the previous subsection (see also Appendix B). More explicitly, this describes a solution to the Cardy equation between boundary states $\left(r_{1}, a\right)$ and $\left(r_{2}, b\right)$

$$
n_{r s ;\left(r_{1}, a\right)}{ }^{\left(r_{2}, b\right)}=N_{r r_{1}}^{r_{2}} V_{s a}{ }^{b}+N_{h-r r_{1}}^{r_{2}} V_{g-s a^{b}}
$$

with $1 \leq r, r_{1}, r_{2} \leq h-1=2 p, 1 \leq s \leq g-1$, and $a, b$ running over the nodes of the Dynkin $\operatorname{diagram} G$.

Because of the properties of the $N$ and $V$ matrices recalled in Appendix B, it is readily seen that

$$
n_{r s ;\left(r_{1}, a\right)}{ }^{\left(r_{2}, b\right)}=n_{r s ;\left(r_{1}, a\right)}{ }^{\left(h-r_{2}, \gamma(b)\right)}=n_{r s ;\left(h-r_{1}, \gamma(a)\right)}^{\left(r_{2}, b\right)}
$$

for an automorphism $\gamma$ acting on the nodes of the graph $G$ : this is the identity except for the $A, D_{\text {odd }}$ and $E_{6}$ cases, for which it is the natural $\mathbb{Z}_{2}$ symmetry of the diagram. We conclude that this solution describes boundary states of $\mathcal{M}\left(A_{h-1}, G\right)$ labelled by pairs $(r, a)$ of nodes of the $A_{h-1}$ and of the $G$ graph, with the identification

$$
(r, a) \equiv(h-r, \gamma(a))
$$

One checks that the number of independent boundary states $|(r, a)\rangle$ is

$$
\text { number of independent boundary states }=\frac{1}{2}(h-1) n
$$


with $n$ the number of nodes of $G$, or the number of its exponents. This number (2.36) coincides with the number of independent left-right symmetric highest weight states $|r, s\rangle \otimes$ $|r, s\rangle$ in the spectrum of the theory on a cylinder, i.e. with the cardinality of the set $\mathcal{E}$, as it should.

With such boundary states, the cylinder partition function reads

$$
Z_{\left(r_{1}, a\right) \mid\left(r_{2}, b\right)}=Z_{\left(r_{2}, b\right) \mid\left(r_{1}, a\right)}=Z_{\left(r_{1}, a\right) \mid\left(h-r_{2}, \gamma(b)\right)}=\sum_{r, s} \chi_{r s}(q) N_{r r_{1}}^{{ }^{r_{2}}} V_{s a}{ }^{b} .
$$

Here the sum runs over $1 \leq r \leq h-1,1 \leq s \leq g-1$.

Let us look more closely at (2.33). There exists a basis in which (2.33) takes a factorized form. Indeed one may use the identifications $(r, s) \equiv(h-r, g-s)$ and (2.35) to restrict $r, r_{1}, r_{2}$ to odd values (recall that $h=2 p+1$ is odd). Then $N_{h-r r_{1}}{ }^{r_{2}}=0$, the r.h.s. of (2.33) factorizes and the following expressions

$$
\Psi_{(r, a)}^{\left(r^{\prime \prime} s^{\prime \prime}\right)}=\sqrt{2} S_{r r^{\prime \prime}} \psi_{a}^{s^{\prime \prime}}, \quad r, r^{\prime \prime} \text { odd, } s^{\prime \prime} \in \operatorname{Exp}(G)
$$

written in terms of the modular matrix of $\widehat{s l}(2)$ at level $h-2$ and of eigenvectors $\psi$ of $G$, are readily seen to be eigenvectors of $n_{r s}$. Their eigenvalue is of the form $\mathcal{S}_{r s, r^{\prime} s^{\prime}} / \mathcal{S}_{11, r^{\prime} s^{\prime}}$ after some reshuffling $r^{\prime \prime}, s^{\prime \prime} \rightarrow r^{\prime}, s^{\prime}$.

One also shows (see Appendix C) that there exists a basis in which

$$
\begin{aligned}
& n_{12}=I_{p} \otimes G=\left(\begin{array}{llll}
G & & & \\
& G & & \\
& & \ddots & \\
& & & G
\end{array}\right), \\
& n_{21}=T_{p} \otimes \Gamma=\left(\begin{array}{cccc}
0 & \Gamma & & \\
\Gamma & 0 & \Gamma & \\
& \ddots & \ddots & \Gamma \\
& & \Gamma & \Gamma
\end{array}\right),
\end{aligned}
$$

in terms of the tadpole $T_{p}$ adjacency matrix and of $\Gamma$, the matrix that realizes the automorphism $\gamma: \Gamma_{a}^{b}=\delta_{a \gamma(b)}$.

Conversely, suppose we only know that the representation $n_{r s}$ has a spectrum specified by the set of exponents $\mathcal{E}$. The question is: are these spectral data sufficient to guarantee that the only $n_{r s}$ are of the form (2.33) in a certain basis? A proof of this fact is relegated to Appendix C. Notice that our discussion has assumed the classification of modular invariants to be known. It should be possible to extend it as in the case of WZW $\widehat{s l}(2)$ models and to classify the representations of the Verlinde algebra without this information. A few spurious cases involving tadpoles etc. would then have to be discarded.

To recapitulate, we have proved that the only representations of the fusion algebra of minimal models are given by (2.33). To our knowledge, this is the first proof of the uniqueness of these (complete orthonormal) boundary states of minimal models. 
Some physical intuition about the meaning of these boundary conditions may be helpful. For this we appeal to the lattice realization of the minimal model as a generalized height model on the graph $G$ (see [31]). A boundary condition of the type $(1, a)$ describes a fixed boundary condition, where the height of the model is fixed to value $a$ on the graph. The interpretation of the other label $r$ is less intuitive. The boundary condition $(r, a)$ is realized by attaching an $r$-times fused weight to height $a$.

The expression (2.37) for the cylinder partition function encompasses and generalizes cases that were already known:

- From the work of Saleur and Bauer [9] who discussed boundary conditions in lattice height models of $A-D-E$ type on a cylinder in which the heights on the boundaries are fixed to the values $a$ respectively $b$. They showed that in the continuum limit, the partition function reads

$$
Z_{b \mid a}=\sum_{s} V_{s a}^{b} \chi_{1 s}
$$

- From the work of Cardy [10] who showed how to construct new boundary conditions by fusion.

- From the work of Pasquier and Saleur [49], who interpreted the pair of relations

$$
\begin{aligned}
Z_{(1 b) \mid(1 a)}^{\left(A_{h-1}, G\right)} & =\sum \chi_{1 s} V_{s a}{ }^{b} \\
Z_{(1, s) \mid(1,1)}^{\left(A_{h-1}, A_{g-1}\right)} & =\chi_{1 s}
\end{aligned}
$$

as expressing the decomposition of the representation of the Temperley-Lieb algebra on the space of paths from $a$ to $b$ on graph $G$ onto the irreducible ones on the paths from 1 to $s$ on graph $A_{g-1}$, see point (ii) at the end of Appendix B.

\section{Examples}

Let us illustrate these expressions of boundary states by a few simple cases. In the Ising model (the $\left(A_{2}, A_{3}\right)$ minimal model), $h=3, G=A_{3}$, thus $n=3$ and there are $\frac{1}{2}(3-1) \times 3=3$ boundary states, generally denoted $[10]+,-$ and $f$. On the lattice, the first two describe fixed boundary conditions on the spin $\sigma=1$ or -1 respectively, while $f$ corresponds to free boundary conditions.

It is then instructive to consider two related examples, see also [22, 28]. The first is the $c=2 D_{4}$ solution of $\widehat{s l}(2)_{4}$ at level 4 , and the other is its cousin, the $c=4 / 5$ minimal (3-state Potts) model, already mentioned in Section 2.4 and labelled by the pair $\left(A_{4}, D_{4}\right)$. In the former case, we find four boundary states, labelled by 1 to 4 , that we attach to the nodes of the $D_{4}$ diagram. All these states satisfy the required boundary conditions. The set of exponents is $\mathcal{E}=\{1,3,3,5\}$. But this $D_{4} \widehat{s l}(2)$ model is also known to result from the conformal embedding of $\widehat{s l}(2)_{4}$ into $\widehat{s l}(3)_{1}$. Regarded as an $\widehat{s l}(3)$ theory, the model admits three boundary states satisfying the more restrictive $\widehat{s l}(3)$ conditions $\left(L_{n}-\bar{L}_{-n}\right)|a\rangle=$ $\left(J_{n}+\Omega \bar{J}_{-n}\right)|a\rangle=0$, where the choice of $\Omega$ corresponds to the diagonal set $(j, j)$. These 
three boundary states may be regarded as the three nodes of a triangular graph $\mathcal{A}^{(4)}$ (see Appendix D and Figure 10), or as the three extremal nodes of the $D_{4}$ diagram that have survived the additional $\widehat{s l}(3)$ constraint.

The discussion of the Potts model is quite parallel. From the minimal model standpoint, it is the $\left(A_{4}, D_{4}\right)$ model, $h=5, n=4$ and there are 8 boundary states [22, 28]:

$$
\begin{gathered}
A=(1,1)=(4,1), \quad B=(1,3)=(4,3), \quad C=(1,4)=(4,4) \\
B C=(2,1)=(3,1), \quad A C=(2,3)=(3,3), \quad A B=(2,4)=(3,4) \\
A B C=(1,2)=(4,2), \quad N=(2,2)=(3,2)
\end{gathered}
$$

On the lattice, the first three $A, B, C$ describe fixed boundary conditions where the "spin" takes at each site of the boundary one of the three possible values. The mixed boundary conditions $A B, B C, A C$ describe boundary conditions where the spin on the boundary can take on two values independently. The boundary conditions $A B C$ and $N$ are free boundary conditions but for $N$ the weights depend on whether adjacent spins are equal or not.

The model may also be regarded as the simplest $\mathcal{W}_{3}$ model. In that picture, one may impose more stringent boundary conditions. Only the six states denoted above $A, B, C$, $A B, B C, A C$ satisfy the additional condition $\left(W_{n}^{(3)}+\Omega \overline{W^{(3)}}-n\right)|a\rangle=0$. They correspond to the extremal nodes of the pair $\left(T_{2}, D_{4}\right)$ or, alternatively, to the nodes of the pair $\left(T_{2}, \mathcal{A}^{(4)}\right)$. As will be discussed in more detail in Section 3, the subset of these nodes, to be denoted $T$, can be identified in both examples with the representation labels of the corresponding extended chiral algebra. The matrix elements $\psi_{a}^{j}$ for $a \in T$ satisfy $\left[53\right.$ the relation $\frac{\psi_{a}^{j}}{\sqrt{S_{1 j}}}=$ $\frac{S_{a\{j\}}^{e x t}}{\sqrt{S_{\{1\}\{j\}}^{e x t}}}$, where $\{j\}$ denotes the orbit of the exponent $j$ with respect to the $\mathbb{Z}_{2}$ automorphism and $S_{a\{j\}}^{e x t}$ is the modular matrix of the extended theory. This relation implies that $|a\rangle=$ $\left.\sum_{\{j\}} \frac{S_{a\{j\}}^{e x t}}{\sqrt{S_{\{1\}\{j\}}^{\text {ext }}}} \sum_{j \in\{j\}}|j\rangle\right\rangle$, i.e., we can identify $\left.\left.\sum_{j \in\{j\}}|j\rangle\right\rangle=|\{j\}\rangle\right\rangle$ with an extended Ishibashi state. The missing boundary condition corresponds to a twisted boundary condition from the point of view of the extended algebra.

We conclude that, as expected, the number and nature of the boundary states reflect the precise conditions that they are supposed to satisfy.

\section{Graph Fusion Algebras}

According to the discussion of Section 2, given a certain chiral algebra $\mathcal{A}$, the sets of complete orthonormal boundary states of RCFTs consistent with this algebra are classified by representations of the Verlinde algebra of $\mathcal{A}$, on matrices $n_{i}$ with non-negative integral entries. Or stated differently: given a certain RCFT with a chiral algebra $\mathcal{A}$, the sets of complete orthonormal boundary states of this theory consistent with this algebra are classified by representations of the Verlinde algebra of $\mathcal{A}$, on matrices $n_{i}$ with non-negative integral entries, 
with eigenvalues specified by the diagonal part $\mathcal{E}$ of the spectrum. These matrices may thus be regarded as the adjacency matrices of a collection of $|\mathcal{I}|$ graphs. In practice, it is sufficient to look at the smaller number of matrices representing the generators of the fusion ring. For example, one matrix in the case of $\operatorname{sl}(2)$ considered above, or the $N-1$ matrices associated with the fundamental representations, in the case of $\operatorname{sl}(N)$.

The simplest case is given by the regular representation of the fusion algebra, when the matrices $n_{i}$ are the Verlinde matrices themselves, $n_{i}=N_{i}$. This is the case of socalled diagonal theories, when all representations of the set $\mathcal{I}$ appear once in the spectrum Spec $=\{(i, i) \mid i \in \mathcal{I}\}$. This may be regarded as the case of reference from several points of view: it was the first case analysed in detail [10]; the corresponding graphs are playing a central role; and finally in that case, Cardy was able to provide a physical argument explaining why the fusion matrices arise naturally. It is the purpose of this section to extend these considerations to more general solutions. We shall find that the role of the fusion matrices in the arguments of Cardy is now played by two sets of matrices. The first is the set of matrices $n_{i}$ that describe the coefficients of the cylinder partition function; the second is a new set of matrices $\hat{N}_{a}$, forming what is called the graph fusion algebra.

On the other hand, since we know that the cylinder partition functions, or equivalently the matrices $n_{i}$, contain some information about the bulk theory, through the knowledge of the diagonal spectrum $\mathcal{E}$, it is expected that this classification of boundary conditions should have some bearing on the classification of bulk theories, namely on the classification of torus partition functions and on bulk structure constants. Remarkably, this programme works even better than expected and the two classification problems seem to be essentially equivalent, at least for type I theories (see end of Section 1). This will be explained in Section 3.3 below.

\subsection{More on graphs and intertwiners}

Suppose we have found a solution to the Cardy equation, namely a set of $n \times n$ matrices (2.16), $\left(n_{i}\right)_{a}{ }^{b}, i=1, \cdots,|\mathcal{I}|, a, b=1, \cdots, n$. What was said in detail in Section 2 and in Appendix $B$ in the case of $\widehat{s l}(2)$ can be repeated here. As their entries are non-negative integers, these matrices may be regarded as adjacency matrices of a set of $|\mathcal{I}|$ graphs $G_{i}$, with $n=\left|G_{i}\right| \equiv|G|$ nodes. We shall refer collectively to these $|\mathcal{I}|$ graphs as "the graph $G$ ", whereas the basic solution provided by the $N$ 's themselves will be called "the $A$ graph", (borrowing the notation from the $s l(2)$ case). The eigenvalues of the matrices $n_{i}$ are specified by a set $\operatorname{Exp}(G)$ in the sense that they are of the form $S_{i j} / S_{1 j},(j, \alpha) \in \operatorname{Exp}(G)$. Moreover $\operatorname{Exp}(G)=\mathcal{E}$ if the RCFT is given and the diagonal spectrum $\mathcal{E}$ is known. But in general, the determination of the set $\operatorname{Exp}(G)$ is part of the problem. The fundamental relation $\sum_{b}\left(n_{i}\right)_{a}{ }^{b}\left(n_{j}\right)_{b}{ }^{c}=\sum_{k} N_{i j}{ }^{k}\left(n_{k}\right)_{a}{ }^{c}$ may be interpreted in two ways:

- Regarded as $|G| \times|G|$ matrices, the matrices $n_{i}$ form a representation of the fusion algebra (2.22). 
- Regarded as a $|\mathcal{I}| \times|G|$ rectangular matrix, each matrix $\tilde{n}_{a}$ for $a$ fixed, $\left(\tilde{n}_{a}\right)_{j}{ }^{b}:=n_{j a}{ }^{b}$ intertwines the representatives $N_{i}$ and $n_{i}$ in the two representations $N_{i} \tilde{n}_{a}=\tilde{n}_{a} n_{i}$, or more explicitly

$$
\sum_{k} N_{i j}{ }^{k} n_{k a}{ }^{c}=\sum_{b} n_{j a}{ }^{b} n_{i b}{ }^{c} .
$$

We shall thus occasionally refer to the matrices $n_{i}$ as "intertwiners".

The case of graphs and intertwiners pertaining to $\widehat{s l}(2)$ theories has been discussed at length in Section 2 and in Appendix B. In Appendix D, we outline the discussion of $\widehat{s l}(3)$. In that case, the fusion algebra is generated by two matrices $n_{(2,1)}$ and $n_{(1,2)}$ (labelled by the two fundamental (shifted) weights of $s l(3))$, and as these two representations are complex conjugate to one another, the matrices $n_{i}$ are related by transposition $n_{(2,1)}=n_{(1,2)}^{T}$. Then according to (2.22), $n_{i}$ is given by the same polynomial of $n_{(1,2)}$ and $n_{(2,1)}$ with integral coefficients as that representing $N_{i}$ in terms of $N_{(1,2)}$ and $N_{(2,1)}$. It is thus sufficient to list all possible graphs representing the matrix $n_{(2,1)}$, provided one checks that all $n_{i}$ have nonnegative integral entries. In contrast with the case of $\widehat{s l}(2)$, no complete solution is known for $\widehat{s l}(3)$. The current state of the art is presented in Appendix D with tables, figures and relevant comments.

\subsection{Graph fusion algebras}

To see what is playing the role of the fusion algebra in the argument of Cardy, we have to introduce the graph fusion algebra. The graph fusion algebra, as first discussed by Pasquier [50], is a fusion-like algebra attached to a connected graph $G$. Let $\psi_{a}^{j}$ be the common orthonormal eigenvectors of the adjacency matrices $G$ labelled by $j \in \operatorname{Exp}(G)$. In general, these eigenvectors can be complex. In the case of degenerate eigenvalues the associated eigenvectors need to be suitably chosen. We assume that the graph has a distinguished node labelled $1=1^{*}$ such that $\psi_{1}^{j}>0$, for all $j \in \operatorname{Exp}(G)$.

One then defines the numbers

$$
\hat{N}_{a b}^{c}=\sum_{j \in \operatorname{Exp}(G)} \frac{\psi_{a}^{j} \psi_{b}^{j}\left(\psi_{c}^{j}\right)^{*}}{\psi_{1}^{j}}
$$

and the matrices $\hat{N}_{a}$ with elements $\left(\hat{N}_{a}\right)_{b}{ }^{c}=\hat{N}_{a b}{ }^{c}$ satisfy $\hat{N}_{a b}{ }^{c}=\hat{N}_{a c^{*}} b^{*}$ and $N_{a}^{T}=N_{a^{*}}$. Because of orthonormality, $\hat{N}_{1}=I$. Since each matrix $\hat{N}_{a}$ has a single non-vanishing entry in the row labelled 1, namely $\left(\hat{N}_{a}\right)_{1}{ }^{b}=\left(\hat{N}_{1}\right)_{a}{ }^{b}=\delta_{a b}$, the matrices $\hat{N}_{a}$ are linearly independent. The $\hat{N}_{a b}{ }^{c}$ are the structure constants of the graph fusion algebra satisfied by the $\hat{N}$ matrices

$$
\hat{N}_{a} \hat{N}_{b}=\sum_{c} \hat{N}_{a b}^{c} \hat{N}_{c}
$$

which is an associative and commutative algebra. Of course, if the graph $G$ is of type $A$, this boils down to the ordinary Verlinde fusion algebra since the matrix $\psi$ of eigenvectors is nothing but the modular matrix $S$. 
Since the $\hat{N}_{a}$ and $n_{i}$ matrices have the same eigenvectors, it is easy to derive the matrix relation

$$
n_{i} \hat{N}_{a}=\sum_{b} n_{i a}^{b} \hat{N}_{b}
$$

In particular for $a=1, \hat{N}_{1}=I$, and all $n_{i}$ appear as linear combinations with non-negative integer coefficients of the $\hat{N}$ 's

$$
n_{i a}{ }^{b}=\sum_{c} n_{i 1}{ }^{c} \hat{N}_{c a}{ }^{b}
$$

Alternatively (3.4) may be used as a starting point to reconstruct the graph algebra, as explained in Section 3.5.

It should be stressed that the definition of a graph fusion algebra is not unique. In general, it depends on the choice of the distinguished node 1 and, when there are degenerate eigenvalues, also on the choice of the eigenvectors $\psi_{a}^{j}$. To view the graph fusion algebra as a proper fusion algebra we would like the structure constants $\hat{N}_{a b}{ }^{c}$ to be non-negative integers. But even the rationality of these numbers is not obvious and it is therefore surprising that, for appropriate choices of the $\psi$ 's and of node 1 and for most cases, they turn out to be integers of either sign. Among all the examples known to us in $\widehat{s l}(N)$ theories, $2 \leq N \leq 5$, it fails in only two cases: the graph called $\mathcal{E}_{5}^{(12)}$ in Figure 11 , for which there is no node 1 satisfying $1=1^{*}$, and whose $\hat{N}$ algebra involves fractions of denominator 4; and a graph in the $\widehat{s l}(4)_{4}$ theory, 53, in which half-integer $\hat{N}_{a b}{ }^{c}$ of either sign occur. Adopting (2.22), (3.4) in the framework of subfactors theory the latter example has been reinterpreted by $\mathrm{Xu}$ [55] by trading commutativity of the graph fusion algebra for integrality.

Finally the non-negativity of the $\hat{N}$ is only possible for certain graphs which we call proper fusion graphs. For example, for the $s l(2)$ theories, the $A-D-E$ graphs that admit a proper graph fusion algebra are

$$
\text { proper } A \text {-D-E graphs }=A_{n}, D_{2 q}, E_{6}, E_{8} \text {. }
$$

The choice of distinguished node for the $s l(2) A-D-E$ graphs is explained in Appendix B.

We note that the set of proper $s l(2)$ fusion graphs matches the modular invariant partition functions listed as "type I" at the end of Section 1. The situation is somewhat different for $\operatorname{sl}(3)$ graphs, for which we have to introduce a further distinction. In this case, some graphs with non-negative $\hat{N}$ 's are not associated with type I theories. We reserve the terminology "type I graph" for those graphs associated with type I theories (see Appendix D and Tables). Moreover, as is clear from the $\widehat{s l}(4)_{4}$ example above, some type I modular invariant partition functions are associated with graphs with non-integer and/or non-positive $\hat{N}_{a b}{ }^{c}$. In the following, we discard these exceptional cases and restrict ourselves to type I graphs that are associated with type I RCFTs. In general, the question of precisely which graphs admit type I fusion algebras should be related to the classification of type I RCFTs, and thus is a very interesting open question. 


\subsection{Fusion rules and block characters}

Given a solution to the Cardy equation, that is a set of partition functions

$$
Z_{a \mid b}(q)=\sum_{i \in \mathcal{I}} n_{i a}^{b} \chi_{i}(q)
$$

and the corresponding graphs, we assume as in Section 3.2 above that there exists a special node called 1 . We then introduce the combinations of characters (or "block characters")

$$
\hat{\chi}_{c}(q)=\sum_{i \in \mathcal{I}} \hat{n}_{i c} \chi_{i}(q)
$$

where

$$
\hat{n}_{i c}=n_{i 1}^{c}
$$

is referred to as the basic intertwiner. Thanks to (3.5), (3.7) may be rewritten as

$$
Z_{a \mid b}(q)=\sum_{c} \hat{N}_{c a}^{b} \hat{\chi}_{c}(q)
$$

where the coefficients are now given by the structure constants of the graph fusion algebra of $G$.

Equation (3.10) is a mathematical identity and as such is valid and consistent independent of the choice of the distinguished node and eigenvectors of $G$. Physically, however, the case where the $\hat{N}_{a b}^{c}$ are non-negative integers is the most interesting. In that case, following Cardy's discussion [10], it is suggested that $\hat{N}_{a b}^{c}$ gives the number of times that the propagating mode or representation $c$ appears in the strip or cylinder with boundary conditions $a$ and $b$. Thus if $G$ is a type I graph, i.e. if the structure constants $\hat{N}_{a b}{ }^{c}$ are non-negative integers, we have a possible interpretation: the nodes $a$ of the graph(s) $G$ under consideration label a class of representations of some extended chiral algebra. The blocks $\hat{\chi}_{a}$ are their characters, and the integer coefficients $\hat{N}_{a b}{ }^{c}$ are their fusion coefficients.

To probe this interpretation, let us see how it confronts the results "in the bulk", in particular how it is consistent with the form of the torus partition function. There, it has been observed already long ago that (for type I theories) the torus partition function (cf (1.5)) may be recast in the form

$$
Z_{\text {torus }}=\sum_{a \in T}\left|\hat{\chi}_{a}\right|^{2}
$$

i.e. as a diagonal sum over a subset $T$ of block characters. The subset $T$ corresponds to a subalgebra of the $\hat{N}$ algebra, in the sense that if $a, b \in T, \hat{N}_{a b}{ }^{c} \neq 0$ only if $c \in T$. This interpretation of $n_{i 1}{ }^{c}$ as a multiplicity of representation $i$ in the block $c$, that was first observed empirically [51], was subsequently derived in a variety of cases of type I $\operatorname{sl}(N)$ theories based either on conformal embeddings or on orbifolding [53, 54]. More recently, it appeared as an important ingredient in the investigation of the algebraic structure underlying these theories [55, 56, 57. 
The following interpretation is thus suggested. The nodes $a \in T$ label representations of the maximally extended algebra $\mathcal{A}^{\prime}$ of the RCFT (of type I) under consideration. The subalgebra of the $\hat{N}$ algebra is the conventional fusion algebra of this RCFT. The other nodes $a \notin T$ might label other "twisted" representations. The entire $\hat{N}$ algebra would describe the fusion of all, twisted and untwisted, representations of $\mathcal{A}^{\prime}$. This interpretation in terms of twisted representations seems corroborated by the fact that some $\hat{\chi}_{a}$ are known also to occur in partition functions on a torus in the presence of twisted boundary conditions. The fact that general boundary conditions on a cylinder also appeal to these representations was first observed in the Potts model in [22]. See for some work in this direction [58], and the more systematic developments [29] along the lines of [63]. The concept of twisted representations in other cases, like conformal embeddings, remains to be understood.

Having discussed the situation for type I theories and graphs, we return to RCFTs of type II. There the situation is more elusive. On the one hand, as discussed above, the boundary conditions on a cylinder are labelled by nodes of an improper graph $G$, and although we can still write an expression of the form (3.10), its physical interpretation is unclear. On the other hand, from (1.6), we know [73] that the torus partition function may be expressed in terms of block characters pertaining to a "parent" type I theory with graph $G^{\prime}$

$$
Z_{\mathrm{torus}}=\sum_{a \in T} \hat{\chi}_{a}(q)\left(\hat{\chi}_{\zeta(a)}(q)\right)^{*}
$$

where $T$ is once again a subset of the nodes of $G^{\prime}$ corresponding to a subalgebra of the $\hat{N}$ algebra, and $\zeta$ is an automorphism of that subalgebra $\hat{N}_{\zeta(a) \zeta(b)} \zeta(c)=\hat{N}_{a b}{ }^{c}$.

For example, the $\widehat{s l}(2)_{16}$ theory labelled by the Dynkin diagram $E_{7}$ is known to be related in that way to the $D_{10}$ theory. Their respective torus partition function read

$$
\begin{aligned}
Z_{\text {torus }}^{\left(D_{10}\right)} & =\left|\chi_{1}+\chi_{17}\right|^{2}+\left|\chi_{3}+\chi_{15}\right|^{2}+\left|\chi_{5}+\chi_{13}\right|^{2}+\left|\chi_{7}+\chi_{11}\right|^{2}+2\left|\chi_{9}\right|^{2} \\
& =\sum_{\substack{a=1,3,5,7,9,10 \\
a \in D_{10}}}\left|\sum_{i} \hat{n}_{i a}^{\left(D_{10}\right)} \chi_{i}\right|^{2} \\
Z_{\text {torus }}^{\left(E_{7}\right)} & =\left|\chi_{1}+\chi_{17}\right|^{2}+\left|\chi_{5}+\chi_{13}\right|^{2}+\left|\chi_{7}+\chi_{11}\right|^{2}+\left|\chi_{9}\right|^{2}+\left(\left(\chi_{3}+\chi_{15}\right) \chi_{9}^{*}+\text { c.c. }\right) \\
& =\sum_{\substack{a=1,3,5,7,9,10 \\
a \in D_{10}}}\left(\sum_{i} \hat{n}_{i a}^{\left(D_{10}\right)} \chi_{i}\right)\left(\sum_{i} \hat{n}_{i \zeta(a)}^{\left(D_{10}\right)} \chi_{i}\right)^{*}
\end{aligned}
$$

with $\zeta$ exchanging the two nodes 3 and 10 of the $D_{10}$ diagram.

It seems that the parent graph $G^{\prime}$ also plays a role for cylinder partition functions of type II theories. Indeed, to obtain cylinder partition functions expanded with non-negative coefficients in terms of block characters, we just have to expand in the block characters of $G^{\prime}$. Specifically, we find

$$
Z_{b \mid a}^{(G)}(q)=\sum_{c \in G^{\prime}} n_{c a}^{\left(G G^{\prime}\right) b} \hat{\chi}_{c}^{\left(G^{\prime}\right)}(q)
$$


where the $G-G^{\prime}$ intertwiners are given by

$$
n_{c a}^{\left(G G^{\prime}\right) b}=\sum_{m \in \operatorname{Exp}(G)} \frac{\psi_{c}^{\left(G^{\prime}\right) m}}{\psi_{1}^{\left(G^{\prime}\right)} m} \psi_{a}^{(G) m} \psi_{b}^{(G) m^{*}}
$$

These turn out to be non-negative integers $n_{c a}^{\left(G G^{\prime}\right) b} \geq 0$ and satisfy the $G^{\prime}$ graph algebra. Here it is assumed that the distinct exponents of $\operatorname{Exp}(G)$ are in $\operatorname{Exp}\left(G^{\prime}\right)$ and that the sum is over exponents of $G$ counting multiplicities. Moreover, if there is more than one eigenvector of $G^{\prime}$ corresponding to $m \in \operatorname{Exp}(G)$, then any of these eigenvectors can be matched with the given $m \in \operatorname{Exp}(G)$. The formula can be derived in the same way as our previous formulas. In particular, this formula applies for $G=E_{7}$ and $G^{\prime}=D_{10}$. In this case there is an ambiguity as to which $D_{10}$ eigenvector is taken for $m=9$ but in fact one can take either. The matrices are changed by the $Z_{2}$ symmetry but the cylinder partition functions agree. The formula also holds for the type II $\operatorname{sl}(3)$ theories $G=\mathcal{E}_{2}^{(12)}, \mathcal{E}_{4}^{(12)}$ and $\mathcal{E}_{5}^{(12)}$ and $G^{\prime}=\mathcal{D}^{(12)}$.

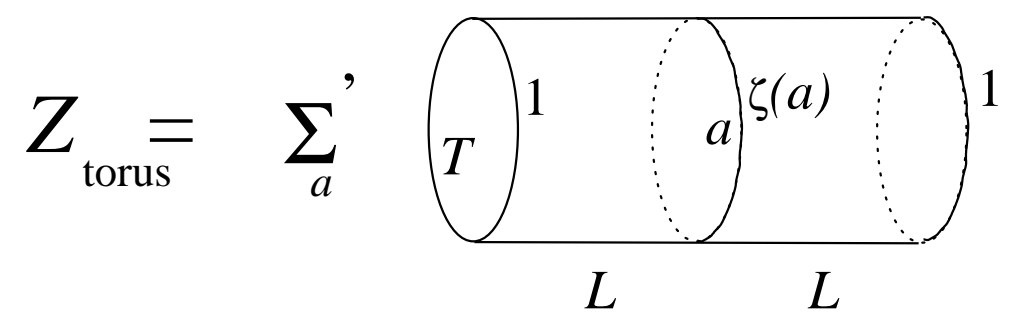

Figure 3: The torus partition function reconstructed from two cylinder partition functions.

Putting everything together, we finally observe that in general for a "rectangular" torus with two periods $2 L$ and $i T$, made by pasting together two cylinders, (see Figure 3),

$$
Z_{\text {torus }}^{(G)}=\sum_{a \in T} Z_{a \mid 1}^{\left(G^{\prime}\right)} Z_{\zeta(a) \mid 1}^{\left(G^{\prime}\right)}
$$

i.e. the partition function may be obtained as the sum over a special set of boundary conditions of cylinder partition functions. This expression is of course deeply rooted in all the connections between bulk and boundary theories, open and closed strings, etc, but still we find its simplicity intriguing.

\subsection{Examples}

More examples can be given to the previous general scheme.

- $\hat{s l}(N)$ : the classification of the representations of the fusion algebra of $\widehat{s l}(N)_{k}$ is a well posed problem on which we have only partial results. In particular, classes of graphs pertaining to $\widehat{s l}(3)$ as well as some cases for higher $N$ have been expounded from various standpoints in [39, 51, 52, 53, 54, 59], (see Appendix D). In all known cases, the previous discussion may be repeated: intertwiners, type I graphs, and other concepts introduced above, still apply. We refer the reader to the above references. 
- The case of $\hat{s l}(N)_{1}$ may be described in detail. The representations of $\widehat{s l}(N)_{1}$ are labelled by an integer $0 \leq i \leq N-1$ (we depart here from our previous convention, with $i=0$ denoting the identity). The fusion rules are isomorphic to the addition of integers modulo $N$, and the algebra defined by $N_{i} N_{j}=N_{i+j \bmod N}$ is thus generated by $N_{1}, N_{j}=\left(N_{1}\right)^{j}$. The eigenvalues of $N_{j}$ are $\exp 2 i \pi j l / N$. The regular representation is provided by $N \times N$ matrices, generated by $\left(N_{1}\right)_{i}{ }^{j}=\delta_{j, i+1 \bmod N}$. All the previous eigenvalues are reached once and we may thus say that the system has "exponents" $l=0, \cdots, N-1(\bmod N)$. In general, a representation $\left\{n_{i}\right\}$ of the fusion algebra is associated with each divisor $q$ of $N=p . q$, including $q=1$ and $q=N$ : $q$ denotes the order of the matrix $n_{1}$ which is $q \times q$ dimensional and such that $\left(n_{1}\right)_{a}{ }^{b}=\delta_{b-a, 1 \bmod q}$, for a labelling of the nodes $a, b=1, \cdots, q$. (This exhausts all integer-valued representations of the algebra. Indeed the conditions that $n_{1}^{T}=n_{N-1}$ and $n_{1} n_{N-1}=I$ imply that the only entries of $n_{1}$ are 0 and 1 , and that $n_{1}$ is a permutation matrix. Being of order $q$ and indecomposable, $n_{1}$ is a matrix of a cycle of length $q$. Q.E.D.) Obviously the matrices $n_{i}=\left(n_{1}\right)^{i}$ are all integer-valued, $n_{0}=I$, and $n_{i}^{T}=n_{i^{*}}=n_{N-i}$. The graph of adjacency matrix $n_{1}$ is an oriented $q$-gon. In that case, we may say that the $q$ exponents are $0, p, \cdots, p(q-1)$.

This census of representations of the $\widehat{s l}(N)_{1}$ fusion algebra matches almost perfectly that of modular invariant partition functions carried out by Itzykson [60] and Degiovanni [61. We recall that according to these authors, a different modular invariant is associated with each divisor of $n$, where $n=N$ if $N$ is odd and $n=N / 2$ if it is even. Thus, only the case $N$ even, $q=1$ has to be discarded in our list of representations of the fusion algebra, as it does not correspond to a modular invariant.

\subsection{More on graph algebras}

In Section 3.2, we have introduced the matrices $\hat{N}$ by (3.2) and derived (3.4). Instead of looking at the graph as a collection of points we can look at it as a collection of matrices $\hat{N}$, providing a basis of a commutative, associative algebra with identity, and an action of the intertwiners $n_{i}$ given by (3.4), that is, we take (3.4) as a starting point. Given the graph $G$, in particular the coefficients $n_{i 1}{ }^{c}$, it is possible in many cases to invert (3.5) and solve for $\hat{N}_{a}$ as linear combinations with integral coefficients of the intertwiners $n_{i}$, or equivalently, as polynomials of the fundamental adjacency matrices. Similarly the relation (3.4) written in terms of the eigenvalues $\gamma_{j}(i)=\frac{S_{j i}}{S_{1 i}}, \hat{\gamma}_{a}(i)=\frac{\psi_{a}^{i}}{\psi_{1}^{i}}$

$$
\gamma_{j}(i) \hat{\gamma}_{a}(i)=\sum_{b} n_{j a}{ }^{b} \hat{\gamma}_{b}(i), \quad i \in \mathcal{E}, j \in \mathcal{I}
$$

is a recursive relation determining (the rows of) the eigenvector matrix $\psi_{a}^{i}$. In general, typically in the presence of degenerate eigenvalues, the matrix $\psi_{a}^{i}$ is not determined uniquely, or alternatively, (3.5) cannot be inverted for all $a \in \mathcal{V}$. For the type I cases, however, as explained above, there exists an extended fusion algebra isomorphic to a subalgebra of the 
graph algebra, so that the extended fusion matrices $N_{B_{i}}^{e x t}$ can be identified with a subset $\hat{N}_{a}$ with the nodes $a \in T \subset \mathcal{V}$ [51. In most of these type I cases one can solve for all $\hat{N}_{a}$ in terms of the $n_{i}$ 's and $N_{B_{i}}^{e x t}$, or alternatively express $\psi_{a}^{j}$ in terms of the modular matrices $S_{i j}, S_{B_{s} B_{l}}^{e x t}$. A particularly simple subclass of Type I for which one can go quite far in the programme of reconstructing the graph $\mathrm{G}$ and all the related structures is presented by the orbifold theories, in particular the ones associated with groups generated by simple currents. In our approach they can be described by graphs obtained by 'orbifolding' the fundamental graphs of the initial diagonal theory, the simplest example being provided by the WZW $\operatorname{sl}(2)$ $D_{2 l}$ series obtained by "orbifolding" the Dynkin diagram $A_{4 l-3}$ over the $\mathbb{Z}_{2}$ group generated by the automorphism $\gamma$. In these cases as well as in their $\operatorname{sl}(N)$ generalisations defined in [62 involving the group $\mathbb{Z}_{N}$, one can algorithmically construct the eigenvector matrix, see Appendix B for an illustration in the simplest $N=2$ case. In a different approach, using tools similar to the original orbifold treatment of [63], an elegant general formula for the eigenvector matrix was derived recently in [29]. It should be noted that the same graph (orbi)folding procedure leads also to type II graphs, e.g., the $\operatorname{sl}(2) D_{\text {odd }}$ series, or their $\operatorname{sl}(3)$ generalisations for $k \neq 0 \bmod 3$, see Appendix D.

We have assumed up to now in this discussion that the graphs are already known. On the other hand the relations (3.4), (2.22) can be taken as the starting point for finding new graphs, typically "exceptional" graphs not covered by the previous orbifold constructions. Since any graph in the vicinity of the identity resembles the original "diagonal" $(A)$ graph, one can first try to identify $n_{i}$ 's for which the r.h.s. of (3.5) reduces to one term, i.e., $n_{i 1}{ }^{a}=\delta_{a a_{i}}$ and hence one can identify $n_{i}=\hat{N}_{a_{i}}$. According to (3.18) this also determines $\psi_{a_{i}}^{j}$ by $\gamma_{i}(j)$ once $\psi_{1}^{j}$ is known. This is a problem which is reduced to the computation of some Verlinde fusion multiplicities. Indeed let us take the first matrix element $a=b=1$ of the matrix relation $(2.22$ ) we have

$$
\sum_{c} n_{i 1}{ }^{c} n_{j 1}{ }^{c}=\sum_{l} N_{l i}^{j} n_{l 1}^{1}=\sum_{l \in \rho} N_{l i}^{j}
$$

where in the last sum $\rho=\left\{l \in \mathcal{I} \mid n_{l 1}{ }^{1} \neq 0\right\}$ and $l$ is counted $n_{l 1}{ }^{1}$ times. Let us assume first that in (3.19) $i=j$. Whenever the r.h.s. of (3.19) is equal to 1 , since by definition $n_{i 1}{ }^{a}$ are integers, the 1.h.s. summation reduces to one term, i.e., we recover $n_{i 1}{ }^{a}=\delta_{a a_{i}}$. Furthermore plugging this into the l.h.s. of (3.19) taken for $j \neq i$ we recover $n_{j 1} a_{i}$ as being given by the sum of Verlinde fusion multiplicities in the r.h.s. of (3.19), i.e., we determine the multiplicity with which $N_{a_{i}}$ appears in $n_{j}$, see (3.5). Similarly, a value 2 or 3 for the r.h.s. of (3.19) with $i=j$ would lead to 2 , respectively 3 terms in (3.5), while 4 could be interpreted either as leading to 4 terms with multiplicity one, or 1 term with multiplicity two, i.e., $n_{i 1}{ }^{a}=2 \delta_{a a_{i}}$. What we only need in order to check all these possibilities is to know the content of the set $\rho$, i.e., $n_{i 1}{ }^{1}$. This data is provided in type I theories for all of which $\rho$ encodes the content of the identity representation of the extended algebra. More generally, $n_{i 1}{ }^{a}=\operatorname{mult}_{B_{a}}(i)$, identifying $a$ with a representation $B_{a}$ of the extended algebra. The relation (3.19) and its consequences just described are the first steps in a consistent 
algorithmic procedure proposed by $\mathrm{Xu}$ [55] in the abstract framework of subfactors theory (see also 56, 57 for further developments). In particular, the subset of $n_{i}$ which can be identified with some $\hat{N}_{a_{i}}$ are related to "irreducible" sectors with the sum in the l.h.s. of (3.19) interpreted as a scalar product $\left(a_{i}, a_{j}\right)$. The algorithm reduces systematically the determination of $n_{i}, \hat{N}_{a}$ in type I cases to data provided by the Verlinde fusion matrices $N$ and $N^{e x t}$. All graphs previously found in [39, 51, 53] were recovered in [55] by this method and a new example corresponding to the $\widehat{s l}(4)_{6}$ modular invariant was found in [54.

Finally let us point out that to some extent this algorithm for finding new solutions of the equations (3.4) and (2.22), i.e., new graphs, can be applied to type II cases where we do not know a priori $n_{i 1}{ }^{1}$, i.e., the set $\rho$. One can start with some trial set and compute $\sum_{\alpha}\left(\psi_{1}^{(j, \alpha)}\right)^{2}=S_{1 j} \sum_{l \in \rho} S_{l j}$. A first consistency check is that $\sum_{j \in \mathcal{E}}\left(\psi_{1}^{j}\right)^{2}=1$. Then one can proceed as in type I. For example the $E_{7}$ Dynkin diagram may be reconstructed using $\rho=\{1,9,17\}$ ? ${ }^{3}$. Different (consistent) choices of the set $\rho$ might lead to the same graph, reflecting the possibility of different choices of the identity node.

In some simple cases it is possible to recover a complete set of boundary conditions by applying formula (3.4) to a known subset in such a way that only one term appears in the sum in the r.h.s. In terms of the equivalent formula (3.18) for the eigenvalues we obtain a new solution $\psi_{b}^{i}, b=b(a, j)$, by "fusing" a given one $\psi_{a}^{i}$ with the Verlinde eigenvalue $\gamma_{j}(i)$. This seems to be the idea of the so called "Affleck fusion conjecture" [17, which clearly has a restricted application, with the general formulae (3.4), (3.18) being the correct substitute for it.

Another approach to constructing Type I graphs was discussed in [53 and used to find new solutions for higher rank cases. It is based on the use of a relation for the structure constants of the Pasquier algebra, the dual of the graph algebra, with structure constants labelled by elements in the $\operatorname{set} \operatorname{Exp}(\mathrm{G})$ and given by a formula analogous to (3.2), however with the summation running over the nodes of the graph; this algebra will be discussed further in section 4.4.1 below.

\section{Bulk and Boundary Operator Algebras}

In this section we investigate the algebras of fields in the presence of boundaries and the equations for their structure constants resulting from duality constraints. Our discussion parallels that of Cardy and Lewellen [11], but generalises it in two respects: to higher rank and to non-diagonal theories. This results in additional multiplicities associated with the more general representations of the Verlinde algebra (2.22). Our presentation makes use of concepts used by Moore and Seiberg for bulk RCFTs and extends them appropriately for this new setting. This leads to a richer structure in the equations and the appearance of

\footnotetext{
${ }^{3}$ Note added in proof: this was independently discussed in the recent paper [86], see also [73]. The paper 86 provides a systematic approach in the framework of the subfactors theory to both types of modular invariants.
} 
a triplet of algebras $\left(n_{i}, \hat{N}_{a}, M_{j}\right)$. Separately these algebras have appeared before but the inter-relation between these algebras has not been shown in this context.

\subsection{Ground state degeneracies}

As stressed by Affleck and Ludwig [16, the logarithm of the partition function, in the limit $L / T \rightarrow \infty$, contains not only the universal term proportional to $L$ and to the central charge (in unitary theories), but also an $L$ independent term, interpreted as a boundary condition dependent "ground state degeneracy" $\ln g_{a} g_{b}$. Indeed in that limit

$$
\ln Z_{b \mid a} \sim \frac{c}{24} 4 \pi \frac{L}{T}+\ln \psi_{a}^{1}+\ln \psi_{b}^{1}-\ln S_{11}
$$

where as before we denote by 1 the representation of conformal weight 0 , corresponding to the identity operator. We therefore identify

$$
g_{a}=\frac{\psi_{a}^{1}}{\sqrt{S_{11}}} .
$$

Thus in unitary minimal models, using (2.38) and (2.28), we have the following expression for the boundary states

$$
\left.|(r, a)\rangle=\sum_{\substack{r^{\prime}, s^{\prime} \\ r^{\prime} \text { odd }, s^{\prime} \in \operatorname{Exp}(G)}} 2^{\frac{1}{4}} \frac{S_{r r^{\prime}}^{(h)} \psi_{a}^{s^{\prime}}}{\sqrt{S_{1 r^{\prime}}^{(h)} S_{1 s^{\prime}}^{(g)}}}\left|r^{\prime}, s^{\prime}\right\rangle\right\rangle
$$

and their $g$ factor

$$
g_{(r, a)}=\frac{\langle\langle 1,1 \mid(r, a)\rangle}{\sqrt{S_{11}^{(h)} S_{11}^{(g)}}}=2^{\frac{1}{4}} \frac{S_{r 1}^{(h)} \psi_{a}^{1}}{\sqrt{S_{11}^{(h)} S_{11}^{(g)}}},
$$

in terms of the modular matrices $S^{(h)}$ and $S^{(g)}$ of $\widehat{s l}(2)$ at levels $h-2$ and $g-2,|g-h|=1$. For example, for the critical 3-state Potts model, we obtain

$$
g_{A}=\left(\frac{5-\sqrt{5}}{30}\right)^{\frac{1}{4}}=0.550936 \quad\left(g_{A}: g_{A B}: g_{A B C}: g_{N}\right)=\left(1: \frac{1+\sqrt{5}}{2}: \sqrt{3}: \frac{1+\sqrt{5}}{2} \sqrt{3}\right)
$$

in agreement with [22]. As a particular case of (4.4), the ratio $g_{(r, a)} / g_{(1, a)}$ equals $S_{r 1}^{(h)} / S_{11}^{(h)}$, in agreement with (A.3) of [22] and the fact that one obtains the boundary state $|(r, a)\rangle$ by fusion (in the sense of Cardy) of boundary states $(1, a)$ and $(r, 1)$.

In non-unitary cases, these expressions have to be slightly amended. If $j_{0}$ denotes the representation of smallest conformal weight $h_{j_{0}}<0$ and assumed to belong to $\mathcal{E}$, then

$$
\ln Z_{b \mid a} \sim \frac{c_{\mathrm{eff}}}{24} 4 \pi \frac{L}{T}+\ln \psi_{a}^{j_{0}}+\ln \psi_{b}^{j_{0}}-\ln S_{1 j_{0}}
$$

with $c_{\text {eff }}:=c-24 h_{j_{0}}$. Also

$$
g_{a}=\frac{\psi_{a}^{1}}{\sqrt{S_{1 j_{0}}}}
$$


For simplicity of notation in most of what follows we shall restrict to unitary theories. Denoting $g_{a}=\langle\mathbf{1}\rangle_{a}$ from now on,

$$
\lim _{L / T \rightarrow \infty} Z_{b \mid a} e^{-\frac{\pi c}{6} \frac{L}{T}} / g_{b}=\langle\mathbf{1}\rangle_{a} .
$$

One can consider furthermore the partition function with some field insertions at the same limit [11], 25], [26]; we shall normalise them similarly so that only a dependence on $g_{a}$ is retained, i.e., $\langle\mathbf{1}\rangle_{a}$ will coincide with the 1-point function of the identity operator in this limit.

\subsection{Bulk and boundary fields, OPE}

\subsubsection{Boundary fields}

According to Cardy [10], boundary conditions can be interpreted as created by the insertion of fields ${ }^{b} \Psi_{j, \beta}^{a}(x)$ living on the boundary, Im $z=0, x=\operatorname{Re} z$ of the upper half-plane $z \in H_{+}$. Here $j \in \mathcal{I}, a, b \in \mathcal{V}$, and $\beta$ accounts for the multiplicity $n_{j a}{ }^{b}$ of such fields, to be called "of type" $\left(\begin{array}{l}b \\ j a\end{array}\right)$. Thus $\beta$ can be interpreted as a "coupling" index $\beta=1,2 \ldots, n_{j a}{ }^{b}$ and the boundary fields as kind of "chiral vertex operators" (CVO) associated with a second type of couplings $\left(\begin{array}{l}b \\ j a\end{array}\right)_{\beta}, a, b \in \mathcal{V}, j \in \mathcal{I}$. This is a formal analogy since the boundary states $|a\rangle,|b\rangle$ labelled by $a, b$ are superpositions of Ishibashi states. The multiplicity index $\beta$ is traditionally omitted, but it should be stressed that even in the $s l(2)$ case, in all but the diagonal cases $(\mathcal{E}=\mathcal{I})$, there are always some non-trivial multiplicities $n_{j a}{ }^{b}>1$, so most of the time we shall retain this index. Since $n_{1 a}{ }^{b}=\delta_{a b}$ the index associated with the coupling $\left(\begin{array}{l}b \\ 1 b\end{array}\right)$ takes only one value and will be denoted $\beta=\mathbf{1}_{b}$, or just $\mathbf{1}$, or, altogether omitted. On the other hand in all non-diagonal cases there is a non-trivial subset $\left\{{ }^{1} \Psi_{j}^{1}, j \in \rho\right\}$ of boundary fields, with $1 \in \rho \subset \mathcal{I}$, where the set $\rho$ has been introduced in Section 3.5.

To make contact with Section 2.2, consider the finite strip $w=\frac{L}{\pi} \log z$ equipped with the Hamiltonian $H_{a b}=\frac{\pi}{L}\left(L_{0}^{(H)}-\frac{c}{24}\right)$. The space of states is generated by all the descendent states created for fixed $a, b$ from the (properly normalised) vacuum state ${ }^{b} \Psi_{j, \beta}^{a}(0)|0\rangle$ by the modes of the Virasoro algebra generating the real analytic conformal transformations. This includes besides the sum over $j \in \mathcal{I}$ a summation over the multiplicity $n_{j a}{ }^{b}$ of these states for fixed $a, b, j$, i.e., we can think of the Vir representation spaces $\mathcal{V}_{j, \beta}$ as being labelled by pairs $(j, \beta)$. The "dual vacuum state" is defined by a boundary field placed at infinity $\sum_{\beta^{\prime}} \lim _{x \rightarrow \infty} c_{a, b, j ; \beta, \beta^{\prime}} x^{2 \triangle_{j}}\left\langle\left. 0\right|^{a} \Psi_{j^{*}, \beta^{\prime}}^{b}(x)\right.$ where $c_{a, b, j ; \beta, \beta^{\prime}}$ is a normalisation constant and $\beta^{\prime}=1, \cdots, n_{j^{*} b}{ }^{a}$ is an index of type $\left(\begin{array}{c}a \\ j^{*} b\end{array}\right)$. Accordingly the trace of the operator $e^{-T H_{a b}}$ computed imposing the periodicity $w \sim w+T$ in time direction can be written as a sum over $(j, \beta)$ of characters $\chi_{j, \beta}$, with the summation over $\beta$ leading to (2.15), with $a$ and $b$ exchanged.

Boundary fields ${ }^{b} \Psi_{j, \beta}^{a}(x)$ appear as ordinary fields $\Psi_{j}(x)$ decorated by a pair of indices $a, b$ according to some rules. In the limit $L / T \rightarrow \infty$ their 1-, 2- and 3-point functions 
are given by the corresponding invariants of $\Psi_{j}$ with respect to $\operatorname{sl}(2, \mathbb{R})$ with normalisation coefficients depending on $a, b$. As for the ordinary fields the 1-point function is non-zero only for the identity operator

$$
\left\langle\left. 0\right|^{b} \Psi_{j, \alpha}^{a}(x) \mid 0\right\rangle=\delta_{j 1} \delta_{b a} \delta_{\alpha \mathbf{1}_{a}}\langle\mathbf{1}\rangle_{a}
$$

with the restriction on $a, b$ coming from $n_{1 a}{ }^{b}=\delta_{a b}$. As for the ordinary CVO the product ${ }^{b} \Psi_{i, \alpha_{1}}^{c}{ }^{d} \Psi_{j, \alpha_{2}}^{a}$ of two boundary fields is defined only for coinciding $c=d$. Similarly the initial and the final indices in a vacuum expectation value of a product of boundary fields, are restricted to coincide (due to the periodicity in the strip time direction, the boundary halfline being effectively closed) but in distinction with the ordinary CVO they can be arbitrary and not just equal to the identity 1 . The 2- and 3-point functions read

$$
\begin{gathered}
\left\langle\left. 0\right|^{a} \Psi_{j, \alpha_{1}}^{b}\left(x_{1}\right)^{b} \Psi_{i, \alpha_{2}}^{c}\left(x_{2}\right) \mid 0\right\rangle=\delta_{j i^{*}} \delta_{a c} \frac{C_{i^{*} i ; \alpha_{1} \alpha_{2}}^{a b}}{\left|x_{12}\right|^{2 \triangle_{j}}}, \quad x_{1} \neq x_{2}, \\
\left\langle\left. 0\right|^{a} \Psi_{i, \alpha_{1}}^{b}\left(x_{1}\right)^{b} \Psi_{j, \alpha_{2}}^{c}\left(x_{2}\right)^{c} \Psi_{k, \alpha_{3}}^{d}\left(x_{3}\right) \mid 0\right\rangle_{t}=\delta_{a d} \frac{C_{i j k ; \alpha_{1} \alpha_{2} \alpha_{3} ; t}^{a b c}}{\left|x_{12}\right|^{\triangle_{i j}^{k}}\left|x_{23}\right|^{\triangle_{j k}^{i}}\left|x_{31}\right|^{\triangle_{k i}^{j}}}, \quad x_{1} \neq x_{2} \neq x_{3} \neq x_{1},
\end{gathered}
$$

where $\Delta_{i j}^{k}=\Delta_{i}+\Delta_{j}-\Delta_{k}$ and $x_{i j}=x_{i}-x_{j}$.

The functions $(4.9),(4.10)$ are invariant with respect to $S L(2, \mathbb{R})$, with representations denoted by a pair $(\delta, \varepsilon= \pm)$, see 42 ; here we choose $\varepsilon=1$ corresponding to taking the modulus of the multiplier of the $S L(2, \mathbb{R})$ transformations and the expressions in (4.9), (4.10) imply trivial monodromy of the boundary field correlators. In the $\widehat{s l}(n)_{k}$ WZW models the fields carry an additional tensor index, or, in a functional realisation, depend on an additional (multi)variable $X$ accounting for the representations of the "isospin" $\operatorname{sl}(n, \mathbb{R})$ algebra and the $n$-point functions involve also $n$-point invariants with respect to this algebra. For example, in the $\hat{s l}(2)_{k}$ WZW case the fields $\Psi_{j}(x, X)$ can be described in terms of a pair of real variables [68, the coefficients in the polynomial expansion with respect to $X$ representing the horizontal algebra descendants. In this case the isospin labels are $2 j=0,1, \ldots, k$, and the 2- and 3-point invariant correlators contain additional factors $X_{i l}^{2 j}$ along with any $x_{i l}^{-2 \triangle_{j}}$. For simplicity we adapt the notation for the minimal $W_{n}$ models rather than their WZW counterparts, omitting the explicit indication of the "isospin" variables and the corresponding invariants. To keep track of the various possible three-point invariants, we shall retain the multiplicity index $t$ as in (4.10).

Up to the normalisation constant and up to phases, (4.10) is the 3-point invariant function $\left\langle 0\left|\phi_{i, \mathbf{1}_{i}^{\prime}} \phi_{j, t} \phi_{k, \mathbf{1}_{k}}\right| 0\right\rangle$ of the ordinary CVO $\phi_{j, t}(x)$. Here $t$ is a coupling index of type $\left(\begin{array}{c}i^{*} \\ j k\end{array}\right), t=1,2, \ldots, N_{j k}{ }^{i^{*}}$. Two kinds of permutations act on these couplings, see [33]: $\sigma_{23}$ $\left(\begin{array}{c}p \\ i j\end{array}\right) \rightarrow\left(\begin{array}{c}p \\ j i\end{array}\right)$, and $\sigma_{13}:\left(\begin{array}{c}p \\ i j\end{array}\right) \rightarrow\left(\begin{array}{l}j^{*} \\ i p^{*}\end{array}\right)$. For simplicity in the sequel we denote the one value indices indicating couplings with one label of type $\mathcal{I}$ set to 1 , like $\sigma_{23}\left(\mathbf{1}_{i}\right)$, $\mathbf{1}_{i}^{\prime}$, or, $\mathbf{1}_{a}$ (corresponding to couplings of type $\left(\begin{array}{c}i \\ i 1\end{array}\right),\left(\begin{array}{l}1 \\ i i^{*}\end{array}\right)$, or, $\left(\begin{array}{l}a \\ 1 a\end{array}\right)$ resp.), simply by 1 . 
Motivated by the form of the 3-point function, the operator product expansion (OPE) of (primary) boundary fields ${ }^{b} \Psi_{i, \alpha}^{c}(x)$ is defined according to

$$
\begin{aligned}
{ }^{b} \Psi_{i, \alpha_{1}}^{c}\left(x_{1}\right){ }^{c} \Psi_{j, \alpha_{2}}^{a}\left(x_{2}\right)= & \sum_{p, \beta, t}{ }^{(1)} F_{c p}\left[\begin{array}{ll}
i & j \\
b & a
\end{array}\right]_{\alpha_{1} \alpha_{2}}^{\beta \quad} \sum_{P}\left\langle p, P\left|\phi_{i, t}\left(x_{12}\right)\right| j, 0\right\rangle{ }^{b} \Psi_{p, \beta ; P}^{a}\left(x_{2}\right) \\
& =\sum_{p, \beta, t}{ }^{(1)} F_{c p}\left[\begin{array}{ll}
i & j \\
b & a
\end{array}\right]_{\alpha_{1} \alpha_{2}}^{\beta} \frac{1}{\left|x_{12}\right|^{\triangle_{i}+\triangle_{j}-\triangle_{p}}}{ }^{b} \Psi_{p, \beta}^{a}\left(x_{2}\right)+\ldots,
\end{aligned}
$$

where $P$ is an index for the descendent states of the representation $\mathcal{V}_{p}$ with $p \in \mathcal{I}$. The indices $\alpha_{1}, \alpha_{2}, \beta$ account for the multiplicity of vertices of type $\left(\begin{array}{l}b \\ i c\end{array}\right),\left(\begin{array}{l}c \\ j a\end{array}\right),\left(\begin{array}{l}b \\ p a\end{array}\right)$, respectively, $a, b, c \in \mathcal{V}$, i.e., $\alpha_{1}=1,2 \ldots, n_{i c}{ }^{b}$, etc, while $t$ is that of a standard vertex $\left(\begin{array}{l}p \\ i j\end{array}\right), t=$ $1,2, \ldots N_{i j}{ }^{p}$. We will often restrict for simplicity to the $s l(2)$ case, so that the index $t$ can be omitted. From the 1-point function

$$
\begin{aligned}
& { }^{(1)} F_{c p}\left[\begin{array}{ll}
i & 1 \\
b & a
\end{array}\right]_{\alpha_{1} \alpha_{2}}^{\beta \quad}=\delta_{p i} \delta_{a c} \delta_{\alpha_{2} \mathbf{1}} \delta_{t \mathbf{1}} \delta_{\alpha_{1} \beta}, \\
& { }^{(1)} F_{c p}\left[\begin{array}{ll}
1 & j \\
b & a
\end{array}\right]_{\alpha_{1} \alpha_{2}}^{\beta}=\delta_{p j} \delta_{b c} \delta_{\alpha_{1} \mathbf{1}} \delta_{t \mathbf{1}} \delta_{\alpha_{2} \beta} .
\end{aligned}
$$

With the normalisation of the $\operatorname{CVO}\left(\begin{array}{c}p \\ i j\end{array}\right)_{t}$ implied by the second equality in (4.11) the numerical coefficients ${ }^{(1)} F_{c p}\left[\begin{array}{ll}i & j \\ b & a\end{array}\right]_{\alpha_{1} \alpha_{2}}^{\beta}$ with $i, j, p \in \mathcal{I}, a, b, c \in \mathcal{V}$, represent the OPE coefficients of the boundary fields and their determination is part of the problem. They are reminiscent of the matrix elements of the fusing (or crossing) matrices $F$ (whence the notation here for this "second" fusing matrix), which serve as OPE coefficients of the usual CVO [33]. The definition (4.11) extends to descendent fields in the l.h.s. as for the usual CVO. Symbolically (4.11) can be written as

$$
\left(\begin{array}{l}
b \\
i c
\end{array}\right)_{\alpha_{1}, x_{1}}\left(\begin{array}{l}
c \\
j a
\end{array}\right)_{\alpha_{2}, x_{2}}=\sum_{p, \beta, t}{ }^{(1)} F_{c p}\left[\begin{array}{ll}
i & j \\
b & a
\end{array}\right]_{\alpha_{1} \alpha_{2}}^{\beta}\left(\begin{array}{l}
p \\
i j
\end{array}\right)_{t, x_{12}}\left(\begin{array}{l}
b \\
p a
\end{array}\right)_{\beta, x_{2}}
$$

and can be depicted similarly as the standard Moore-Seiberg diagrams, see Figure Ð. Denoting by $\mathcal{U}_{p a}^{b}$ the space of boundary fields of type $\left(\begin{array}{l}b \\ p b\end{array}\right)_{\alpha_{1}}$ we have $\operatorname{dim} \mathcal{U}_{p a}^{b}=n_{p a}{ }^{b}$ while the space of standard CVO, $U_{i j}^{p}$, has dimension given by the Verlinde fusion multiplicity $N_{i j}{ }^{p}$, $\operatorname{dim} U_{i j}^{p}=N_{i j}{ }^{p}$. Thus we can interpret ${ }^{(1)} F$ as a linear operator

$$
\oplus_{c} \mathcal{U}_{i c}^{b} \otimes \mathcal{U}_{j a}^{c} \rightarrow \oplus_{p} U_{i j}^{p} \otimes \mathcal{U}_{p a}^{b}
$$

the dimension of the two sides being identical, according to (2.22). Given the 1- and 2-point correlators above the computation of the general boundary field $n$-point functions is reduced to the computation of the conformal blocks of the standard CVO $\left(\begin{array}{l}p \\ i j\end{array}\right)_{t, x}$. 


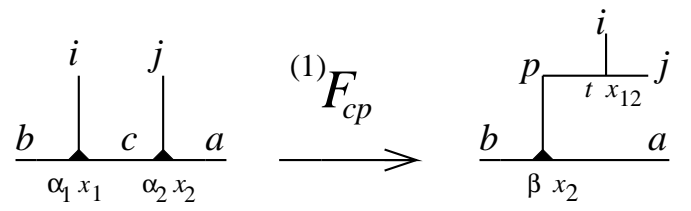

Figure 4: Graphical representation of (4.13). To stress the presence of two types of vertices, we distinguish them explicitly on this particular figure only

Comparing with the 2- and 3-point functions, see Figure 5(a) and (b), we have

$$
\begin{aligned}
& C_{i i^{*} ; \alpha_{1} \alpha_{2}}^{a b}={ }^{(1)} F_{b 1}\left[\begin{array}{cc}
i & i^{*} \\
a & a
\end{array}\right]_{\alpha_{1} \alpha_{2}}^{\mathbf{1}_{a} \mathbf{1}_{i}^{\prime}}\langle\mathbf{1}\rangle_{a} \\
& C_{i j k ; \alpha_{1} \alpha_{2} \alpha_{3} ; t}^{a b c}=\sum_{\beta}{ }^{(1)} F_{b k^{*}}\left[\begin{array}{cc}
i & j \\
a & c
\end{array}\right]_{\alpha_{1} \alpha_{2}}^{\beta \sigma_{23} \sigma_{13}(t)}{ }^{(1)} F_{c 1}\left[\begin{array}{rr}
k^{*} & k \\
a & a
\end{array}\right]_{\beta \alpha_{3}}^{\mathbf{1}_{a} \mathbf{1}_{k^{*}}^{\prime}}\langle\mathbf{1}\rangle_{a} \\
& =\sum_{\gamma}{ }^{(1)} F_{c i^{*}}\left[\begin{array}{ll}
j & k \\
b & a
\end{array}\right]_{\alpha_{2} \alpha_{3}}^{\gamma t}{ }^{(1)} F_{b 1}\left[\begin{array}{cc}
i & i^{*} \\
a & a
\end{array}\right]_{\alpha_{1} \gamma}^{\mathbf{1}_{a} \mathbf{1}_{i}^{\prime}}\langle\mathbf{1}\rangle_{a} \text {. }
\end{aligned}
$$

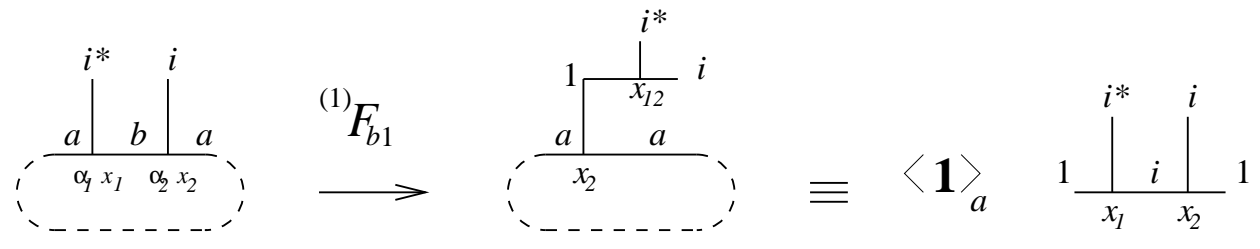

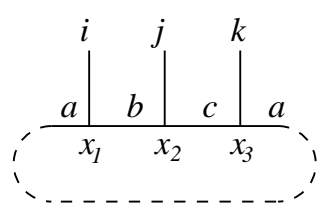
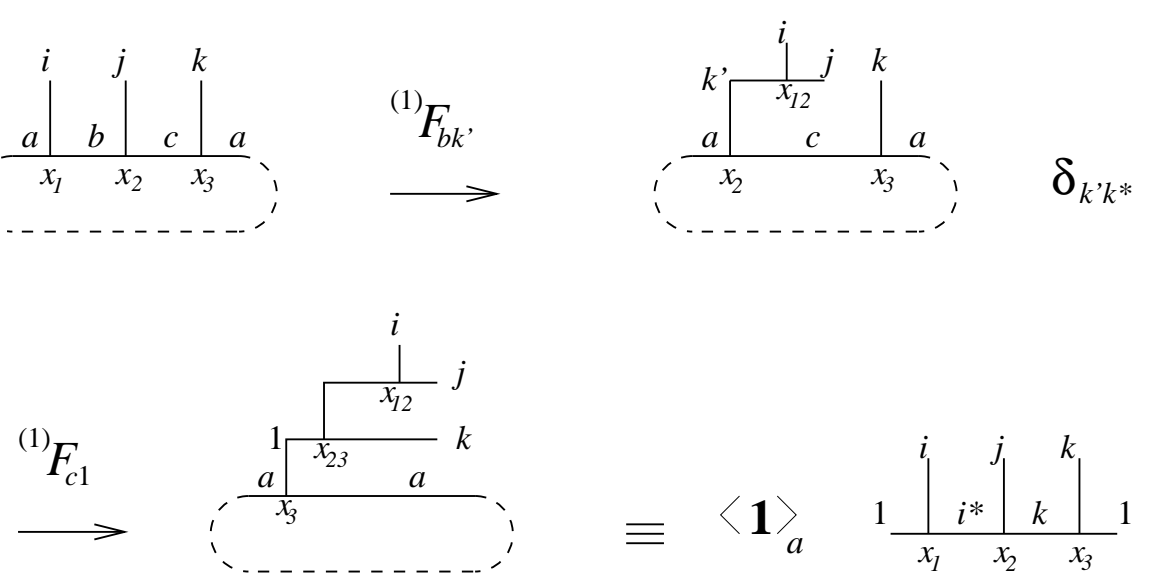

(b)

Figure 5: (a) and (b): boundary field 2- and 3-point functions

The 2- and 3-point normalisation coefficients are assumed to satisfy the symmetry 
conditions

$$
\begin{gathered}
C_{i^{*} i \alpha \beta}^{a b}=C_{i i^{*} ; \beta \alpha}^{b a}=\left(C_{i^{*} i ; \sigma_{13}\left(\beta^{*}\right) \sigma_{13}\left(\alpha^{*}\right)}^{a b}\right)^{*} \\
C_{i j k ; \alpha_{1} \alpha_{2} \alpha_{3} ; t}^{a b c}=C_{j k i ; \alpha_{2} \alpha_{3} \alpha_{1} ; \sigma_{13} \sigma_{23}(t)}^{b c a}=\left(C_{k^{*} j^{*} i^{*} ; \sigma_{13}\left(\alpha_{3}^{*}\right) \sigma_{13}\left(\alpha_{2}^{*}\right) \sigma_{13}\left(\alpha_{1}^{*}\right) ; \sigma_{13}\left(t^{*}\right)}^{c b a}\right)^{*}
\end{gathered}
$$

The first equalities in (4.17) are cyclic symmetry relations, see Figure 6, while the second equalities come from an antilinear ("CPT") transformation, which in particular sends the field ${ }^{b} \Psi_{j, \beta}^{c}(x)$ to its conjugate ${ }^{c} \Psi_{j, \sigma_{13}\left(\beta^{*}\right)}^{b}(-x)$ with multiplicity indices consistent with $n_{j a}{ }^{c}=$ $n_{j^{*} c}^{a}=n_{j^{*} a^{*}}{ }^{*}$.

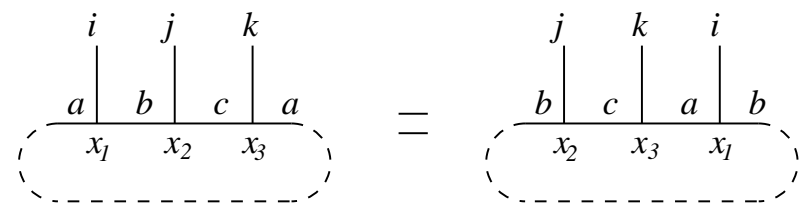

Figure 6: The cyclic symmetry of 3-point functions

The cyclic symmetry relations imply

$$
\begin{aligned}
{ }^{(1)} F_{c 1}\left[\begin{array}{cc}
j & j^{*} \\
a & a
\end{array}\right]_{\alpha_{1} \alpha_{2}}^{\mathbf{1}_{a} \mathbf{1}_{j}^{\prime}}\langle\mathbf{1}\rangle_{a}={ }^{(1)} F_{a 1}\left[\begin{array}{ll}
j^{*} & j \\
c & c
\end{array}\right]_{\alpha_{2} \alpha_{1}}^{\mathbf{1}_{c} \mathbf{1}_{j^{*}}^{\prime}}\langle\mathbf{1}\rangle_{c}, \\
\sum_{\beta}{ }^{(1)} F_{a k^{*}}\left[\begin{array}{ll}
j & s \\
b & c
\end{array}\right]_{\delta \alpha}^{\beta t}{ }^{(1)} F_{c 1}\left[\begin{array}{cc}
k^{*} & k \\
b & b
\end{array}\right]_{\beta \beta_{2}}^{\mathbf{1}_{b} \mathbf{1}_{k^{*}}^{\prime}}\langle\mathbf{1}\rangle_{b} \\
=\sum_{\gamma}{ }^{(1)} F_{b s^{*}}\left[\begin{array}{ll}
k & j \\
c & a
\end{array}\right]_{\beta_{2} \delta}^{\gamma \sigma_{23} \sigma_{13}(t)}{ }^{(1)} F_{c 1}\left[\begin{array}{cc}
s & s^{*} \\
a & a
\end{array}\right]_{\alpha \gamma}^{\mathbf{1}_{a} \mathbf{1}_{s}^{\prime}}\langle\rangle_{a},
\end{aligned}
$$

while the the second equalities in (4.17) lead to

$$
{ }^{(1)} F_{a k}\left[\begin{array}{ll}
j & s \\
b & c
\end{array}\right]_{\delta \alpha}^{\beta t}=\left({ }^{(1)} F_{a k^{*}}\left[\begin{array}{cc}
s^{*} & j^{*} \\
c & b
\end{array}\right]_{\sigma_{13}\left(\alpha^{*}\right) \sigma_{13}\left(\delta^{*}\right)}^{\sigma_{13}\left(\beta^{*}\right) \sigma_{23}\left(t^{*}\right)}\right)^{*} \text {. }
$$

Combining (4.18) and (4.19), one recovers (4.16).

\subsubsection{Bulk fields and bulk-boundary coefficients}

We turn to the second ingredient of the Cardy-Lewellen boundary CFT, the bulk fields. The half-plane bulk fields $\Phi_{I}(z, \bar{z}), z=x+i y \in H_{+}, \bar{z}=x-i y$ transform under a representation of $L^{(H)}$ 36 realised by differential operators

$$
L_{n}^{H}=L_{n}\left(\triangle_{i}, z\right)+L_{n}\left(\triangle_{\bar{i}}, \bar{z}\right)
$$


and characterised by a pair $I=(i, \bar{i})$ of weights. In cases when there is more than one field with the same labels $(i, \bar{i})$ a more involved notation like $I=(i, \bar{i} ; \alpha)$ is needed, but usually omitted for simplicity. For type I theories as well as for arbitrary scalar fields both $i, \bar{i} \in \mathcal{E}$, while in general $i, \bar{i} \in \mathcal{I}$.

The invariance with respect to the subalgebra spanned by $L_{ \pm 1,0}^{(H)}$ determines the 1-point function of $\Phi_{(i, \bar{i})}(z, \bar{z})$ as well as the 2 -point function $\left\langle{ }^{a} \Psi_{p, \alpha}^{a} \Phi_{(i, \bar{i})}\right\rangle$, e.g.,

$$
\begin{aligned}
& \left\langle\left. 0\right|^{a} \Psi_{p, \alpha}^{a}\left(x_{1}\right) \Phi_{(i, \bar{i})}(z, \bar{z}) \mid 0\right\rangle \\
& =\frac{C_{p,(i, \bar{i}), \alpha, t}^{a}}{(z-\bar{z})^{\triangle_{i}+\triangle_{\bar{i}}-\triangle_{p}}\left(x_{1}-z\right)^{\triangle_{i}+\triangle_{p}-\triangle_{\bar{i}}}\left(x_{1}-\bar{z}\right)^{\triangle_{\bar{i}}+\triangle_{p}-\triangle_{i}}}, \quad x_{1}>\operatorname{Re} z,
\end{aligned}
$$

while $\left\langle\Phi_{(i, \bar{i})}^{a} \Psi_{p, \alpha}^{a}\right\rangle$ is defined for Re $z>x_{1}$ by the analogous expression with $x_{1}-z, x_{1}-\bar{z}$ replaced by $z-x_{1}, \bar{z}-x_{1}$. Requiring the symmetry of this function under the exchange of the two fields, i.e., the independence of the ordering, leads to the constraint $\triangle_{i}-\triangle_{\bar{i}} \in \mathbb{Z}$.

The r.h.s. of (4.22) is the 3-point block of the standard CVO $\left\langle 0\left|\phi_{p, \mathbf{1}_{p}^{\prime}}\left(x_{1}\right) \phi_{i, t}(z) \phi_{\bar{i}, \mathbf{1}_{\bar{i}}}(\bar{z})\right| 0\right\rangle$, with $t$ a coupling index of type $\left(\begin{array}{c}p^{*} \\ i \bar{i}\end{array}\right), t=1,2, \ldots, N_{i \bar{i}} p^{*}$. Consistently with this the (primary) bulk field can be represented for small $z-\bar{z}$ via the decomposition

$$
\begin{aligned}
\Phi_{(i, \bar{i})}(z, \bar{z}) & =\sum_{a, \alpha, p \in \mathcal{I}, t}{ }^{a, \alpha} B_{(i, \bar{i})}^{p, t} \sum_{P}\left\langle p, P\left|\phi_{i, t}(z-\bar{z})\right| \bar{i}, 0\right\rangle{ }^{a} \Psi_{p, \alpha ; P}^{a}(\bar{z}) \\
& =\sum_{a, \alpha, p \in \mathcal{I}, t}{ }^{a, \alpha} B_{(i, \bar{i})}^{p, t} \frac{1}{(z-\bar{z})^{\triangle_{i}+\triangle_{\bar{i}}-\triangle_{p}}}{ }^{a} \Psi_{p, \alpha}^{a}(\bar{z})+\ldots \\
& =\sum_{a, \alpha, p \in \mathcal{I}, t}{ }^{a, \alpha} B_{(i, \bar{i})}^{p, t} \frac{1}{(2 i y)^{\triangle_{i}+\triangle_{\bar{i}}-\triangle_{p}}}{ }^{a} \Psi_{p, \alpha}^{a}(x)+\ldots
\end{aligned}
$$

which extends to descendents. Here ${ }^{a} \Psi_{p, \alpha}^{a}(z)$ are "unphysical" generalised CVO obtained extending to the (full) plane the boundary fields of the previous section. Their OPE is determined by the same fusing matrix ${ }^{(1)} F$, i.e., as in (4.11), the latter extended to complex $\operatorname{arguments} z_{i}$, Re $z_{12}>0$, with $\left|x_{12}\right|$ replaced by $z_{12}$; we shall need only this fusing property.

The constants ${ }^{a, \alpha} B_{(i, \bar{i})}^{p, t}$ ("bulk-boundary reflection coefficients") in this decomposition depend on two couplings of different types, $\left(\begin{array}{l}p \\ i \bar{i}\end{array}\right)_{t}$ and $\left(\begin{array}{l}a \\ p a\end{array}\right)_{\alpha}$. Note that the coefficients used here differ by a phase from the traditionally normalised coefficients [11], [12], which will be denoted ${ }^{a, \alpha} B_{(i, \bar{i})}^{p, t}(C L)$, i.e.,

$$
{ }^{a, \alpha} B_{(i, \bar{i})}^{p, t}=e^{i \frac{\pi}{2}\left(\triangle_{i}+\triangle_{\bar{i}}-\triangle_{p}\right) a, \alpha} B_{(i, \bar{i})}^{p, t}(C L) .
$$

The decomposition (4.23), symbolically written as

$$
\Phi_{(i, \bar{i})}(z, \bar{z})=\sum_{a, \alpha, p, t}{ }^{a, \alpha} B_{(i, \bar{i})}^{p, t}\left(\begin{array}{l}
p \\
i \bar{i}
\end{array}\right)_{t, z-\bar{z}}\left(\begin{array}{l}
a \\
p a
\end{array}\right)_{\alpha, \bar{z}}
$$

see Figure 7, reduces the computation of the $n$-point functions of $\Phi_{(i, \bar{i})}$ to the computation of the blocks of the generalised CVO ${ }^{a} \Psi_{p, \alpha}^{b}(z)$, which combined with their OPE (the extension 
from $\left|x_{12}\right|$ to $z_{12}$ of (4.11) ) allows to recover all correlators in terms of standard conformal blocks. The invariant 1-point function projected onto the boundary state $a$ reads

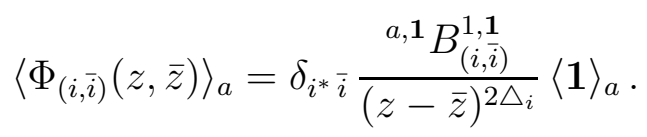

Omitting the trivial indices and simplifying the label $\left(i, i^{*}\right)$ to $i$, one has in particular ${ }^{a} B_{1}^{1}=1$ for any $a$.

The OPE of the half-plane bulk fields $\Phi_{(k, \bar{k})}(z, \bar{z})$ is defined according to

$$
\begin{aligned}
& \Phi_{(k, \bar{k})}\left(z_{1}, \bar{z}_{1}\right) \Phi_{(l, \bar{l})}\left(z_{2}, \bar{z}_{2}\right) \\
& =\sum_{j, \bar{j}, t, \bar{t}} D_{(k, \bar{k})(l, \bar{l})}^{(j, \bar{t}): \bar{t}} \sum_{J, \bar{J}}\left\langle j, J\left|\phi_{k, t}\left(z_{12}\right)\right| l, 0\right\rangle\left\langle\bar{j}, \bar{J}\left|\phi_{\bar{k}, \bar{t}}\left(\bar{z}_{12}\right)\right| \bar{l}, 0\right\rangle \Phi_{(j, \bar{j}) ;(J, \bar{J})}\left(z_{2}, \bar{z}_{2}\right) \\
& =\sum_{j, \bar{j}, t, \bar{t}} \frac{D_{(k, \bar{k})(l, \bar{l})}^{(j, \bar{j}) ; t, \bar{t}}}{{z_{12}^{k}}_{k l}^{j} \bar{z}_{12}^{\triangle_{\bar{k}}} \bar{l}} \Phi_{(j, \bar{j})}\left(z_{2}, \bar{z}_{2}\right)+\ldots
\end{aligned}
$$

The coefficients $D_{(k, \bar{k})(l, \bar{l})}^{(j, \bar{j}) ; t \bar{t}}$ are related to the full-plane bulk OPE coefficients, see below.

\subsection{Boundary CFT duality relations}

\subsubsection{Cardy-Lewellen equations rederived}

We collect in this section the set of equations resulting from the sewing constraints on the various OPE expansions [12]; some of these equations can be interpreted as expressing locality (symmetry) of the boundary CFT correlators. For simplicity of notation we shall sometimes omit the explicit indication of the coupling indices of type $\left(\begin{array}{l}i \\ j k\end{array}\right)$ and the corresponding summations, i.e., the equations will be written essentially for the simplest $s l(2)$ case. However we shall keep the charge conjugation in the indices of $\mathcal{I}$ so that the general formulae can be easily recovered. In the $\operatorname{sl}(2) \mathrm{WZW}$ case the braiding phases are given by the shifted scaling dimensions $\triangle_{j}^{S u g}-j$, instead of $\triangle_{j}^{S u g}$ (since the pair of coordinate and isospin variables is moved as a whole). Then formulae work equally well with the same fusing and braiding matrices, without additional signs, as for the corresponding subfamily $(1,2 j+1)$ of fields in Virasoro minimal models.

Applying (4.11) in different ways to the 4-point function of boundary fields $\Psi$, that is demanding associativity, leads to a relation connecting the two types of fusing matrices ${ }^{(1)} F$

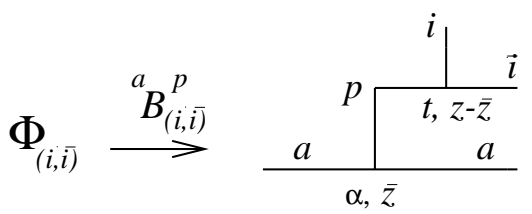

Figure 7: Graphical representation of the decomposition (4.25) of bulk fields 
and $F$, the fusing matrix for the ordinary CVO, which reads symbolically

$$
F^{(1)} F^{(1)} F={ }^{(1)} F^{(1)} F
$$

or, more explicitly,

$$
\begin{gathered}
\sum_{m, \beta_{2}, t_{3}, t_{2}} F_{m p}\left[\begin{array}{ll}
i & j \\
l & k
\end{array}\right]_{t_{2} t_{3}}^{u_{2} u_{3}}{ }^{(1)} F_{b l}\left[\begin{array}{cc}
i & m \\
a & d
\end{array}\right]_{\alpha_{1} \beta_{2}}^{\gamma_{1} t_{2}}{ }^{(1)} F_{c m}\left[\begin{array}{ll}
j & k \\
b & d
\end{array}\right]_{\alpha_{2} \alpha_{3}}^{\beta_{2} t_{3}} \\
=\sum_{\beta_{1}}{ }^{(1)} F_{c l}\left[\begin{array}{ll}
p & k \\
a & d
\end{array}\right]_{\beta_{1} \alpha_{3}}^{\gamma_{1} u_{2}}{ }^{(1)} F_{b p}\left[\begin{array}{ll}
i & j \\
a & c
\end{array}\right]_{\alpha_{1} \alpha_{2}}^{\beta_{1} u_{3}}
\end{gathered}
$$

The identity (4.29), when restricted to the $\operatorname{sl}(2)$ case, is a slightly simplified version of the equation ( $\mathrm{L}$ 3.29) in [12] and can be also obtained from the latter using the relation (4.16) and dropping a (non-zero) factor of type ${ }^{(1)} F_{a 1}$. The direct derivation of this pentagon-like identity depicted in Figure 8 is analogous to the derivation of the standard pentagon equation for the fusing matrices $F$ since the boundary field $n$-point blocks are analogs of the ordinary $(n+2)$-point conformal blocks with an additional constraint due to the delta function in the 2-point boundary block. T The relation (4.16) is reproduced from the pentagon identity (4.29) for particular values of the indices.

Imposing the symmetry of the 3-point function $\left\langle{ }^{b} \Psi_{j, \delta}^{a}\left(x_{1}\right) \Phi_{(i, \bar{i})}(z, \bar{z})^{a} \Psi_{k, \gamma}^{b}\left(x_{2}\right)\right\rangle=$ $\left\langle{ }^{b} \Psi_{j, \delta}^{a}\left(x_{1}\right){ }^{a} \Psi_{k, \gamma}^{b}\left(x_{2}\right) \Phi_{(i, \bar{i})}(z, \bar{z})\right\rangle$ one derives following Figure 9,

$$
\begin{aligned}
& \sum_{\beta, \beta^{\prime}}{ }^{b, \beta} B_{(i, \bar{i})}^{p}\langle\mathbf{1}\rangle_{b}{ }^{(1)} F_{b 1}\left[\begin{array}{rr}
p^{*} & p \\
b & b
\end{array}\right]_{\beta^{\prime} \beta}^{\mathbf{1}}{ }^{(1)} F_{a p^{*}}\left[\begin{array}{ll}
j & k \\
b & b
\end{array}\right]_{\delta \gamma}^{\beta^{\prime}}=\sum_{s, \alpha, \alpha^{\prime}}{ }^{a, \alpha} B_{(i, \bar{i})}^{s}\langle\mathbf{1}\rangle_{a}{ }^{(1)} F_{a 1}\left[\begin{array}{cc}
s^{*} & s \\
a & a
\end{array}\right]_{\alpha^{\prime} \alpha}^{\mathbf{1}} \\
& { }^{(1)} F_{b s^{*}}\left[\begin{array}{ll}
k & j \\
a & a
\end{array}\right]_{\gamma \delta}^{\alpha^{\prime}} \sum_{m} e^{i \pi\left(2 \triangle_{i}-2 \triangle_{m}+\triangle_{k}+\triangle_{j}-\triangle_{p}\right)} F_{s m}\left[\begin{array}{cc}
j & i \\
k^{*} & \bar{i}
\end{array}\right] F_{m p^{*}}\left[\begin{array}{cc}
k & j \\
\bar{i}^{*} & i
\end{array}\right] .
\end{aligned}
$$

On Figure 9 the braiding matrices $B( \pm)$ appear, see Appendix E. In the r.h.s. of (4.30) we have also used the cyclic symmetry relations $(4.18),(4.19)$. Using furthermore these relations a factor of type ${ }^{(1)} F_{a 1}$ (and the related summation) can be dropped in both sides of (4.30) which leads to a slighly simplified version as compared with the original equation (L 3.32) in 12$]$.

From the 2-point function $\left\langle\Phi_{(k, \bar{k})}\left(z_{1}, \bar{z}_{1}\right) \Phi_{(l, \bar{l})}\left(z_{2}, \bar{z}_{2}\right)\right\rangle_{a}$ using either the OPE formula (4.27), or (4.23) (follow Figure 10 with $i=1$ ), we obtain

$$
\begin{aligned}
& \sum_{\alpha, \beta}{ }^{a, \alpha} B_{(k, \bar{k})}^{r, s_{1}}{ }^{a, \beta} B_{(l, \bar{l})}^{r^{*}, s_{2}}{ }^{(1)} F_{a 1}\left[\begin{array}{cc}
r & r^{*} \\
a & a
\end{array}\right]_{\alpha \beta}^{\mathbf{1}_{a} \mathbf{1}_{r}^{\prime}} \\
& =\sum_{j}{ }^{a} B_{j}^{1} e^{i \pi\left(\triangle_{k}+\triangle_{\bar{l}}-\triangle_{r}-\triangle_{j}\right)} \sum_{t, \bar{t}} D_{(k, \bar{k})(l, \bar{l})}^{(j, j)} F_{j r}^{*}\left[\begin{array}{cc}
\bar{k} & k \\
l^{*} & l
\end{array}\right]_{\sigma_{13}(\bar{t}) \quad t}^{\sigma_{23} \sigma_{13}\left(s_{2}\right) \sigma_{23}\left(s_{1}\right)},
\end{aligned}
$$

\footnotetext{
4'Mixed' pentagon identities analogous to 4.28) appear in the framework of 'weak Hopf algebras' as part of a 'Big Pentagon identity' 65. The counterparts of ${ }^{(1)} F$ are interpreted as kind of " $3 \mathrm{j}$ - symbols" along with the standard interpretation of the fusing matrices $F$ as " $6 \mathrm{j}$ - symbols".
} 


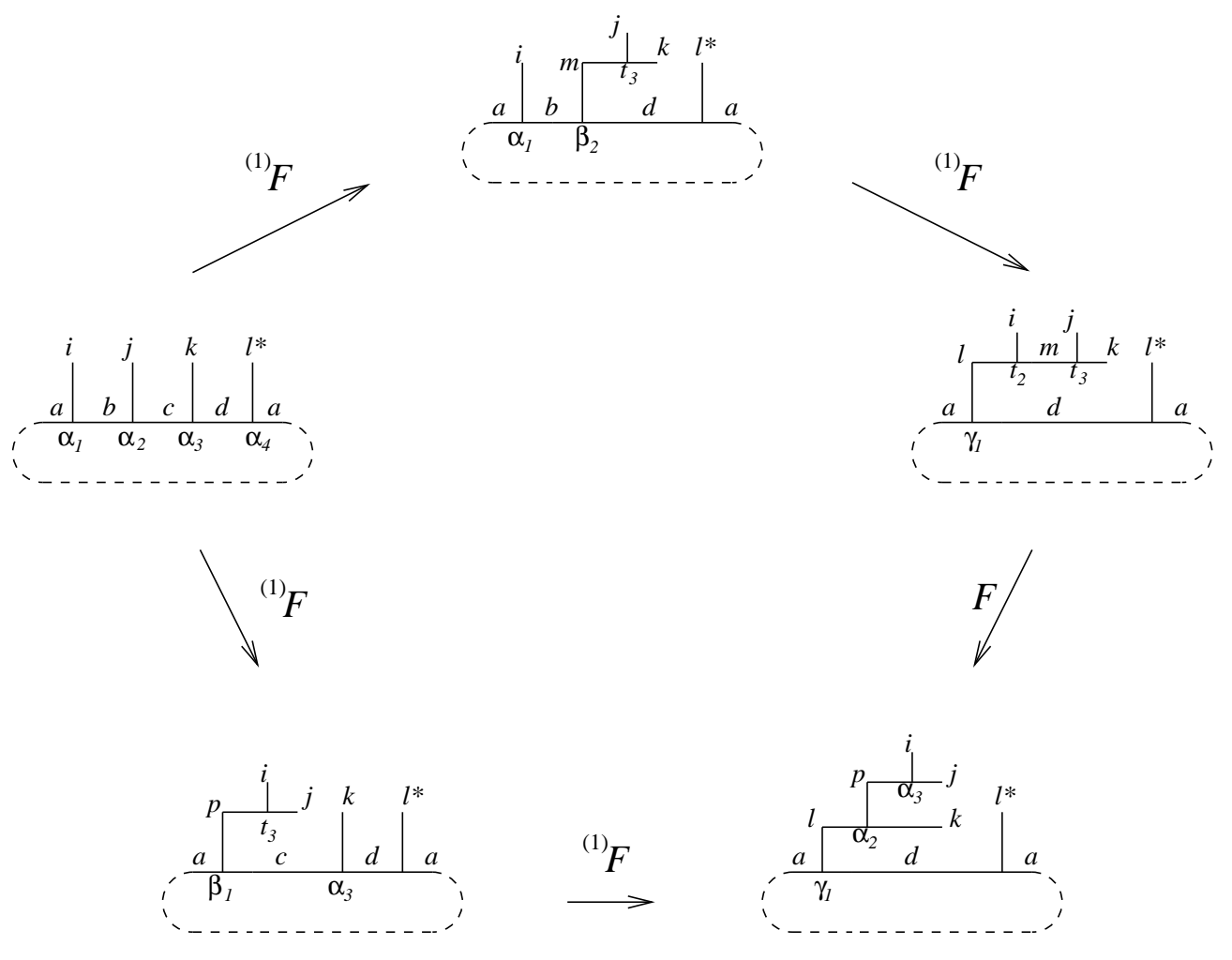

Figure 8: The "mixed" pentagon identity

or, equivalently,

$$
\begin{aligned}
& D_{(k, \bar{k})(l, \bar{l})}^{\left(j, j^{*}\right) ;, \bar{t}}{ }^{a} B_{j}^{1} e^{i \pi\left(\triangle_{k}+\triangle_{\bar{l}}-\triangle_{j}\right)} \\
& =\sum_{\alpha, \beta, r, s_{1}, s_{2}} e^{i \pi \triangle_{r} a, \alpha} B_{(k, \bar{k})}^{r, s_{1}}{ }^{a, \beta} B_{(l, \bar{l})}^{r^{*}, s_{2}(1)} F_{a 1}\left[\begin{array}{cc}
r & r^{*} \\
a & a
\end{array}\right]_{\alpha \beta}^{\mathbf{1}_{a} \mathbf{1}_{r}^{\prime}} F_{r^{*} j}\left[\begin{array}{cc}
k & l \\
\bar{k}^{*} & \bar{l}
\end{array}\right]_{\sigma_{13}\left(s_{1}\right)}^{\sigma_{12}(\bar{t}) t}
\end{aligned}
$$

Lastly from the 3-point function $\left\langle\Phi_{(k, \bar{k})}\left(z_{1}\right) \Phi_{(l, \bar{l})}\left(z_{2}\right)^{a} \Psi_{i, \gamma}^{a}(x)\right\rangle$, we obtain, see Figure 10

$$
\begin{aligned}
& \sum_{\alpha, \beta}^{a, \alpha} B_{(k, \bar{k})}^{r}{ }^{a, \beta} B_{(l, \bar{l})}^{t}{ }^{(1)} F_{a i^{*}}\left[\begin{array}{cc}
r & t \\
a & a
\end{array}\right]_{\alpha \beta}^{\gamma} \\
& =\sum_{j, \bar{j}} D_{(k, \bar{k})(l, \bar{l})}^{(j, \bar{j})}{ }^{a, \gamma} B_{(j, \bar{j})}^{i} e^{i \pi\left(\triangle_{k}-\triangle_{r}-\triangle_{j}\right)} \sum_{s} e^{i \pi \triangle_{s}} F_{\bar{j} s^{*}}\left[\begin{array}{cc}
j & \bar{k} \\
i^{*} & \bar{l}
\end{array}\right] F_{j^{*} r^{*}}\left[\begin{array}{cc}
l & s \\
k^{*} & \bar{k}
\end{array}\right] F_{s t}\left[\begin{array}{cc}
l & \bar{l} \\
r^{*} & i
\end{array}\right]
\end{aligned}
$$

For $i=1$ (4.33) reduces to (4.31). The sum over $s$ in the r.h.s. of (4.33) represents up to phases one of the sides in (an auxiliary) hexagon identity, resulting in permuting $\{l, i, \bar{k}\}$ to $\{\bar{k}, i, l\}$ i.e., can be written as $B_{23}(-) B_{12}(-) B_{23}(-)$ and thus can be replaced by $B_{12}(-) B_{23}(-) B_{12}(-)$. This gives an alternative representation of the r.h.s. of (4.33) obtained from the above by replacing everywhere $(k, \bar{k}, j, r) \rightarrow\left(\bar{l}, l, \bar{j}, t^{*}\right)$. This is the original form of the equation ( $\mathrm{L} 3.35)$ in [12], when (4.24) is inserted, with furthermore inverse operator ordering convention and opposite overall sign of the phase. 

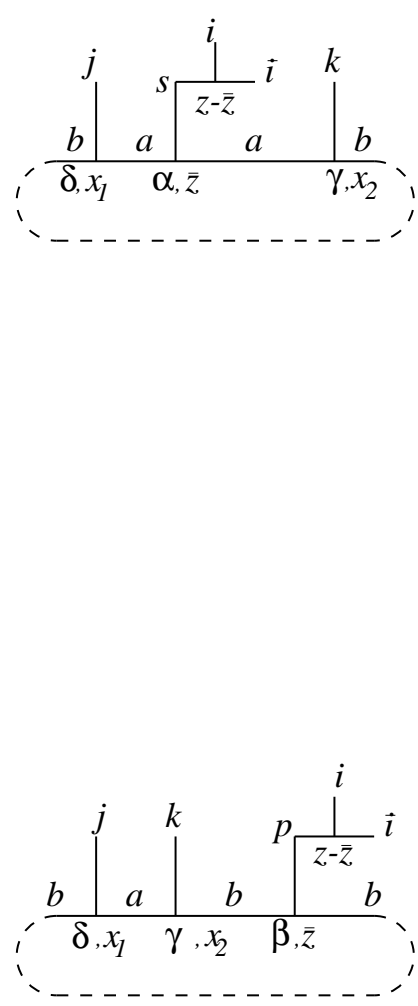
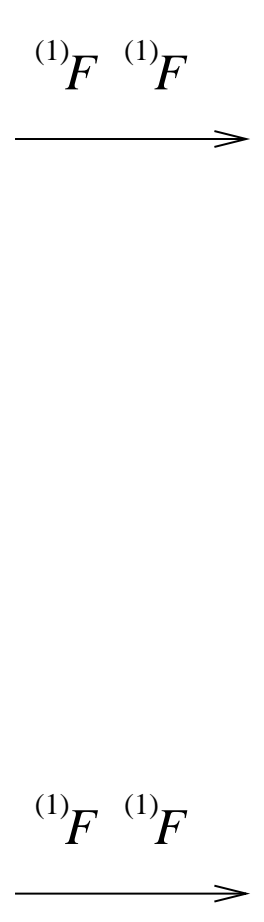
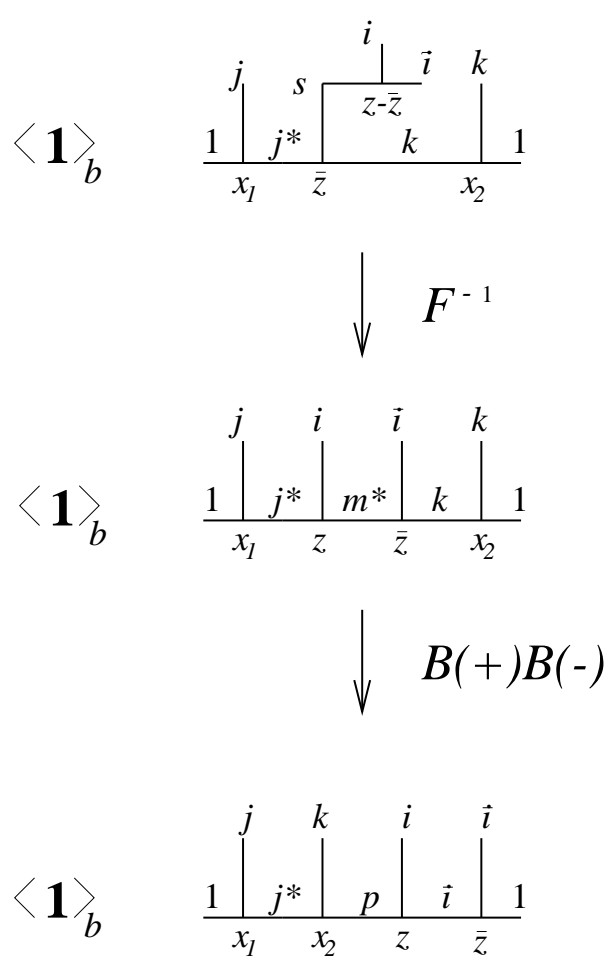

Figure 9: Derivation of $(4.30)$

The locality of this 3-point function (the symmetry under the exchange of the fields $\Phi$ ) implies also

$$
D_{(k, \bar{k})(l, \bar{l})}^{(j, \bar{t}): t \bar{t}}=(-1)^{s_{k}+s_{l}-s_{j}} D_{(l, \bar{l})(k, \bar{k})}^{(j, \bar{j}) ; \sigma_{23}(t), \sigma_{23}(\bar{t})}, \quad s_{k}:=\triangle_{k}-\triangle_{\bar{k}}
$$

while from the associativity of the OPE (4.27) one obtains in particular the relation

$$
D_{(k, \bar{k})(l, \bar{l})}^{(j, \bar{j}) ; t \bar{t}} D_{(j, \bar{j})\left(j^{*}, \bar{j}^{*}\right)}^{(1,1)}=D_{(l, \bar{l})\left(j^{*}, \bar{j}^{*}\right)}^{\left(k^{*}, \bar{k}^{*}\right) \sigma_{13} \sigma_{23}(t), \sigma_{13} \sigma_{23}(\bar{t})} D_{\left(k^{*}, \bar{k}^{*}\right)(k, \bar{k})}^{(1,1)}
$$

All the above equations hold true as well with a $\operatorname{sign} \epsilon= \pm 1$, inserted in the exponents of all the phases in these equations including (4.24), and replacing the bulk-boundary coefficients $B$ with $B_{\epsilon}$. Thus when rewritten in terms of the Cardy-Lewellen normalised coefficients $B(C L)$ all equalities are true for both choices of sign.

Remark: The Lewellen equations in the diagonal $s l(2)$ case were recently confirmed in [70]. The seemingly different version of equation (4.30) in [14 is in fact equivalent to the original Lewellen equation, after taking into account one of the duality relations (a hexagon identity, see (E.6) in Appendix E) for the braiding matrices. On the other hand the derivation of the versions of (4.31), (4.33) in [14] appears to be affected by a missing phase in the intermediate (and needless) formula (17) in [14. This phase is compensated in the final formulae following from (4.31) (like (4.44) below) by another phase due to the presumably neglected difference in the normalisation of the bulk-boundary coefficients (like in (4.24)) as compared with that in [11, 12]. 

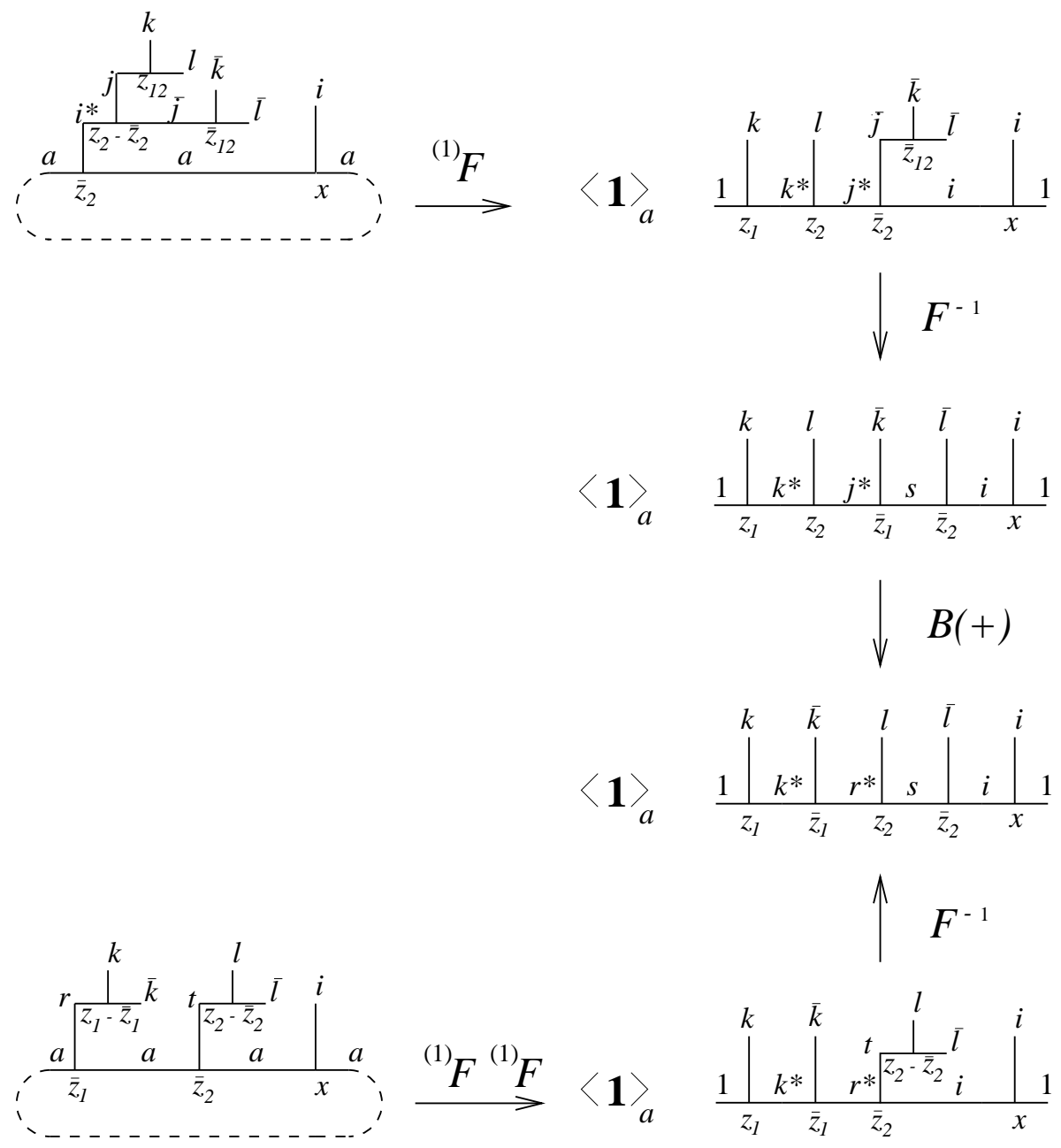

Figure 10: Derivation of $(4.33)$

\subsubsection{More pentagon relations}

Before we turn to a discussion on the implications of the Cardy-Lewellen equations we shall introduce one more ingredient to the scheme. It is natural to assume that there exists a "third fusing matrix", a matrix inverting ${ }^{(1)} F,{ }^{(3)} F^{(1)} F=I={ }^{(1)} F{ }^{(3)} F$, or more explicitly,

$$
\begin{gathered}
\sum_{b, \beta_{2}, \beta_{3}}{ }^{(3)} F_{p b^{*}}\left[\begin{array}{ll}
c^{*} & k \\
a^{*} & j
\end{array}\right]_{\sigma_{23}\left(\alpha_{2}\right) t^{\prime}}^{\sigma_{12}\left(\beta_{3}\right) \sigma_{12}\left(\beta_{2}\right)}{ }^{(1)} F_{b s}\left[\begin{array}{ll}
k & j \\
c & a
\end{array}\right]_{\beta_{2} \beta_{3}}^{\sigma_{13}\left(\gamma_{2}\right) t}=\delta_{p s} \delta_{\alpha_{2} \gamma_{2}} \delta_{t^{\prime} t}, \\
{ }^{(3)} F_{p b}\left[\begin{array}{ll}
a & 1 \\
c & k
\end{array}\right]_{\alpha t}^{\beta \gamma}=\delta_{a b} \delta_{k p} \delta_{\alpha \beta} \delta_{t \mathbf{1}} \delta_{\gamma \mathbf{1}}, \\
{ }^{(3)} F_{p b}\left[\begin{array}{ll}
a & j \\
c & 1
\end{array}\right]_{\alpha t}^{\beta \gamma}=\delta_{c b} \delta_{j p} \delta_{\alpha \gamma} \delta_{t \mathbf{1}} \delta_{\beta \mathbf{1}} .
\end{gathered}
$$


Along with the standard CVOs $\left(\begin{array}{l}p \\ j k\end{array}\right)$, this matrix involves new "couplings" of type $\left(\begin{array}{l}c \\ a p\end{array}\right)_{\beta^{\prime}}$ which can be thought of as obtained by a permutation $\sigma_{23}$ from the boundary fields $\left(\begin{array}{l}c \\ p a\end{array}\right)_{\beta}$, whence the notation $\beta^{\prime}=\sigma_{23}(\beta)$. The matrix ${ }^{(3)} F$ satisfies a "mixed" pentagon identity analogous to 4.28

$$
{ }^{(3)} F^{(3)} F F={ }^{(3)} F^{(3)} F \text {. }
$$

Furthermore multiplying both sides of (4.29) with ${ }^{(3)} F_{m^{\prime} c^{*}}\left[\begin{array}{ll}b^{*} & j \\ d^{*} & k\end{array}\right]_{\delta t}^{\sigma_{12}\left(\alpha_{3}\right) \sigma_{12}\left(\alpha_{2}\right)}$ and summing over $c, \alpha_{2}, \alpha_{3}$, using (4.36) in the r.h.s., we obtain another equation of similar form

$$
{ }^{(3)} F^{(1)} F^{(1)} F={ }^{(1)} F F \text {, }
$$

which implies various useful relations obtained for particular values of the indices. One of them reproduces the inverse property of ${ }^{(3)} F$, another one reads

$$
\sum_{\beta}{ }^{(3)} F_{1 b^{*}}\left[\begin{array}{cc}
a^{*} & j^{*} \\
a^{*} & j
\end{array}\right]_{11}^{\sigma_{23}(\alpha) \sigma_{23}(\beta)}{ }^{(1)} F_{a 1}\left[\begin{array}{cc}
j & j^{*} \\
b & b
\end{array}\right]_{\sigma_{13}(\gamma) \sigma_{13}(\beta)}^{\mathbf{1 1}}=\frac{1}{d_{j}} \delta_{\alpha, \gamma}
$$

where $d_{j}=S_{j 1} / S_{11}=F_{11}\left[\begin{array}{cc}j & j^{*} \\ j & j\end{array}\right]^{-1}$ is the quantum dimension. It furthermore implies

$$
d_{j} \sum_{\alpha, \beta}{ }^{(3)} F_{1 b^{*}}\left[\begin{array}{ll}
a^{*} & j^{*} \\
a^{*} & j
\end{array}\right]_{11}^{\sigma_{23}(\alpha) \sigma_{23}(\beta)}{ }^{(1)} F_{a 1}\left[\begin{array}{ll}
j & j^{*} \\
b & b
\end{array}\right]_{\sigma_{13}(\alpha) \sigma_{13}(\beta)}^{11}=n_{j a}^{b}
$$

Using the inverse matrix ${ }^{(3)} F$, as introduced here, the half-plane bulk field can be also written as a product of generalised boundary fields (a "bilocal" operator)

$$
\Phi_{(i, \bar{i})}^{H}(z, \bar{z})=\sum_{a, b, \alpha, \beta}\left(\sum_{p, \gamma, t}^{a, \gamma} B_{(i, \bar{i})}^{p, t}{ }^{(3)} F_{p b^{*}}\left[\begin{array}{ll}
a^{*} & i \\
a^{*} & \bar{i}
\end{array}\right]_{\sigma_{12} \sigma_{23}(\gamma) t}^{\sigma_{12} \sigma_{23}(\beta) \sigma_{12} \sigma_{23}(\alpha)}\right)^{a} \Psi_{i, \alpha}^{b}(z)^{b} \Psi_{\bar{i}, \beta}^{a}(\bar{z}),
$$

which reproduces the small $z-\bar{z}$ expansion in 4.23$)$; compare (4.40) with the chiral decomposition of the (full) plane physical fields

$$
\Phi_{(i, \bar{i})}^{P}(\zeta, \bar{\zeta})=\sum_{k, \bar{k}, l, \bar{l}, t, t^{\prime}}{ }^{(p l)} D_{(j, \bar{j})(k, \bar{k})}^{(l, \bar{l}) ;\left(t, t^{\prime}\right)}{ }^{l} \phi_{i, t}^{k}(\zeta) \otimes{ }^{\bar{l}} \phi_{\bar{i}, t^{\prime}}^{\bar{k}}(\bar{\zeta})
$$

Finally we shall exploit the inverse ${ }^{(3)} F$ of the matrix ${ }^{(1)} F$ to rewrite 4.30 ) in another equivalent form to be used in the next section. Namely we apply the inverse to ${ }^{(1)} F_{b s^{*}}$ in the r.h.s. and obtain

$$
\sum_{\alpha}{ }^{a, \alpha} B_{(i, \bar{i})}^{s}\langle\mathbf{1}\rangle_{a}{ }^{(1)} F_{a 1}\left[\begin{array}{cc}
s^{*} & s \\
a & a
\end{array}\right]_{\sigma_{13}\left(\alpha^{\prime}\right) \alpha}^{1} \sum_{m} e^{i \pi\left(2 \triangle_{i}-2 \triangle_{m}+\triangle_{k}+\triangle_{j}-\triangle_{p}\right)} F_{s m}\left[\begin{array}{cc}
j & i \\
k^{*} & \bar{i}
\end{array}\right] F_{m p^{*}}\left[\begin{array}{cc}
k & j \\
\bar{i}^{*} & i
\end{array}\right]
$$




$$
\begin{aligned}
& =\sum_{b, \beta, \beta^{\prime}}{ }_{b, \beta} B_{(i, \bar{i})}^{p}\langle\mathbf{1}\rangle_{b}{ }^{(1)} F_{b 1}\left[\begin{array}{rr}
p^{*} & p \\
b & b
\end{array}\right]_{\beta^{\prime} \beta}^{\mathbf{1}} \sum_{\gamma, \delta}{ }^{(3)} F_{s^{*} b^{*}}\left[\begin{array}{ll}
a^{*} & k \\
a^{*} & j
\end{array}\right]_{\sigma_{23}\left(\alpha^{\prime}\right)}^{\sigma_{23}(\delta) \sigma_{23}(\gamma)}{ }^{(1)} F_{a p^{*}}\left[\begin{array}{ll}
j & k \\
b & b
\end{array}\right]_{\sigma_{13}(\delta) \sigma_{13}(\gamma)}^{\beta^{\prime}} \\
& =\sum_{b, \beta, \gamma^{\prime}, \delta}{ }^{b, \beta} B_{(i, \bar{i})}^{p}\langle\mathbf{1}\rangle_{b}{ }^{(1)} F_{b k^{*}}\left[\begin{array}{rr}
p & j \\
b & a
\end{array}\right]_{\beta \sigma_{13}(\delta)}^{\gamma^{\prime}} \sum_{\gamma}^{(3)} F_{s^{*} b^{*}}\left[\begin{array}{ll}
a^{*} & k \\
a^{*} & j
\end{array}\right]_{\sigma_{23}\left(\alpha^{\prime}\right)}^{\sigma_{23}(\delta) \sigma_{23}(\gamma)}{ }^{(1)} F_{a 1}\left[\begin{array}{rr}
k^{*} & k \\
b & b
\end{array}\right]_{\gamma^{\prime} \sigma_{13}(\gamma)}^{\mathbf{1}} .
\end{aligned}
$$

In the third line we have used the symmetry relation (4.16).

\subsection{Consequences of the bulk-boundary equations}

\subsubsection{The Pasquier algebra and its dual}

In this section we analyse some important consequences of the set of equations derived. We start with equation (4.41), an inverted version of the first Lewellen bulk-boundary equation (4.30), in which we take $s=1=p$. This implies $k=j^{*}, \bar{i}=i^{*}, \alpha=\mathbf{1}=\alpha^{\prime}=\beta=\beta^{\prime}$. The sum over $m$ in the l.h.s. is proportional to the modular matrix $S_{j i}$, see $(\mathbb{E} .9)$, while the sums over the coupling indices $\delta, \gamma$ are worked out using (4.39), the final result being

$$
\frac{S_{j i}}{S_{1 i}}{ }^{a} B_{i}^{1}\langle\mathbf{1}\rangle_{a}=\sum_{b} n_{j a}{ }^{b}{ }^{b} B_{i}^{1}\langle\mathbf{1}\rangle_{b} .
$$

For simplicity we have done this computation in the $s l(2)$ case but it extends straightforwardly to arbitrary rank leading to the same formula.

Comparing (4.42) with (3.18), we see that it can be identified with the realisation (3.18) of the relation (3.4) in terms of the eigenvalues $\hat{\gamma}_{a}(i)=\frac{\psi_{a}^{i}}{\psi_{1}^{2}}$ of the graph algebra matrices $\hat{N}_{a}$. Namely we can identify the ratio ${ }^{b} B_{i}^{1}\langle\mathbf{1}\rangle_{b} /{ }^{a} B_{i}^{1}\langle\mathbf{1}\rangle_{a}$ with the ratio $\hat{\gamma}_{b}(i) / \hat{\gamma}_{a}(i)$. Recalling the expression for $\langle\mathbf{1}\rangle_{a}$ in (4.6) we find a relation between the boundary state coefficients $\psi_{a}^{i}$ and the bulk-boundary coefficients ${ }^{a} B_{i}^{1}$

$$
{ }^{a} B_{i}^{1}=e^{i \pi \triangle_{i} a} B_{i}^{1}(C L)=\frac{\psi_{a}^{i}}{\psi_{a}^{1}} e^{i \pi \triangle_{i}} \sqrt{\frac{C_{i i^{*}}}{d_{i}}},
$$

where for the time being $C_{i i^{*}}$ is an arbitrary constant, $C_{11}=1$. Conversely, if we assume the identification (4.43) (as, e.g., derived in the $s l(2)$ case by other means in [11, with $C_{i i^{*}}=1$, see also [25, 26]) we recover the relation (3.18), or, (3.4) directly from one of the bulk-boundary equations. As discussed in Section 3.5, from this relation we reconstruct the graph algebra.

In the diagonal case $\mathcal{E}=\mathcal{I}$, where $\psi_{a}^{j}=S_{a j}$, the relation (3.18) coincides with the Verlinde formula, i.e., the standard fusion algebra realised by its characters. On the other hand the Verlinde formula is known [14] to be recovered from the diagonal version of the other bulk-boundary equation, the Cardy-Lewellen equation (4.31) to which we now turn. This equation simplifies for $r=1$, leading to $\bar{k}=k^{*}, \bar{l}=l^{*}$. Using (4.43) and denoting $p_{i}(a)=\psi_{a}^{i} / \psi_{a}^{1}$ the equation (4.31) turns into

$$
p_{k}(a) p_{l}(a)=\sum_{j} M_{k l}^{j} p_{j}(a)
$$


where

$$
\begin{aligned}
M_{k l}{ }^{j} & =\sum_{t, \bar{t}} d_{\left(k, k^{*}\right)\left(l, l^{*}\right)}^{\left(j, j^{*} ; t \bar{t}\right.} \\
& :=\sqrt{\frac{d_{k} d_{l}}{d_{j}}} \sqrt{\frac{C_{j j^{*}}}{C_{k k^{*}} C_{l l^{*}}}} \sum_{t, \bar{t}} D_{\left(k, k^{*}\right)\left(l, l^{*}\right)}^{\left(j, j^{*}\right) t, \bar{t}} F_{j 1}\left[\begin{array}{cc}
k^{*} & k \\
l & l
\end{array}\right]_{\sigma_{13}(\bar{t}) t}^{\mathbf{1 1}},
\end{aligned}
$$

and $M_{k l}{ }^{j}=0$ if the corresponding Verlinde multiplicity $N_{k l}{ }^{j}$ vanishes. Alternatively, inverting (4.44),

$$
M_{k l}^{j}=\sum_{a \in \mathcal{V}} \frac{\psi_{a}^{k} \psi_{a}^{l} \psi_{a}^{j *}}{\psi_{a}^{1}} \quad k, l, j \in \mathcal{E} .
$$

Let us first look at the diagonal case in which according to (4.46) the constants $M_{k l}{ }^{j}$ coincide with the Verlinde fusion rule multiplicities $N_{k l}{ }^{j}$. This is confirmed also directly by the alternative expression (4.45) provided by equation (4.31) as we shall now show. In the diagonal case, denoting $D_{\left(k, k^{*}\right)\left(l, l^{*}\right)}^{\left(j, j^{*} ;, \bar{t}\right.}=C_{\left(k, k^{*}\right)\left(l, l^{*}\right)}^{\left(j, j^{*}\right), t \bar{t}}$, we can use the inverted equation (4.32) taken for $a=1$, a choice which trivialises all summations, since $n_{r 1}{ }^{1}=N_{r 1}{ }^{1}=\delta_{1 r}$, with the result (pointed out in the $\operatorname{sl}(2)$ case in 70 )

$$
\frac{{ }^{1} B_{j}^{1}}{{ }^{1} B_{k}^{1}{ }^{1} B_{l}^{1}} e^{i \pi\left(\triangle_{k}+\triangle_{l}-\triangle_{j}\right)} C_{\left(k, k^{*}\right)\left(l, l^{*}\right)}^{(j, j *), \bar{t}}=F_{1 j}\left[\begin{array}{cc}
k & l \\
k & l^{*}
\end{array}\right]_{\mathbf{1} \mathbf{1}}^{\sigma_{12}(\bar{t}) t} .
$$

Taking in particular $j=1$ (4.47) gives

$$
{ }^{1} B_{k}^{11} B_{k^{*}}^{1}=e^{2 \pi i \triangle_{k}} d_{k} C_{\left(k, k^{*}\right)\left(k^{*}, k\right)}^{(1,1)} .
$$

Comparing with (4.43) taken in the diagonal case we see that we can identify the undetermined constant $C_{i i^{*}}$ with the normalisation constant of the bulk 2-point function. We shall retain this identification of $C_{i i^{*}}$ in the non-diagonal cases (at the same level as the given diagonal case) which amounts to setting the relative 2-point normalisation to 1 . Combined with (4.35) and (4.43) the relation (4.47) leads to a symmetry of the fusing matrices analogous to the cyclic symmetry (4.18)

$$
F_{1 j}\left[\begin{array}{cc}
l & k \\
l & k^{*}
\end{array}\right]_{11}^{t_{1} t_{2}} d_{l}=F_{1 l}\left[\begin{array}{cc}
j & k^{*} \\
j & k
\end{array}\right]_{11}^{t_{2} t_{1}} d_{j}
$$

Inserting (4.47) back into (4.45) and using (4.49) reduces the sum over $\bar{t}$ to the standard pentagon identity specialised for some choice of the indices (cf. the analogous relation (4.38)). Finally we are left with the sum over the coupling index $t=t\left(\begin{array}{l}j \\ k l\end{array}\right)$, which reproduces the Verlinde multiplicity $N_{k l}{ }^{j}$ and completes the argument; alternatively the same conclusion is achieved using the simple choice of gauge (E.2).

Note that the relation (4.47), with (4.48) accounted for, is a linear version of the standard (quadratic) relation for the full plane diagonal OPE coefficients which results from locality of the (full) plane bulk fields 4-point functions, see Appendix E. In the $s l(2)$ case this identifies 
the OPE coefficients of the half- and full-plane diagonal bulk fields. The identification extends to the nondiagonal $s l(2)$ scalar OPE coefficients as can be seen generalising to 2point bulk correlators the computation of the limit $L / T \rightarrow \infty$ of the 1-point correlators in [11], leading to $(4.43)$.

In the general (non-diagonal) sl(2) cases characterised by a fixed level (central charge) we can express the fusing matrix in the r.h.s. of (4.45) in terms of that in the r.h.s. of (4.47). Using once again the $\operatorname{sl}(2)$ versions of the identities just described, we express it in terms of the diagonal OPE coefficients at the same level, obtaining for $N_{k l} l^{j}=1$

$$
M_{k l}^{j}=d_{(k, k)(l, l)}^{(j, j)}=D_{(k, k)(l, l)}^{(j, j)} / C_{(k, k)(l, l)}^{(j, j)} .
$$

The relative scalar OPE coefficients $d_{(k, k)(l, l)}^{(j, j)}$ have been computed for the $s l(2)$ WZW and the Virasoro (unitary) minimal models, see, e.g., [52] for an exhaustive list of references. Now using the expression for the eigenvectors (2.38), they can be computed as in (4.46) for all minimal models.

The matrices $\left(M_{k}\right)_{l}^{j}=M_{k l}{ }^{j}$ can be seen as a matrix realisation of an associative commutative algebra with identity, distinguished basis and an involution $*$. In (4.44) the algebra is realised by its 1-dimensional representations (characters) given by ratios of elements of the eigenvector matrix defining $n_{i}$. This algebra, traditionally called the "Pasquier algebra" ("M"-algebra), is dual in the sense of ref. [71] to the graph $\hat{N}$ - algebra considered in Section 3 but unlike its dual, its structure constants are not in general integral, but rather algebraic numbers. In the simplest $s l(2)$ case the squares $\left(M_{k l}{ }^{j}\right)^{2}$ of these constants are rational numbers for all $A-D-E$ cases; this rule persists for most of the $\operatorname{sl}(3)$ cases but is broken by two of the graphs $\mathcal{E}_{1}^{(12)}$ and $\mathcal{E}_{2}^{(12)}$ corresponding to the exceptional modular invariant at level $k+3=12$, see Appendix D. The type II $s l(2)$ cases, $D_{\text {odd }}$ and $E_{7}$ are again distinguished by the fact that the sign of some of the multiplicities $M_{k l}{ }^{j}$ is negative and this is a basis independent statement in the sense that there is no choice of basis to make all $M_{k l}^{j}$ non-negative, contrary to the Type I cases $D_{\text {even }}, E_{6}, E_{8}$, and this is a general feature of type II theories.

The formula (4.50) extends beyond the $s l(2)$ case for $(k, l, j)$ such that $N_{k l}{ }^{j}=1$, i.e., in cases with trivial Verlinde multiplicity the matrix elements $M_{k l}{ }^{j}$ provide the (relative) OPE coefficients $d_{\left(k, k^{*}\right)\left(l, l^{*}\right)}^{\left(j, j^{*}\right)}$. For non-trivial Verlinde multiplicities $N_{k l}{ }^{j}>1$ the relation between the constants $M_{k l}{ }^{j}$ (4.46) and the OPE coefficients is not so direct. Let us give a $s l(3)$ WZW example which illustrates the relation (4.45). There are three graphs found in [39] which correspond to the exceptional block-diagonal modular invariant at level $k+3=12$, see Appendix D, where these graphs are denoted by $\mathcal{E}_{i}^{(12)}, i=1,2,3$. One can pick up triplets of weights $(i, j, l)$ such that the Verlinde multiplicity of the diagonal $\operatorname{sl}(3)$ model at level $k+3=12$ is trivial, $N_{i j}{ }^{l}=1$ and check the values of the corresponding Pasquier algebra structure constants $M_{i j}{ }^{l}$ for each of the three graphs. The result is that, comparing in particular $\mathcal{E}_{1}^{(12)}$ and $\mathcal{E}_{3}^{(12)}$, there exist such triplets leading to different values of $M_{i j}{ }^{l}$ for the two graphs. Since for trivial Verlinde multiplicities the formula (4.45) gives a direct relation between the two types of constants, $M_{i j}^{l}=d_{\left(i, i^{*}\right)\left(j, j^{*}\right)}^{\left(l, l^{*}\right) ;}$, this result suggests that 
there are two different solutions for the bulk OPE coefficients in this case. Only one of these two non-diagonal solutions, namely the one which can be associated with the type I graph $\mathcal{E}_{1}^{(12)}$ was recovered in [53], exploiting a set of equations for the $M$-algebra structure constants. This set was derived from the bulk CFT locality equations assuming an additional (quadratic) constraint on the OPE coefficients in theories with an extended symmetry; some of its consequences were also reproduced in the abstract framework of [55, in particular the relation $n_{i 1}{ }^{a}=\operatorname{mult}_{a}(i)$ discussed in Section 3. Precisely this relation fails (and hence the assumptions on the OPE coefficients in [53]) for the graph $\mathcal{E}_{3}^{(12)}$, which otherwise satisfies all the requirements of type $\mathrm{I}$.

We conclude with a comment on the OPE coefficients. As discussed in [25, 26] one can relate in the limit $L / T \rightarrow \infty$ the correlators of the half- and full-plane bulk fields $\Phi_{I}^{H}(z, \bar{z})$ and $\Phi_{I^{\prime}}^{P}(\zeta, \bar{\zeta})$, looking at the two dual representations of the partition function with field insertions; in particular (4.43) was recovered in this way. Though this transformation needs to be elaborated for higher rank cases it seems reasonable to expect (and in agreement with (4.47)) that using the two choices of the automorphism $\Omega$, discussed in Section 2.1, we can identify in this way the OPE coefficients of the two bulk fields with either $I^{\prime}=I=(j, \bar{j})$ or $I^{\prime}=\left(j, \bar{j}^{*}\right)$.

$A$ bit of history: The algebra (4.44) defined through the eigenvectors of the $A-D-E$ Cartan matrices first appeared in the context of the $\operatorname{sl}(2) A-D-E$ lattice models proposed by Pasquier [50] a short time before the Verlinde fusion rule formula (the "A" algebra in the $\operatorname{sl}(2)$ case) was found. The interpretation in terms of a pair of dual $\mathrm{C}$ ('Character')-algebras was proposed in [51] in the discussion of the set of graphs found in [39] as a generalisation of the Dynkin diagrams associated with the modular invariants of $\operatorname{sl}(3) \mathrm{WZW}$ and minimal models. The fact that the relative scalar OPE coefficients $d_{(k, k)(l, l)}^{(j, j)}$ of all $A-D-E$ series of the $s l(2)$ WZW (or the subfamily of fields $(1, s)$ in unitary minimal models) coincide in a suitable basis with the Pasquier algebra (4.44) structure constants $M_{k l}{ }^{j}$ was the main result of [52]. It was established through a case by case check, supported by a lattice model derivation in which the same coefficients appear considering representations of the Temperley-Lieb algebra. CFT locality constraints resulting in formulae quite similar in spirit to (4.45) were furthermore exploited in [53], [54] as an ingredient in the construction of generalised Pasquier algebras and thus of new examples of graphs related to $\operatorname{sl}(n)$ modular invariants, extending the results in [39], [51]. The authors of [52], [53], [54] were however not aware of the parallel development of boundary CFT, and in particular of [11], [12], where the equation (4.31) first appeared. The importance of the algebra obtained from this equation at $r=1$ was recognised and stressed in [14], where a representative example of the $\operatorname{sl}(2) \mathrm{WZW} D_{\text {odd }}$ series was considered, for which the set $\mathcal{V}$ and the characters $\hat{\chi}_{i}(a)$ of the algebra were explicitly described. Presumably the authors of [14] were not aware of the general $A-D$ - $E$ result in [52]. In the same framework of boundary CFT the Pasquier algebra reappeared recently in a systematic study of orbifold theories, see [29] and references therein, under the name "(total) classifying algebra". 


\subsubsection{Relation to the Moore-Seiberg set of duality equations}

We have seen that the two Lewellen bulk-boundary equations (4.30) and (4.31) when restricted to some particular values of the indices $p$ in ${ }^{b} B_{(i, \bar{i})}^{p}$ become in some sense "dual" to each other, recovering the two dual $\mathrm{C}$-algebras, the graph and Pasquier algebras. These algebras are identical in the diagonal case, reproducing the Verlinde fusion algebra, which suggests that in this case the above two equations might be related.

On the other hand let us recall that the original derivation [34, 33] of the Verlinde formula relies on the use of one of the basic Moore-Seiberg duality relations, namely the equation resulting from the modular property of the two-point functions on the torus, see (E.7). It involves the fusing/braiding matrices $F$ or $B$ and the modular matrix $S_{i j}(p)$ (in general $\left.S_{i j}^{t^{\prime} t}(p)\right)$ for the 1-point functions $\chi_{j}^{(p)}(\tau, z)$ on the torus, the index $p$ standing for the (representation) label of the inserted CVOs $\left(\begin{array}{c}i \\ p i\end{array}\right)_{t, z^{\prime}}$ and $\left(\begin{array}{l}j \\ p j\end{array}\right)_{t, z}, \log z^{\prime}=(\log z) / \tau$, see [33.

The alert reader may have already noticed the full analogy between the Moore-Seiberg torus identity (E.7) and the second version (4.41) of equation (4.30). It suggests that the quantity taking over the role of the modular matrix $S(p)$ is the bulk-boundary reflection coefficient $B^{p}$. In the diagonal case this correspondence is precise, i.e., the two are identical up to a constant.

Indeed first note that equation (4.41), still considered in the general (non-diagonal) case, simplifies for $s=1$, that is $k=j^{*}, \bar{i}=i^{*}, \alpha^{\prime}=1$. Inserting in the first line the expression for the modular matrix $S_{i j}(p)$, see (E.8), and using in the third line (4.38), we obtain an expression for the modular matrix $S(p)$,

$$
{ }^{a} B_{i}^{1}\langle\mathbf{1}\rangle_{a} \frac{1}{F_{j^{*} 1}\left[\begin{array}{cc}
j & j^{*} \\
p & p
\end{array}\right]} \frac{S_{j i}\left(p^{*}\right)}{S_{i 1}}=\sum_{b, \beta}{ }^{b, \beta} B_{(i, i)}^{p}\langle\mathbf{1}\rangle_{b} \sum_{\delta}{ }^{(1)} F_{b j}\left[\begin{array}{cc}
p & j \\
b & a
\end{array}\right]_{\beta \delta}^{\delta} .
$$

Let us concentrate now on the diagonal case $\mathcal{I}=\mathcal{E}$. The sums in the r.h.s. of (4.51) can be reduced to one term choosing $a=1$ and using (4.12), since $n_{j 1}{ }^{b}=N_{j 1}{ }^{b}=\delta_{j b}, \delta=1$. Alternatively, one can take $a=1$ directly in the original equation (4.30) - the resulting (linear) formula for $B^{p}$ in terms of the fusing matrices $F$ (instead of the formula for its square derived in [11]) was first explicitly written down by Runkel [70]. More explicitly we have in the $s l(2)$ case,

$$
{ }^{j} B_{(i, i)}^{p}=\frac{{ }^{1} B_{i}^{1}}{d_{i} d_{j}} \frac{1}{F_{i 1}\left[\begin{array}{ll}
i & i \\
p & p
\end{array}\right]} \frac{S_{i j}(p)}{S_{11}}=\frac{{ }^{1} B_{i}^{1}}{d_{i} d_{j}} \frac{1}{F_{j 1}\left[\begin{array}{ll}
j & j \\
p & p
\end{array}\right]} \frac{S_{j i}(p)}{S_{11}} .
$$

Both (4.51) and (4.52) easily extend beyond the $s l(2)$ case. In particular restoring all coupling indices the latter formula reads in general

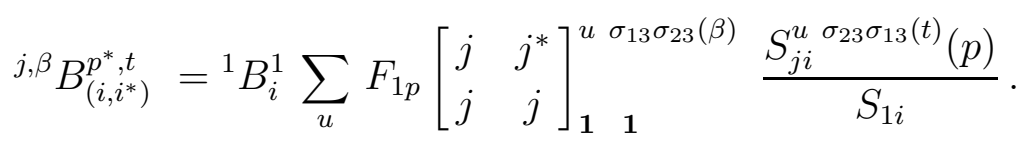


With the help of one of the consequences of the pentagon identity, (4.53) can be inverted and brought into a form analogous to that of (4.52).

The coincidence of the two seemingly very different quantities, the coefficients $B^{p}$ in the expansion (4.23) of the half-plane bulk field and the modular matrix $S(p)$ of the torus 1 -point blocks, is quite surprising and needs a better understanding. We were led to this observation trying to find a connection between the two duality schemes, the one of MooreSeiberg involving the torus, the other, of Cardy-Lewellen, involving the cylinder. Thus to bring equation (4.41), derived from the first of the Lewellen bulk-boundary equations (4.30), into a form identical to the original Moore-Seiberg torus duality relation we furthermore need to identify the three fusing matrices, ${ }^{(1)} F, F,{ }^{(3)} F$, i.e.,

$$
{ }^{(1)} F_{b p}\left[\begin{array}{ll}
k & j \\
a & c
\end{array}\right]_{\alpha \beta}^{\gamma t}=F_{b p}\left[\begin{array}{ll}
k & j \\
a & c
\end{array}\right]_{\alpha \beta}^{\gamma t}, \quad{ }^{(3)} F_{p b}\left[\begin{array}{ll}
c & j \\
a & k
\end{array}\right]_{\gamma^{\prime} t^{\prime}}^{\alpha^{\prime} \beta^{\prime}}=F_{p b}\left[\begin{array}{ll}
c & j \\
a & k
\end{array}\right]_{\gamma^{\prime} t^{\prime}}^{\alpha^{\prime} \beta^{\prime}} \text {. }
$$

This identification is consistent since in the diagonal case $\mathcal{I}=\mathcal{E}$ both mixed pentagon identities (4.29) and (4.37) then become the ordinary Moore-Seiberg pentagon identity; see also [70, where the identification of the boundary field OPE coefficients with the fusing matrices in the $\operatorname{sl}(2)$ case was first established by a more elaborate argument.

It is now straightforward to show that the first and second lines in (4.41) reproduce the two sides of the Moore-Seiberg identity (E.7). Taking into account (4.52) and (4.54) the second Lewellen bulk-boundary equation (4.31) is seen also to be a consequence of the first, i.e., of the same Moore-Seiberg torus duality relation. To show this, one has to insert in (4.31) the expression (4.47) for the OPE coefficients and to compare the equation with (4.41) with $p=1$, see also Appendix E.

We thus see that in the diagonal case the two basic bulk-boundary equations (4.30), (4.31) are not independent and are equivalent to one of the basic Moore-Seiberg duality relations. The third bulk-boundary equation (4.33), a more general version of (4.31), is an identity which involves again only the Moore-Seiberg duality matrices $F, B, S$, and thus can be expected, following the completeness argument of [33], to be derivable using the basic Moore-Seiberg duality relations. This in particular implies that any solution of the set of Moore-Seiberg (chiral) duality relations provides a solution of the diagonal boundary CFT equations.

Remark: Rewritten in terms of $S(p)$ the diagonal case Lewellen equation (4.31) can be also interpreted as a generalised Verlinde fusion formula with (non-integral) "multiplicities" $\mathcal{F}_{q k}^{l}$ given by some particular $F$ matrix elements. The matrices $\mathcal{F}_{q}$ are "diagonalised" by $S(p)$ with the usual eigenvalues $S_{q i}(1) / S_{1 i}(1)$. Because of this they realise another representation of the usual Verlinde algebra. This formula, which derives from the Moore-Seiberg torus duality identity, appears to have been already considered, following a different motivation, in [72].

We conclude this section with a few comments on the general non-diagonal cases. The Cardy-Lewellen boundary CFT can be looked at as a purely "chiral" alternative of the usual CFT approach in which we combine left and right chiral blocks imposing consistency 
conditions. It has its 'price' in that everything effectively "splits" - the set $\mathcal{I}$ is replaced by two "dual" sets $\mathcal{V}$ and $\mathcal{E}$ (for type I, while for type II we have to retain the whole $\mathcal{I}$ to describe nonscalar fields), there are two representations of the Verlinde fusion algebra and a related new fusion algebra (at least in type I cases); there are two types of "chiral vertex operators", new duality matrices, in particular a second fusing matrix ${ }^{(1)} F$ and its inverse, ${ }^{(3)} F$, along with the standard $F$, satisfying new duality relations, the mixed pentagon relations, generalising one of the basic genus zero polynomial identities; instead of one relation involving the modular matrix $S(p)$, there are two independent relations - the two bulk-boundary equations in which the role of $S(p)$ is taken over by the reflection coefficients $B^{p}$. It remains to find a consistent solution of the equations at least in the $s l(2)$ case. Some of the ingredients are already known and have been recalled above. In particular the solution for the $D$-series has just been obtained by Runkel 82.

\section{Conclusions and Outlook}

In this paper we have reexamined various aspects of boundary effects in RCFTs. We have in particular analyzed the consistency conditions that determine the allowed boundary states and fields and their characteristic data, OPE coefficients, etc. We have seen that boundary conditions are naturally associated with a graph, or a collection of graphs, whose spectral properties (eigenvalues) encode the diagonal spectrum of the bulk theory. This legitimates empirical observations made previously on the role of graphs in the classification of RCFTs. We have seen that the torus partition function may be fully reconstructed from the information contained in these graphs. We have seen also that in several cases $\left(\widehat{s l}(2), \widehat{s l}(N)_{1}\right.$ theories), this approach provides a substantially simpler route to the classification of RCFTs than the study of bulk properties (modular invariants...). We have finally seen that further important information about some boundary effects ( $g$-factors, boundary structure constants) is also encoded in the spectral properties (eigenvectors) of these graphs. The bottom line of this analysis is that a triplet of matrix algebras $\left(n_{j}, \hat{N}_{a}, M_{i}\right)$ plays a central role in the whole discussion. These algebraic structures have been also confirmed by the detailed analysis of the basic equations of the boundary field theory. In the diagonal case the triplet of algebras reduces to one, the Verlinde fusion algebra. Accordingly, we have seen that in this case the basic boundary CFT Lewellen equations can be identified with a set of genus 0 (the pentagon) and genus 1 duality identities of Moore-Seiberg. This leads to an identification of some of the basic notions in the two approaches, namely, the boundary fields OPE coefficients ${ }^{(1)} F$ and the bulk-boundary reflection coefficients $B^{p}$, with the chiral CFT fusing matrix $F$ and the modular matrix $S(p)$, respectively (see the text for precise formulae).

The more general representations $n_{i}$ of the Verlinde fusion algebra and the dual pair $\{\hat{N}, M\}$ of associative, commutative (semisimple) algebras have been introduced in earlier work on bulk (and later on boundary) conformal field theories, but it seems to us that the consistency of the whole scheme now appears in its full generality and that boundary 
RCFTs reveal these features in a simpler and more compelling way than in the bulk. In a loose sense, the boundary effects expose better the underlying chiral structure of the theory and its algebraic pattern. This should certainly not come as a surprise, as this is in the same spirit as the old connection between open and closed strings.

The study of a RCFT through its boundary conditions, its algebra triplet, etc, still requires a lot of work. The derivation of the Cardy equation relies on a technical assumption that has been only partially justified, namely the proper definition of unspecialized characters with linear independence and good modular properties for general chiral algebras. Also it would be good to have a better understanding of the completeness assumption: given a certain number of boundary conditions satisfying the Cardy equation, is it obvious that we may always supplement them into a complete set in the sense discussed in Section 2? Then many questions have been only partially treated: Justify in full generality the validity of expressions (3.11, 3.12) which have been established so far only for particular cases; understand better the nature and fusion rules of "twisted" block representations that appear in this discussion; set up a general scheme for the systematic classification of integer valued representations of fusion algebras; set up with more rigour the formalism of generalized chiral vertex operators, their fusing matrices and the ensuing duality equations as a consistent chiral approach, alternative to the Moore-Seiberg scheme, etc, such are some of the outstanding problems that are awaiting a proper treatment.

Also it remains to see how our discussion of boundary conditions must be generalized in theories where there is no choice of a common diagonalising matrix $\psi_{a}^{j}$ leading to $\hat{N}$ algebra with integer structure constants. In the approach of [55, 56], in which the numbers $\hat{N}$ are integers, one has to drop the axiom of commutativity, replacing this algebra by a noncommutative structure. In that respect, a better understanding of the relation of our work with other more abstract approaches - Ocneanu theory of subfactors, weak Hopf algebras — would be most profitable.

Directions for future work also include the discussion of other cases: rational or irrational theories at $c=1, c=2$, or $\mathcal{N}=2$ superconformal theories are particularly important cases in view of their physical applications to condensed matter or to string theory. The generalization to other types of twisted boundary conditions along the cycle of the cylinder, as examined recently in [83], might constitute another useful approach. Finally the parallel discussion of these boundary conditions and algebraic structures in lattice models should be extremely instructive and will be the object of a forthcoming publication.

\section{Appendix A: The Cardy Equation}

In this Appendix, we rederive the Cardy equation (Section 2.2) in the presence of sources, which have the effect of introducing unspecialized characters in the partition function. We restrict to a conformal field theory with a current algebra. Let $\left\{J^{\alpha}\right\}$ denote the generators in the Cartan subalgebra, and $\nu_{\alpha}$ be "charges" coupled to them. We consider the theory on 
the cylinder $L \times T$ of Section 2.2, call $w=u+i v$ the local variable, $0 \leq v \leq L$, $u$ periodic of period $T$, and modify the energy-momentum tensor $T(w)$ into

$$
\begin{aligned}
& T^{\prime}(w)=T(w)-\frac{2 i \pi}{T} \sum_{a} \nu_{\alpha} J^{\alpha}(w)-\frac{k}{2} \sum_{\alpha}\left(\frac{2 \pi \nu_{\alpha}}{T}\right)^{2} \\
& \bar{T}^{\prime}(\bar{w})=\bar{T}(\bar{w})-\frac{2 i \pi}{T} \sum_{\alpha} \nu_{\alpha} \bar{J}^{\alpha}(\bar{w})-\frac{k}{2} \sum_{\alpha}\left(\frac{2 \pi \nu_{\alpha}}{T}\right)^{2} .
\end{aligned}
$$

As an elementary calculation shows, the last term is dictated by the requirement that $T^{\prime}$ satisfies the conventional OPE of an energy momentum tensor. The central charge is not affected by the additional terms.

One then computes the evolution operators in the two channels of Section 2.2, see Figure 11. For the cylinder, mapped to the plane by $\zeta=e^{-2 \pi i w / T}$, the Hamiltonian reads

$$
\begin{aligned}
H^{\mathrm{cyl}} & =\frac{1}{2 \pi} \int_{0}^{-T} d u\left(T^{\prime}(w)+\bar{T}^{\prime}(\bar{w})\right)=\frac{1}{2 \pi} \oint d w\left(T^{\prime}(w)+\bar{T}^{\prime}(\bar{w})\right) \\
& =\left(\frac{2 \pi}{T}\right)\left(L_{0}^{(\mathrm{P})}+\bar{L}_{0}^{(\mathrm{P})}-\frac{c}{12}+\sum_{\alpha} \nu_{\alpha}\left(J_{0}^{(\mathrm{P}) \alpha}-\bar{J}_{0}^{(\mathrm{P}) \alpha}\right)\right)
\end{aligned}
$$

Note that the additional term in (A.1) and (A.2) has not contributed to the integral over a closed cycle. Taking into account the fact that on boundary states $L_{0}^{(\mathrm{P})}=\bar{L}_{0}^{(\mathrm{P})}$ and $J_{0}^{(\mathrm{P}) \alpha}=-\bar{J}_{0}^{(\mathrm{P}) \alpha}$, we find that the first expression of the partition function reads

$$
Z_{b \mid a}=\left\langle b\left|e^{-\frac{4 \pi L}{T}\left(L_{0}-\frac{c}{24}+\sum_{\alpha} \nu_{\alpha} J_{0}^{\alpha}\right)}\right| a\right\rangle=\sum_{j \in \mathcal{E}} \psi_{a}^{j}\left(\psi_{b}^{j}\right)^{*} \frac{\chi_{j}(\tilde{q}, \nu \tilde{\tau})}{S_{j 1}}
$$

where we have defined

$$
\chi_{j}(q, z):=\operatorname{tr} \mathcal{V}_{j} q^{L_{0}-\frac{c}{24}} e^{2 \pi i \sum_{\alpha} z_{\alpha} J_{0}^{\alpha}}
$$

and as above $\tilde{q}=e^{-4 \pi \frac{L}{T}}$, hence $\tilde{\tau}=2 i \frac{L}{T}$.

In the other channel, the time evolution on the strip is described by the Hamiltonian

$$
H_{b a}=\left(\int_{0}^{i L} \frac{d w}{2 \pi i} T^{\prime}(w)+\int_{0}^{-i L} \frac{d \bar{w}}{2 \pi i} \bar{T}^{\prime}(\bar{w})\right)
$$

and upon mapping on the upper half plane $H$ by $z=e^{\pi \frac{w}{L}}$, we find

$$
H_{b a}=\frac{\pi}{L}\left(L_{0}^{(H)}-\frac{c}{24}\right)-\frac{2 i \pi}{T} \sum_{\alpha} \nu_{\alpha} J_{0}^{H \alpha}-\frac{L}{2 \pi} k \sum_{\alpha}\left(\frac{2 \pi \nu_{\alpha}}{T}\right)^{2}
$$

where now the additional piece in (A.1) contributes the last term. Since the theory with energy momentum tensor (A.1) and (A.2) has still the same operator content and multiplicities $n_{i^{*} a}{ }^{b}=n_{i b}{ }^{a}$ as before, we may write

$$
Z_{b \mid a}=\operatorname{tr} e^{-T H_{b a}}=e^{2 \pi k \frac{L}{T} \sum_{\alpha} \nu_{\alpha}^{2}} \sum_{i} n_{i^{*} a}{ }^{b} \chi_{i}(q, \nu)
$$


with $q=e^{-\pi \frac{T}{L}}$. We then use the modular transformation of unspecialized characters (see 38], page 264):

$$
\chi_{i}(q, \nu)=e^{2 i \pi k \sum \frac{\tilde{z}_{\alpha}^{2}}{2 \tilde{\tau}}} \sum_{j} S_{i j} \chi_{j}(\tilde{q}, \nu \tilde{\tau})
$$

together with the linear independence of the $\chi_{i}(q, z)$ to conclude that $(2.16)$ is indeed true in full generality.

\section{Appendix B: $A-D-E$ Diagrams and Intertwiners}

In this Appendix we establish notations on $A-D-E$ Dynkin diagrams and on the associated intertwiners.

Let $G$ be a Dynkin diagram of the $A-D-E$ type with Coxeter number $g$. It has $n$ nodes that may be coloured with two colours, i.e. its $n \times n$ adjacency matrix $G_{a b}$ connects only nodes of different colours. This matrix is symmetric and it may thus be diagonalized in an orthonormal basis. We call this orthonormal basis $\psi_{a}^{m}$, it is labelled by the node $a$ and the exponent $m$ (see Figure 2 and Table 1). Hence

$$
\sum_{b} G_{a}{ }^{b} \psi_{b}^{s^{\prime}}=2 \cos \frac{\pi s^{\prime}}{g} \psi_{a}^{s^{\prime}} .
$$

The $\psi$ 's satisfy orthonormality conditions, namely

$$
\begin{aligned}
\sum_{a} \psi_{a}^{s^{\prime}} \psi_{a}^{s^{\prime \prime} *} & =\delta_{s^{\prime} s^{\prime \prime}} \quad s^{\prime}, s^{\prime \prime} \in \operatorname{Exp}(G) \\
\sum_{s^{\prime} \in \operatorname{Exp}} \psi_{a}^{s^{\prime}} \psi_{b}^{s^{\prime} *} & =\delta_{a b}
\end{aligned}
$$

Because of the 2-colourability of $G$, one may attach a $\mathbb{Z}_{2}$ grading $\tau$ to each node $a$. One proves that if $s^{\prime}$ is an exponent, so is $\sigma\left(s^{\prime}\right)=g-s^{\prime}$ and the $\psi$ 's may be chosen to satisfy

$$
\psi_{a}^{\sigma\left(s^{\prime}\right)}=(-1)^{\tau(a)} \psi_{a}^{s^{\prime}} .
$$

Moreover all graphs having even exponents, v.i.z. the $A, D_{\text {odd }}$ and $E_{6}$ diagrams, have a $\mathbb{Z}_{2}$ automorphism $\gamma$ acting on their nodes and preserving their adjacency matrix (i.e. $G_{a}{ }^{b}=G_{\gamma(a)}{ }^{\gamma(b)}$, this is the natural $\mathbb{Z}_{2}$ symmetry of these graphs) such that

$$
\psi_{\gamma(a)}^{s^{\prime}}=(-1)^{\tau\left(s^{\prime}\right)} \psi_{a}^{s^{\prime}} .
$$

Finally, one may find in the graph $G$ a distinguished node labelled $a=1$ such that $\psi_{1}^{m}>0$ for all $m$. This special node is typically an extremal node, i.e. the end of a branch for the $A-D-E$ graphs; this is generally the end of a long leg, but for the $D_{\text {odd }}$ graphs, for which we must choose 1 as the end point of one of the two short legs.

We list hereafter the explicit expressions of eigenvectors of the various Dynkin diagrams. 
The $D_{\frac{g}{2}+1}$ series are the simplest examples of orbifold models. Their fundamental graphs can be obtained by folding the $A_{g-1}$ Dynkin diagram so that the nodes $a_{i}=a_{g-i} \in D_{\frac{g}{2}+1}, i=$ $1,2,3, \ldots, \frac{g}{2}-1$ are identified with the orbit $\{i\}$ of $i$ under the $\mathbb{Z}_{2}$ automorphism $\sigma, \sigma(i)=g-$ $i$, while the fixed point $i=g / 2$ is resolved into two points $a_{\frac{g}{2}, \pm}$ on the graph, denoted $n, n-1$ in Figure 2. This implies for the adjacency matrix elements $G_{a_{i} a_{j}}=A_{i j}+A_{i \sigma(j)}=A_{i j}$, for $i, j \neq \frac{g}{2}$, and $G_{a \frac{g}{2}, \pm} a_{j}=A_{\frac{g}{2} j}$ and allows us to determine the eigenvectors $\psi_{a}^{j}$ of $G$ in terms of the eigenvectors $S_{i j}$ of the $A$ adjacency matrix. To simplify notation we shall use sometimes $\psi_{i}^{j}=\psi_{a_{i}}^{j}$.

\section{Eigenvectors of the $D_{2 l}$ adjacency matrix}

$$
\begin{aligned}
\psi_{i}^{j} & =\sqrt{2} S_{i j}, \quad i, j \neq \frac{g}{2}, \quad \psi_{i}^{\left(\frac{g}{2}, \pm\right)}=S_{i \frac{g}{2}}, \quad i \neq \frac{g}{2} ; \\
\psi_{\frac{g}{2}, \pm}^{j} & =\frac{S_{\frac{g}{2}} j}{\sqrt{2}}, \quad j \neq \frac{g}{2} ; \quad \psi_{\frac{g}{2}, \epsilon}^{\left(\frac{g}{2}, \epsilon^{\prime}\right)}=S_{\left\{\left(\frac{g}{2}, \epsilon\right)\right\}\left\{\left(\frac{g}{2}, \epsilon^{\prime}\right)\right\}}^{e x t}=\frac{1}{2}\left(S_{\frac{g}{2} \frac{g}{2}}+\epsilon \epsilon^{\prime} i \sqrt{(-1)^{l}}\right) .
\end{aligned}
$$

For $i$-odd the orbits $\{i\}$ belong to $\mathcal{I}^{e x t}$ and can be identified with the subset $T=\left\{a_{i}, i=\right.$ $1,3, \ldots, \frac{g}{2}-2, a_{\left.\frac{g}{2}, \pm\right\}}$. The matrix $S_{\{i\}\{j\}}^{e x t}$ is the extended theory modular matrix. The expressions (B.6) can be rewritten in the compact form

$$
\psi_{a}^{j}=S_{a\{j\}} \sqrt{\frac{S_{1 j}}{S_{\{1\}\{j\}}^{e x t}}},
$$

where $S_{a\{j\}}$ is a rectangular matrix coinciding for $a \in T$ with $S_{a\{j\}}^{e x t}$, while for $a=a_{i} \notin T$, $S_{a_{i}\left\{\left(\frac{g}{2}, \pm\right)\right\}}=S_{i \frac{g}{2}}=0$ and $S_{a_{i}\{j\}}=\sum_{l \in\{i\}} S_{l j}=2 S_{i j}$ for $j \neq g / 2$.

\section{Eigenvectors of the $D_{2 l+1}$ adjacency matrix}

$$
\begin{aligned}
\psi_{a_{i}}^{j} & =(-1)^{\frac{j-1}{2}} \sqrt{2} S_{i j}, \quad i, j \neq \frac{g}{2} ; \quad \psi_{a_{i}}^{\frac{g}{2}}=0, \quad i \neq \frac{g}{2} \\
\psi_{\frac{g}{2}, \pm}^{j} & =(-1)^{\frac{j-1}{2}} \frac{1}{\sqrt{2}} S_{\frac{g}{2} j}=\frac{1}{\sqrt{g}}, \quad j \neq \frac{g}{2} ; \quad \psi_{\frac{g}{2}, \pm}^{\frac{g}{2}}= \pm \frac{1}{\sqrt{2}} .
\end{aligned}
$$

The identity node is chosen to coincide with one of the 'fork' nodes $1=a_{\frac{g}{2},+}$ (denoted by $L$ in Figure 2) so that the dual Perron-Frobenius eigenvector $\psi_{1}^{j}=\psi_{\frac{g}{2},+}^{j}$ has positive entries (while $\psi_{a_{1}}^{\frac{g}{2}}=0$ ). The 'fundamental' node $f$ is identified with $\frac{g}{2}-1$, i.e., $G=\hat{N}_{\frac{g}{2}-1}$. Also $a^{*}=a$ for all $a$, while $\gamma\left(a_{\frac{g}{2},+}\right)=a_{\frac{g}{2},-}$.

Next we display the eigenvectors of the exceptional $E_{r}$ Dynkin diagrams as a matrix $\left\{\psi_{a}^{j}\right\}$, with the row index a running over the nodes, following the numbering of Figure 2, 
and the column index $j$ over the exponents in the same order as in Table 1 . There too, $S_{i j}$ denote the eigenvectors of the diagonal graph adjacency matrix $A$ with the same Coxeter number.

\section{Eigenvectors of the $E_{6}$ adjacency matrix}

$$
\left(\psi_{a}^{j}\right)=\left(\begin{array}{cccccc}
a & \frac{1}{2} & b & b & \frac{1}{2} & a \\
b & \frac{1}{2} & a & -a & -\frac{1}{2} & -b \\
c & 0 & -d & -d & 0 & c \\
b & -\frac{1}{2} & a & -a & \frac{1}{2} & -b \\
a & -\frac{1}{2} & b & b & -\frac{1}{2} & a \\
d & 0 & -c & c & 0 & -d
\end{array}\right)
$$

where $a=\frac{1}{2} \sqrt{\frac{3-\sqrt{3}}{6}}, b=\frac{1}{2} \sqrt{\frac{3+\sqrt{3}}{6}}, c=\frac{1}{2} \sqrt{\frac{3+\sqrt{3}}{3}}, d=\frac{1}{2} \sqrt{\frac{3-\sqrt{3}}{3}}$ are determined from $\psi_{1}^{i}=$ $\sqrt{S_{1 i} \sum_{j \in \rho} S_{j i}}, \psi_{6}^{i}=\left(S_{4 i}+S_{8 i}\right) \sqrt{\sum_{j \in \rho}^{S_{1 i} S_{j i}}}$ for $i \in \mathcal{E}, \rho=\{1,7\}$.

\section{Eigenvectors of the $E_{7}$ adjacency matrix}

$$
\left(\psi_{a}^{j}\right)=\left(\begin{array}{ccccccc}
a & c & b & \frac{1}{\sqrt{3}} & b & c & a \\
e & f & d & 0 & -d & -f & -e \\
c & b & -a & -\frac{1}{\sqrt{3}} & -a & b & c \\
f & -d & -e & 0 & e & d & -f \\
\frac{1}{\sqrt{6}} & -\frac{1}{\sqrt{6}} & \frac{1}{\sqrt{6}} & 0 & \frac{1}{\sqrt{6}} & -\frac{1}{\sqrt{6}} & \frac{1}{\sqrt{6}} \\
d & -e & f & 0 & -f & e & -d \\
b & -a & -c & \frac{1}{\sqrt{3}} & -c & -a & b
\end{array}\right)
$$

where $a, b, c, d, e, f$ are determined from $\psi_{1}^{j}=\sqrt{S_{1 j} \sum_{i \in \rho} S_{i j}}$ where $\rho=\{1,9,17\}$, and $\psi_{2}^{j}=\frac{S_{2 j}}{S_{1 j}} \psi_{1}^{j}$ (The values in the 5'th row come from $\psi_{5}^{j}=\sqrt{2} S_{6 j}$ for $j=1,5,7$.) Explicitly,

$$
\begin{array}{ll}
a=\left[18+12 \sqrt{3} \cos \frac{\pi}{18}\right]^{-\frac{1}{2}}, & d=\left[12\left(1+\cos \frac{\pi}{9}\right)\right]^{-\frac{1}{2}} \\
b=\left[18+12 \sqrt{3} \cos \frac{11 \pi}{18}\right]^{-\frac{1}{2}} & e=\left[12\left(1+\cos \frac{5 \pi}{9}\right)\right]^{-\frac{1}{2}} \\
c=\left[18+12 \sqrt{3} \cos \frac{13 \pi}{18}\right]^{-\frac{1}{2}}, & f=\left[12\left(1+\cos \frac{7 \pi}{9}\right)\right]^{-\frac{1}{2}} .
\end{array}
$$




\section{Eigenvectors of the $E_{8}$ adjacency matrix}

$$
\left(\psi_{a}^{j}\right)=\left(\begin{array}{cccccccc}
a & f & c & d & d & c & f & a \\
b & e & h & g & -g & -h & -e & -b \\
c & d & -a & -f & -f & -a & d & c \\
d & a & -f & -c & c & f & -a & -d \\
e & -h & -g & b & b & -g & -h & e \\
f & -c & d & -a & a & -d & c & -f \\
g & -b & e & -h & -h & e & -b & g \\
h & -g & -b & e & -e & b & g & -h
\end{array}\right)
$$

where $a, b, c, d, e, f, g, h$ are determined from $\psi_{1}^{j}=\sqrt{S_{1 j} \sum_{i \in \rho} S_{i j}}$ and $\psi_{2}^{j}=\frac{S_{2 j}}{S_{1 j}} \psi_{1}^{j}$ for $\mathrm{J} \in \mathcal{E}, \rho=\{1,11,19,29\}$. Explicitly

$$
\begin{array}{ll}
a=\left[\frac{15(3+\sqrt{5})+\sqrt{15(130+58 \sqrt{5})}}{2}\right]^{-\frac{1}{2}} & b=[15+\sqrt{75-30 \sqrt{5}}]^{-\frac{1}{2}} \\
c=\left[\frac{15(3+\sqrt{5})-\sqrt{15(130+58 \sqrt{5})}}{2}\right]^{-\frac{1}{2}} & e=[15-\sqrt{75+30 \sqrt{5}}]^{-\frac{1}{2}} \\
d=\left[\frac{15(3-\sqrt{5})-\sqrt{15(130-58 \sqrt{5})}}{2}\right]^{-\frac{1}{2}} & g=[15+\sqrt{75+30 \sqrt{5}}]^{-\frac{1}{2}} \\
f=\left[\frac{15(3-\sqrt{5})+\sqrt{15(130-58 \sqrt{5})}}{2}\right]^{-\frac{1}{2}} & h=[15-\sqrt{75-30 \sqrt{5}}]^{-\frac{1}{2}} .
\end{array}
$$

To such a graph $G$, one then attaches matrices $V_{i}$ as follows. The case of reference is the $A_{g-1}$ diagram of same Coxeter number $g$ as $G$. For this $A$ graph, both the nodes and the exponents take all integer values in $\{1, \cdots g-1\}$. The $\psi$ 's are then nothing other than the entries of the (symmetric, unitary) matrix $S$ of modular transformations of characters of the affine algebra $\widehat{s l}_{2}$ at level $g-2$

$$
\psi_{i}^{(A) i^{\prime}}=S_{i i^{\prime}}=\sqrt{\frac{2}{g}} \sin \frac{\pi i i^{\prime}}{g},
$$

in terms of which the fusion coefficients $N_{i_{1} i_{2}}{ }^{{ }^{3}}$ may be expressed through Verlinde formula. Note also that

$$
N_{g-i i_{1}}{ }^{g-i_{2}}=N_{i i_{1}}{ }^{i_{2}}
$$

because of property $(\mathbb{B} .4)$ applied to $\psi^{(A)}=\hat{S}$.

We now return to the graph $G$ of Coxeter number $g$. The fused adjacency matrices $V_{i}$ with $i=1, \ldots, g-1$ are $n \times n$ matrices defined recursively by the $\operatorname{sl}(2)$ fusion algebra

$$
V_{i}=V_{2} V_{i-1}-V_{i-2} \quad 2<i \leq g
$$

and subject to the initial conditions $V_{1}=I$ and $V_{2}=G$. (One may see that $V_{g}=0$ ). The matrices $V_{i}$ are symmetric and mutually commuting with entries given by a Verlinde-type formula

$$
V_{i a}{ }^{b}=\left(V_{i}\right)_{a}{ }^{b}=\sum_{m \in \operatorname{Exp}(G)} \frac{S_{i m}}{S_{1 m}} \psi_{a}^{m} \psi_{b}^{m *}
$$


Regarded as $(g-1) \times n$ rectangular matrices, for $a$ fixed, the $V_{i a}{ }^{b}$ intertwine the $A$ and $G$ adjacency matrices

$$
\sum_{i^{\prime}} A_{i}^{i^{\prime}} V_{i^{\prime} a}^{b}=\sum_{b^{\prime}} V_{i a}{ }^{b^{\prime}} G_{b^{\prime}}{ }^{b}
$$

Regarded as $n \times n$ matrices, the $V_{i}$ satisfy not only their defining relation (B.16) but also the whole $\widehat{s l}(2)$ fusion algebra (2.22)

$$
V_{i_{1}} V_{i_{2}}=\sum_{i_{3}} N_{i_{1} i_{2}}^{{ }^{i}} V_{i_{3}}
$$

From their recursive definition and initial conditions, it follows that the entries of the $V$ matrices are integers. What is not obvious is that these entries are non-negative integers. This follows either from a direct inspection or from an elegant group theoretic argument due to Dorey [74]. We refer the reader to [39, 48] for the explicit expressions of these intertwiners. As a consequence of the existence of the automorphism $\gamma$ defined above $(\mathbb{B} .5)$, a $\mathbb{Z}_{2}$ symmetry on $A, D_{\text {odd }}, E_{6}$ graphs and the identity for $D_{\text {even }}, E_{7}, E_{8}$, one has

$$
V_{g-s a}^{\gamma(b)}=V_{s a}^{b}
$$

Using (B.16) and (3.4) (i.e. in the notations of this Appendix, $V_{i} \hat{N}_{a}=\sum_{b} V_{i a}{ }^{b} \hat{N}_{b}$ ) one can express the graph algebra $\hat{N}_{a}$ matrices for all but the $D_{\text {even }}$ cases as polynomials of $V$ 's with integer coefficients This in particular ensures that they have integer matrix elements. For $D_{\text {even }}$ one of the extended fusion algebra generators $N_{\left\{\frac{g}{2}, \pm\right\}}^{e x t}$ has to be added since $V_{\frac{g}{2}}=N_{\left\{\frac{g}{2},+\right\}}^{e x t}+N_{\left\{\frac{g}{2},-\right\}}^{e x t}$ while $N_{i}=V_{i}=V_{g-i}$ for $i=1,2, \ldots, \frac{g}{2}-1$. For the three exceptional cases $E_{r}$ we have $N_{i}=V_{i}, i=1,2, \ldots r-3, N_{r-1}=V_{r-1}-V_{r-3}, N_{r-2}=V_{r}-V_{r-4}$, $N_{r}=V_{r-2}+V_{r-4}-V_{r}$, which translates into relations between the eigenvalues and given $\psi_{1}^{j}$ allows to express any $\psi_{a}^{j}$ in terms of the modular matrix $S$ elements.

Over recent years, these matrices have made repeated appearances in a variety of problems. Originally introduced in the discussion of local height probabilities in lattice models [50] and of boundary partition functions [9, 39] (see below), they have also appeared in the following contexts:

(i). The "cells" or intertwiners of Boltzmann weights of height models [39, 48].

(ii). The decomposition of the representation of the Temperley-Lieb algebra on the space of paths from $a$ to $b$ on graph $G$ onto the irreducible ones on the paths from 1 to $s$ on graph $A_{g-1}$ 49 according to $R_{a}^{(G) b}=\oplus_{s} V_{s a}{ }^{b} R_{1}^{(A)}{ }_{s}$.

(iii). The counting of "essential paths" on graphs [73]; see also recent mathematical work by $\mathrm{Xu}$, Böckenhauer and Evans [55, 56].

(iv). The expression of the blocks of the partition function (1.2) as (3.11, 3.12), see Section 3.3. 
(v). The $s l(2)$ intertwiners appear in the computation of the multiplicities $m_{s^{\prime}}^{b}$ of an irreducible representation $b$ of the finite group, associated with $G$ in the McKay correspondence [75, 76, in the $S U(2)$ representations of dimension $s^{\prime}$ 84. Namely the coefficients of the Kostant polynomials in the generating function $F_{b}$ of these multiplicities are given for a non trivial $b$ by $\sum_{c} G_{0 c} V_{s c}^{b}$, where $G_{a b}$ is the adjacency matrix of the affine Dynkin diagram and $a=0$ is the affine node deleted in passing from the affine Dynkin diagram to the ordinary one. The proof of this fact is reduced to the recursive relation (B.16).

(vi). These same entries seem to appear ubiquituously in the description of $S$-matrices of affine Toda theories [77] and in the description of the excitation spectrum of integrable lattice models [78, 79, 80].

\section{Appendix C: Uniqueness of the Boundary Conditions of Minimal Models}

\section{C.1. Matrices with spectrum $\gamma<2$}

We first recall general results on symmetric matrices with non-negative integer entries and with eigenvalues between -2 and 2 .

It is a standard result that symmetric matrices with non-negative integer entries and eigenvalues $\gamma \in]-2,2[$ may be classified. A lemma of Kronecker asserts that the eigenvalues are of the form $2 \cos \frac{p_{i} \pi}{h_{i}}$ for integers $p_{i}$ and $h_{i}$ and for the largest one(s), $p=1$. One may regard any such matrix as the adjacency matrix of a graph. Irreducible matrices correspond to connected graphs, and by an abuse of language one may call a matrix bicolourable if the graph has that property. One proves 40] that any irreducible bicolourable symmetric matrix with spectrum in ] $-2,2$ [ is the adjacency matrix of one of the simply laced Dynkin diagrams of type $A-D-E$.

If one relaxes the assumption of bicolourability, with any symmetric non-bicolourable irreducible matrix $G$ one may associate a bicolourable symmetric matrix with a block form $G^{\prime}=\left(\begin{array}{cc}0 & G \\ G & 0\end{array}\right)$. The corresponding graph is irreducible and has a $\mathbb{Z}_{2}$ symmetry that exchanges the two colours. Any eigenvalue $\gamma$ of $G$ gives rise to two eigenvalues $\pm \gamma$ for $G^{\prime}$ and one thus concludes that $G^{\prime}$ is of $A-D$-E type, and its irreducibility forces $G^{\prime}=A_{2 p}$. Its $\mathbb{Z}_{2}$ quotient $G$ is what we call the tadpole graph $T_{p}=A_{2 p} / \mathbb{Z}_{2}$.

Finally if one relaxes the assumption of irreducibility, one concludes that any matrix (with non-negative entries and spectrum between -2 and 2) is the direct sum of $A-D-E$ or tadpole graphs

$$
G=\oplus G_{i} \quad G_{i} \text { of } A-D-E \text { or tadpole type }
$$

and this decomposition is unique, up to the permutation of factors. The uniqueness may 
be easily proved by induction on the number of terms or on the dimension of the matrix: Given a matrix $G$, one first identifies its largest eigenvalue, of the form $\gamma_{1}=2 \cos \frac{\pi}{h_{1}}$. By the previous statement, there is an $A-D-E$ or tadpole graph $G_{1}$ with Coxeter number $h_{1}$ and exponents $m_{i}$, such that all its eigenvalues $2 \cos \frac{m_{j}}{h_{1}}$ appear in the spectrum of $G$. Thus $G=G_{1} \oplus G^{\prime \prime}$, and one may apply on $G^{\prime \prime}$ the induction hypothesis. If $\gamma_{1}$ has multiplicity 1 , this suffices to establish the uniqueness of the decomposition (up to permutations), while the case where $\gamma_{1}$ has non-trivial multiplicity is also easily dealt with. The uniqueness of this decomposition implies a property used several times in the text, namely that the spectrum (between -2 and 2) determines the form of the matrix up to a permutation of its rows and columns.

\section{C.2. Representatives of $n_{12}$ and $n_{21}$}

We now return to minimal models.

\section{Explicit form of $n_{12}$ and $n_{21}$}

It is convenient to work in a basis different from that used in (2.38). In the basis $r_{1}=1, \cdots, p$,

$a \in G$, the second term in (2.33) does not contribute to $n_{12}$ since $N_{2 p r_{1}}{ }^{r_{2}}=\delta_{r_{1}, 2 p+1-r_{2}}=0$ for $1 \leq r_{1}, r_{2} \leq p$. Thus $n_{12}=I_{p} \otimes V_{2}=I_{p} \otimes G$, where $I_{p}$ is the $p$ dimensional unit matrix

$$
n_{12}=I_{p} \otimes G=\left(\begin{array}{llll}
G & & & \\
& G & & \\
& & \ddots & \\
& & & G
\end{array}\right) \text {. }
$$

As for $n_{21}$, in the same basis, the second term of (2.33) receives a contribution only from $r_{1}=$ $r_{2}=p$, namely $\left(N_{2 p-1}\right)_{p}{ }^{p}=1$, while $V_{g-1}=\Gamma$, the matrix that realizes the automorphism $\gamma$ :

$$
\Gamma_{a}^{b}=\delta_{a \gamma(b)}
$$

Thus one finds that

$$
n_{21}=\left(\begin{array}{cccc}
0 & I_{n} & & \\
I_{n} & 0 & I_{n} & \\
& \ddots & \ddots & I_{n} \\
& & I_{n} & \Gamma
\end{array}\right) .
$$

After conjugation by a block-diagonal matrix with $\Gamma$ and $I_{n}$ in alternating positions, which leaves the form (C.1) of $n_{12}$ unchanged, $n_{21}$ may be recast in the form

$$
n_{21}=\left(\begin{array}{cccc}
0 & \Gamma & & \\
\Gamma & 0 & \Gamma & \\
& \ddots & \ddots & \Gamma \\
& & \Gamma & \Gamma
\end{array}\right)=T_{p} \otimes \Gamma,
$$

in terms of the tadpole $T_{p}$ adjacency matrix. All the other $n_{r s}$ are obtained as universal polynomials of the two matrices $n_{12}$ and $n_{21}$. 


\section{Uniqueness of the form of $n_{12}$ and $n_{21}$}

Conversely, suppose we only know that the representation $n_{r s}$ has a spectrum specified by the set of exponents $\mathcal{E}$. We want to prove that there exists a basis in which $n_{12}$ and $n_{21}$ take the forms (C.1) and (C.3).

We first make use of the property that the set $\operatorname{Exp}(G)$ is stable modulo the Coxeter number $g$ of $G$ under multiplication by any integer coprime to $g$ and is also stable under the reflection $s \rightarrow g-s$. We then find that the spectrum of $n_{12}$ is made of $p$ copies of $\operatorname{Exp}(G)$. As explained above in C.1, this implies that in some basis

$$
n_{12}=I_{p} \otimes G
$$

For the other generator $n_{21}$, one observes first that the set of numbers that appear in (2.31), namely $\left\{2 \cos \frac{\pi g r^{\prime}}{2 p+1}\right\}, r^{\prime}=1,3 \cdots, 2 p-1$, is simply the set $\left\{(-1)^{g+1} 2 \cos \frac{\pi r^{\prime \prime}}{2 p+1}\right\}$, $r^{\prime \prime}=1,3, \cdots, 2 p-1$, which is $(-1)^{g+1}$ times the spectrum of the tadpole $T_{p}$. According to (2.31), this has to be multiplied by $(-1)^{s^{\prime}}$, as $s^{\prime}$ runs over the exponents of $G$. Thus if $g$ is even (which is the general case except when $G=A_{2 l}$ ) the spectrum of $n_{21}$ is made of as many copies of that of $T_{p}$ (resp. $-T_{p}$ ) as there are odd (resp. even) exponents in $G$. For $A_{2 l}$ which has as many even as odd exponents, the same conclusion is still correct! Finally one notices that these signs are just the eigenvalues of the $\Gamma$ matrix, and one thus concludes that

$$
n_{21} \sim T_{p} \otimes \Gamma
$$

where the sign $\sim$ means that it holds in some basis obtained from that of (C.4) by a simultaneous permutation of rows and columns. From this expression, one can see that

$n_{21}$ has no row or column with more than two $1 \mathrm{~s}$,

$n_{21}$ has exactly $n$ rows and columns with one 1 ,

properties invariant under permutations of rows and columns.

We also know that $n_{21}$ must commute with $n_{12}$. In a basis in which $n_{12}$ takes the form (C.4), $n_{21}$ may thus be regarded as made of $n \times n$ blocks that commute with $G$. We shall combine these facts about $n_{21}$ as follows:

- A non-vanishing matrix $X$ with elements in $\mathbb{N}$ which commutes with $G$ cannot have a row or a column of zeros. Proof: let $\psi^{1}$ be the Perron-Frobenius eigenvector of $G$, $G X=X G$ implies that $X \psi^{1}$ is an eigenvector of $G$ with the same eigenvalue, hence proportional to $\psi^{1}, X \psi^{1}=c \psi^{1}$, with $c \neq 0$ since the entries of both $X$ and of $\psi^{1}$ are non-negative. If $X$ had a vanishing row, $X \psi^{1}$ would have a vanishing component, which is impossible for the Perron-Frobenius eigenvector. If $X$ has a vanishing column, one repeats the argument with $X^{T}$.

- Any matrix $X$ with elements in $\mathbb{N}$ which commutes with $G$ and which appears in the block decomposition of $n_{21}$ cannot have more than one 1 per row or column. Proof: If 
a matrix $X$ with more than one 1 in a row (respectively column) was a block of $n_{21}$, because of the property (C.6), all the other blocks on the left or the right (respectively above or below) of $X$ would have to have at least one vanishing row (respectively column), which is impossible by (i) above, or to vanish altogether. In the latter case, after a possible reshuffling of rows and columns leaving (C.4) invariant, one would have

$$
\text { either } n_{21}=\left(\begin{array}{cccc}
X & 0 & \cdots & 0 \\
0 & & & \\
\vdots & & & \\
0 & & &
\end{array}\right) \quad \text { or } \quad n_{21}=\left(\begin{array}{cccc}
0 & X & 0 & \cdots \\
X^{T} & 0 & 0 & \cdots \\
0 & 0 & & \\
\vdots & \vdots & &
\end{array}\right)
$$

which would lead to a pair $n_{12}, n_{21}$ reducible in the same basis.

- It follows that the matrices that may appear as blocks in the decomposition of $n_{21}$ must be matrices with one 1 on each row and column, i.e. permutation matrices that commute with $G$. These permutation matrices are the symmetries of the Dynkin diagram, and thus are readily listed:

$$
\begin{aligned}
& X=\quad I, \Gamma \quad \text { if } G=A_{n}, D_{2 q+1}, E_{6} \\
& =I, \Gamma_{i}, i=1, \cdots, 5 \quad \text { if } G=D_{4} \\
& =\quad I, \Gamma^{\prime} \quad \text { if } G=D_{2 q}, q>2 \\
& =\quad I \quad \text { if } G=E_{7}, E_{8}
\end{aligned}
$$

Here $\Gamma_{i}$ denote the 5 non-trivial permutations of the nodes of the $D_{4}$ diagrams, and the matrix $\Gamma^{\prime}$ exchanges the two end points of $D_{2 q}, q>2$.

One then demands that the symmetric matrix $n_{21}$ made of such blocks is irreducible and satisfies (C.4)-(C.7). This implies that at most one non-vanishing block appears on the diagonal. Consistency with the form $n_{21} \sim T_{p} \otimes \Gamma$ leaves as the only possibility

$$
n_{21}=\left(\begin{array}{ccccc}
0 & X_{1} & 0 & \cdots & \\
X_{1}^{T} & 0 & X_{2} & 0 & \\
0 & X_{2}^{T} & \ddots & \ddots & \\
& 0 & \ddots & & X_{p-1} \\
0 & & & X_{p-1}^{T} & \Gamma
\end{array}\right)
$$

where $X_{1}, X_{2}, \ldots, X_{p-1}$ are chosen among the symmetry matrices of $G$. A final permutation of rows and columns by a block diagonal matrix $\operatorname{diag}\left(Y_{1}, Y_{2}, \cdots, Y_{p-1}, I\right)$ brings $n_{21}$ into the form $n_{21}=T_{p} \otimes \Gamma$ while leaving the form (C.4) of $n_{12}$ unchanged, provided $Y_{j}=$ $\Gamma Y_{j+1} X_{j}^{T}$, hence $Y_{j}=\Gamma^{p-j} X_{p-1}^{T} X_{p-2}^{T} \cdots X_{j}^{T}$. Then both $n_{12}$ and $n_{21}$ have their canonical forms (C.1),(C.3). Q.E.D 


\section{Remark}

Although it is not required for the present analysis, it may be interesting to look at the commutant of matrices of $A-D-E$ type.

For any $G$ of $A-D-E$ type, with the exception of $D_{\text {even }}$, all eigenvalues are distinct. It follows that any matrix $X$ that commutes with $G$ may be diagonalized in the same basis as $G$ and consequently be written as a polynomial of $G$, i.e. as a linear combination of $I, G, G^{2}, \cdots, G^{n-1}$. In order to look at cases where entries of $X$ are requested to take values 0 or 1 only, and with constraints on the number of 1 's, it is advantageous to use rather the basis of fused graph matrices: $X$ is a linear combination of the linearly independent matrices $\hat{N}_{1}=I, \hat{N}_{2}=G, \cdots, \hat{N}_{n}$. The $D_{\text {even }}$ case is slightly more involved, since the matrices that appear naturally are not independent.

The commutant of an $A-D-E$ matrix is:

- A linear combination of the graph fusion matrices $\hat{N}_{a}$ for $G=A, D_{\text {odd }}, E_{6}, E_{7}, E_{8}$.

- A linear combination of the $\hat{N}_{a}$ and of two of the three matrices $\Sigma_{a b}, a \neq b=1,3,4$, that exchange two of the three extremal points of the $D_{4}$ graph.

- A linear combination of the $\hat{N}_{a}$ and of the two matrices $X=\Gamma^{\prime} \hat{N}_{2 q}$ and $Y=\hat{N}_{2 q} \Gamma^{\prime}$, where the matrix $\Gamma^{\prime}$ exchanges the two end points, for $G=D_{2 q}, q>2$.

\section{Appendix D: $s \widehat{l(3)}$ Modular Invariants and Graphs}

The WZW sl(3) theories may be discussed along the same lines as in Sections 2 and 3. Solutions $n_{i}$ to the Cardy equation are associated with graphs, with specific spectral properties: their eigenvalues are given by ratios of elements of the modular $S$ matrix labelled by weights of the diagonal spectrum $\mathcal{E}$ of the bulk theory. Conversely, spectral properties and the fact the $n$ 's form a representation of the fusion algebra are not restrictive enough to yield the list of possible bulk spectra, as occurred in $\widehat{s(2)}$ (up to the unwanted "tadpole" graphs). There are indeed many solutions, i.e. graphs and representations of the fusion algebra, that must be discarded as not corresponding to a modular invariant partition function in the list (Table 2) of Gannon [81]: see [39] for such extra solutions. We may summarise the salient features of the analysis as follows, see also the accompanying Tables 2 and 3 and Figures 11 and 12 .

- At least one graph (or rather one set of $n$ matrices) has been identified for each bulk theory, i.e. each modular invariant. But it is not known if this list of graphs and n's is exhaustive.

- Note that the hypothesis of 3-colourability of the graphs that looked natural on the basis of the $s l(2)$ case has to be abandonned if we want to cover all cases. This is 
manifest on Table 3 where it appears that in some cases (namely $A^{(n) *}$, and $D^{(n)}, n$ not a multiple of 3 , and $E^{(8) *}$, the set $\mathcal{E}$ is not invariant under the automorphism $\sigma$ of (C.1), as it should be if the graph was 3-colourable.

- There are a few pairs or even triplets of isospectral graphs, i.e. different sets of $n$ 's that give distinct solutions of the Cardy equation for a given bulk theory. These graphs/representations should not only describe different sets of complete orthonormal sets of boundary conditions for that bulk theory, but also presumably be associated with different operator algebras and lattice realisations.

- In Table 3, which summarises the state of the art, we have also indicated if the graph is of type I or type II, following the discussion of Section 3. Some hybrid cases are also encountered, in which the $M$ and $\hat{N}$ structure constants are both non-negative, but the $\hat{N}$ algebra has no subalgebra isomorphic to some extended fusion algebra.

\section{Notations and footnotes for Tables 2 and 3}

(Shifted) weights of $S U(3) \lambda=\left(\lambda_{1}, \lambda_{2}\right):=\lambda_{1} \Lambda_{1}+\lambda_{2} \Lambda_{2}$, where $\Lambda_{1}, \Lambda_{2}$ are the fundamental weights of $S U(3), \lambda^{*}=\left(\lambda_{2}, \lambda_{1}\right)$, triality $\tau(\lambda):=\left(\lambda_{1}-1\right)+2\left(\lambda_{2}-1\right) \equiv \lambda_{1}-\lambda_{2} \quad \bmod 3$.

$Q$ is the set of weights of triality zero.

Weyl alcove of shifted level, or "altitude", $n:=k+3$,

$P_{++}^{(n)}=\left\{\Lambda=\lambda_{1} \Lambda_{1}+\lambda_{2} \Lambda_{2} \mid \lambda_{1}, \lambda_{2} \geq 1, \lambda_{1}+\lambda_{2} \leq n-1\right\}$.

Automorphism $\sigma$ of $P_{++}^{(n)}$

$$
\sigma\left(\lambda_{1}, \lambda_{2}\right):=\left(n-\lambda_{1}-\lambda_{2}, \lambda_{1}\right)
$$

(a). One of the two connected parts of the fused Dynkin diagram of type $A_{n-1}$ (the one that possesses the exponent $(1,1))$. It looks different depending on whether $n$ is even or odd. The $M, \hat{N}$ algebras of $\mathcal{A}^{(n) *}$ are positive, as they follow simply from the Verlinde fusion algebra $N$ of $\widehat{s l}(2)$. If $n$ is odd, $\mathcal{A}^{(n) *}$ is the connected component of the graph of adjacency matrix $A_{n-1}^{2}-I$ made of the nodes $a$ odd (integer spin in $s l(2)$ ). For a triplet of "exponents" $\lambda=(l, l), \mu=(m, m)$ and $\rho=(r, r)$ of $\mathcal{A}^{(n) *}, M_{\lambda, \mu}{ }^{\rho}=N_{l m}{ }^{r}+N_{l m}{ }^{n-r}$, and $\hat{N}_{a b}{ }^{c}$ is the restriction of the Verlinde $A_{n-1}$ algebra to odd $a, b, c$. For even $n$, the $M$ and $\hat{N}$ algebras of $\mathcal{A}^{(n) *}=I+A_{\frac{n}{2}-1}$ coincide with the Verlinde algebra of $A_{\frac{n}{2}-1}$.

(b). The orbifold of $\mathcal{A}^{(n)}$, see [62].

(c). The ordinary $\mathbb{Z}_{3}$ fold of $\mathcal{A}^{(n)}$.

(d). The unfolded (and 3-colourable) version of $\mathcal{A}^{(n) *}$. Their adjacency matrix is a tensor product by the permutation matrix $\sigma_{123}=\left(\begin{array}{ccc}0 & 1 & 0 \\ 0 & 0 & 1 \\ 1 & 0 & 0\end{array}\right)$; their $M$ and $\hat{N}$ algebras are simply obtained from those of $\mathcal{A}^{(n) *}$, thus also $\geq 0$

(e). The $\mathbb{Z}_{3}$ fold of $\mathcal{E}^{(8)}$. 
Table 2. List of $\widehat{s l}(3)_{k}$ modular invariants; $n=k+3$.

$$
\begin{aligned}
& \left(A^{(n)}\right) \quad Z=\sum_{\lambda \in P_{++}^{(n)}}\left|\chi_{\lambda}\right|^{2} \\
& \left(A^{(n) *}\right) \quad Z=\sum_{\lambda \in P_{++}^{(n)}} \chi_{\lambda} \chi_{\lambda^{*}}{ }^{*}
\end{aligned}
$$

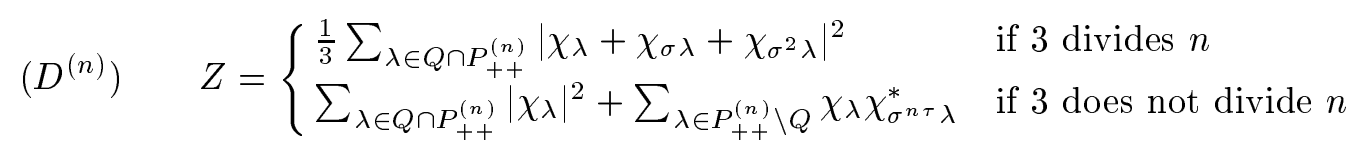

$$
\begin{aligned}
& \left(D^{(n) *}\right) \quad Z= \begin{cases}\frac{1}{3} \sum_{\lambda \in Q \cap P_{++}^{(n)}}\left(\sum_{\ell=0}^{2} \chi_{\sigma^{\ell} \lambda}\right)\left(\sum_{\ell=0}^{2} \chi_{\sigma^{\ell} \lambda^{*}}\right)^{*} & \text { if } 3 \text { divides } n \\
\sum_{\lambda \in Q \cap P_{++}^{(n)}} \chi_{\lambda} \chi_{\lambda^{*}}+\sum_{\lambda \in P_{++}^{(n)} \backslash Q} \chi_{\lambda} \chi_{\sigma^{-n \tau} \lambda^{*}}^{*} & \text { if } 3 \text { does not divide } n\end{cases} \\
& \left(E^{(8)}\right) \quad Z=\left|\chi_{(1,1)}+\chi_{(3,3)}\right|^{2}+\left|\chi_{(3,2)}+\chi_{(1,6)}\right|^{2}+\left|\chi_{(2,3)}+\chi_{(6,1)}\right|^{2}+\left|\chi_{(4,1)}+\chi_{(1,4)}\right|^{2}+\left|\chi_{(1,3)}+\chi_{(4,3)}\right|^{2}+\left|\chi_{(3,1)}+\chi_{(3,4)}\right|^{2} \\
& \left(E^{(8) *}\right) \quad Z=\left|\chi_{(1,1)}+\chi_{(3,3)}\right|^{2}+\left(\chi_{(3,2)}+\chi_{(1,6)}\right)\left(\chi_{(2,3)}+\chi_{(6,1)}\right)^{*}+\text { c.c. }+\left|\chi_{(4,1)}+\chi_{(1,4)}\right|^{2}+\left(\chi_{(1,3)}+\chi_{(4,3)}\right)\left(\chi_{(3,1)}+\chi_{(3,4)}\right)^{*}+\text { c.c. } \\
& \left(E^{(12)}\right) \quad Z=\left|\chi_{(1,1)}+\chi_{(10,1)}+\chi_{(1,10)}+\chi_{(5,5)}+\chi_{(5,2)}+\chi_{(2,5)}\right|^{2}+2\left|\chi_{(3,3)}+\chi_{(3,6)}+\chi_{(6,3)}\right|^{2} \\
& \left(E_{M S}^{(12)}\right) \quad Z=\left|\chi_{(1,1)}+\chi_{(10,1)}+\chi_{(1,10)}\right|^{2}+\left|\chi_{(3,3)}+\chi_{(3,6)}+\chi_{(6,3)}\right|^{2}+\left|\chi_{(5,5)}+\chi_{(5,2)}+\chi_{(2,5)}\right|^{2}+\left|\chi_{(4,7)}+\chi_{(7,1)}+\chi_{(1,4)}\right|^{2} \\
& +\left|\chi_{(7,4)}+\chi_{(1,7)}+\chi_{(4,1)}\right|^{2}+2\left|\chi_{(4,4)}\right|^{2}+\left(\chi_{(2,2)}+\chi_{(8,2)}+\chi_{(2,8)}\right) \chi_{(4,4)}^{*}+\text { c.c. } \\
& \left(E_{M S}^{(12) *}\right) \quad Z=\left|\chi_{(1,1)}+\chi_{(10,1)}+\chi_{(1,10)}\right|^{2}+\left|\chi_{(3,3)}+\chi_{(3,6)}+\chi_{(6,3)}\right|^{2}+\left|\chi_{(5,5)}+\chi_{(5,2)}+\chi_{(2,5)}\right|^{2}+2\left|\chi_{(4,4)}\right|^{2} \\
& +\left(\chi_{(4,7)}+\chi_{(7,1)}+\chi_{(1,4)}\right)\left(\chi_{(7,4)}+\chi_{(1,7)}+\chi_{(4,1)}\right)^{*}+\text { c.c. }+\left(\chi_{(2,2)}+\chi_{(8,2)}+\chi_{(2,8)}\right) \chi_{(4,4)}^{*}+\text { c.c. } \\
& \left(E^{(24)}\right) \quad Z=\left|\chi_{(1,1)}+\chi_{(22,1)}+\chi_{(1,22)}+\chi_{(5,5)}+\chi_{(5,14)}+\chi_{(14,5)}+\chi_{(11,11)}+\chi_{(11,2)}+\chi_{(2,11)}+\chi_{(7,7)}+\chi_{(7,10)}+\chi_{(10,7)}\right|^{2} \\
& +\left|\chi_{(7,1)}+\chi_{(16,7)}+\chi_{(1,16)}+\chi_{(1,7)}+\chi_{(7,16)}+\chi_{(16,1)}+\chi_{(5,8)}+\chi_{(11,5)}+\chi_{(8,11)}+\chi_{(8,5)}+\chi_{(5,11)}+\chi_{(11,8)}\right|^{2}
\end{aligned}
$$


Table 3. List of modular invariants and associated known graphs for $\widehat{s l}(3)$ Footnotes (a)-(e) are explained at the end of Appendix D.

\begin{tabular}{|c|c|c|c|c|}
\hline Modular Invariant & Graphs & Altitude & Exponents & Type \\
\hline$A^{(n)}$ & $\mathcal{A}^{(n)}$ & $n$ & $P_{++}^{(n)}$ & $I$ \\
\hline$A^{(n) *}$ & $\mathcal{A}^{(n)}=\left(A_{n-1} \star A_{n-1}\right)_{c}$ & $n$ & $\{(j, j)\} \quad 1 \leq j \leq\left\lfloor\frac{n-1}{2}\right\rfloor$ & $M, \hat{N} \geq 0$ \\
\hline$D^{(n)}$ & $\begin{array}{cc}\mathcal{D}^{(n)} & (b) \\
\mathcal{A}^{(n)} / \mathbb{Z}_{3} & (c) \\
\end{array}$ & $\begin{array}{l}n=0 \bmod 3 \\
n \neq 0 \bmod 3\end{array}$ & $\begin{array}{l}P_{++}^{(n)} \cap Q, \text { with }\left(\frac{n}{3}, \frac{n}{3}\right) \text { triple } \\
P_{++}^{(n)} \cap Q\end{array}$ & $\begin{array}{c}I \\
M, \hat{N} \geq 0\end{array}$ \\
\hline$D^{(n) *}$ & $\mathcal{D}^{(n)}=\sigma_{123} \otimes\left(A_{n-1} \star A_{n-1}\right)_{c}$ & $n$ & $\begin{array}{l}\{(j, j),(n-2 j, j),(j, n-2 j)\} \\
1 \leq j \leq\left\lfloor\frac{n-1}{2}\right\rfloor\end{array}$ & $M, \hat{N} \geq 0$ \\
\hline$E^{(8)}$ & $\mathcal{E}^{(8)}$ & 8 & $\begin{array}{l}(1,1),(6,1),(1,6),(3,3),(3,2),(2,3), \\
(4,1),(3,4),(1,3),(1,4)(4,3),(3,1)\end{array}$ & $I$ \\
\hline$E^{(8) *}$ & $\mathcal{E}^{(8) *} \quad(e)$ & 8 & $(1,1),(3,3),(4,1),(1,4)$ & $M, \hat{N} \geq 0$ \\
\hline$E^{(12)}$ & $\mathcal{E}_{i}^{(12)}, i=1,2,3$ & 12 & $\begin{array}{l}(1,1),(10,1),(1,10),(5,5),(5,2),(2,5), \\
\text { and twice }(3,3),(3,6),(6,3)\end{array}$ & $\begin{array}{c}\mathcal{E}_{i}^{(12)}, i=1,3: I \\
\mathcal{E}_{2}^{(12)} I I\end{array}$ \\
\hline$E_{\mathrm{MS}}^{(12) *}$ & $\mathcal{E}_{4}^{(12)}$ & 12 & $\begin{array}{l}(1,1),(10,1),(1,10),(5,5),(5,2),(2,5) \\
(3,3),(3,6),(6,3) \text { and twice }(4,4)\end{array}$ & $I I$ \\
\hline$E_{\mathrm{MS}}^{(12)}$ & $\mathcal{E}_{5}^{(12)}$ & 12 & $\begin{array}{l}(1,1),(10,1),(1,10),(5,5),(5,2),(2,5), \\
(4,1),(7,4),(1,7),(1,4),(7,1),(4,7), \\
(3,3),(3,6),(6,3) \text { and twice }(4,4)\end{array}$ & $I I$ \\
\hline$E^{(24)}$ & $\mathcal{E}^{(24)}$ & 24 & $\begin{array}{l}(1,1),(22,1),(1,22),(5,5),(14,5),(5,14), \\
(7,7),(10,7),(7,10),(11,11),(11,2),(2,11), \\
(7,1),(16,7),(1,16),(1,7),(16,1),(7,16), \\
(5,8),(11,5),(8,11),(8,5),(11,8),(5,11)\end{array}$ & $I$ \\
\hline
\end{tabular}



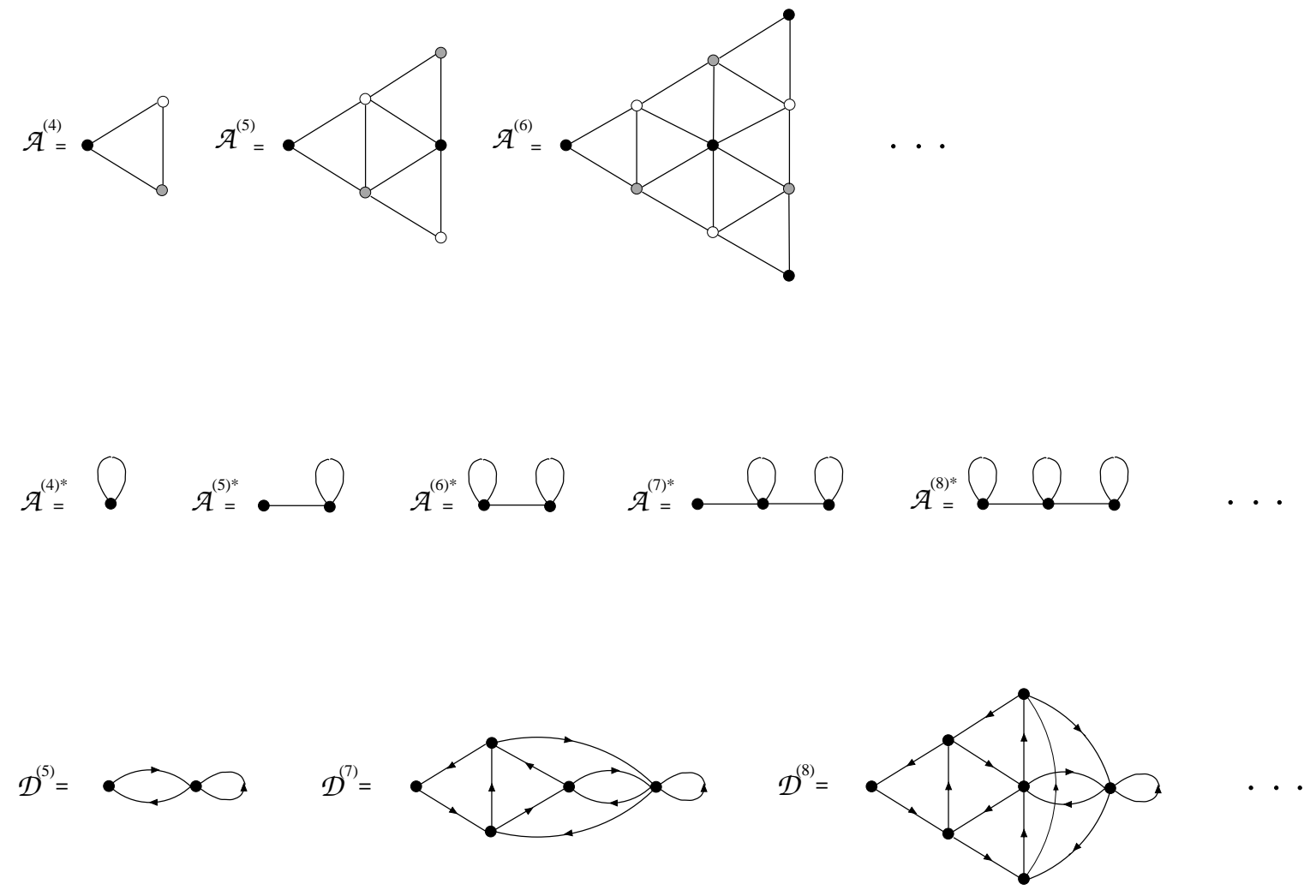

$\mathcal{D}^{(6)}=$
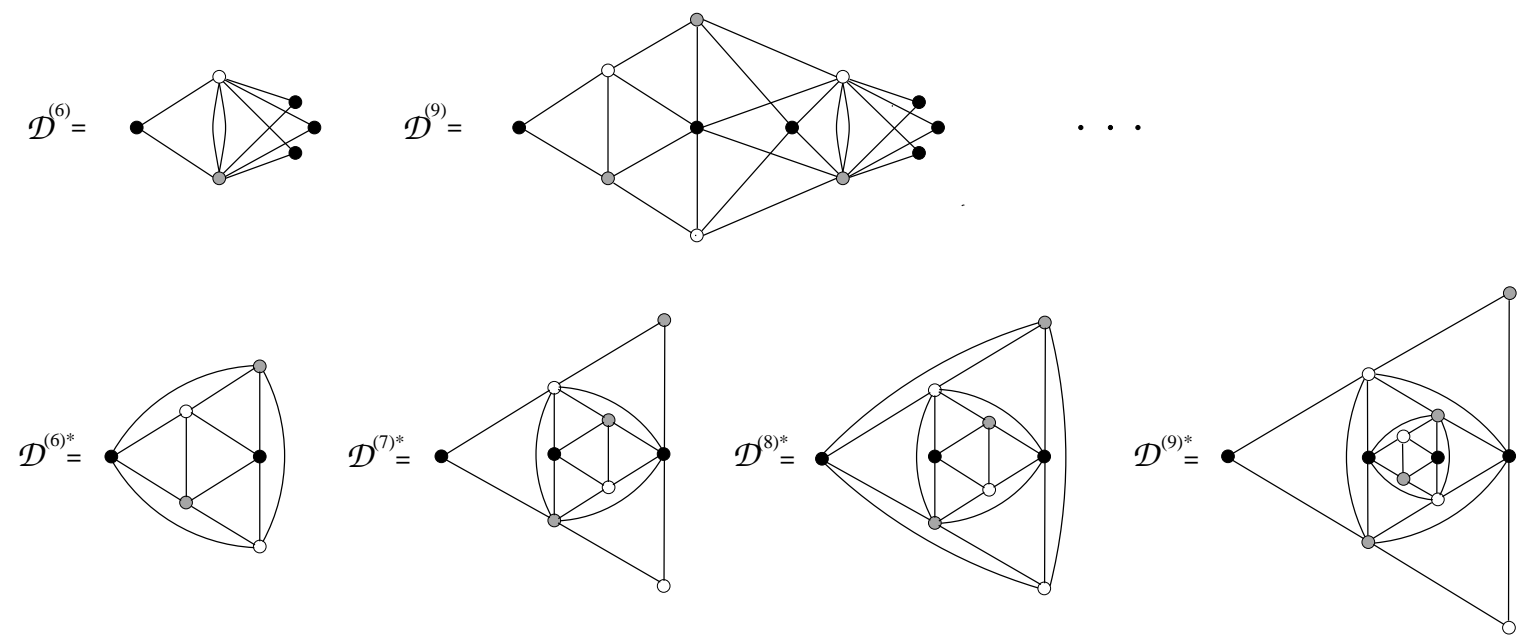

Figure 11: The known graphs in the case of $\widehat{s l}(3)$. Conventions : (a) For the 3-colourable graphs, the triality $\tau$ of nodes is indicated by the colour: black $\tau=0$, grey $\tau=1$, or white $\tau=2$; the graph represents the matrix $n_{21}$ if edges are oriented from black to grey, or grey to white, etc. (b) For the non-3-colourable graphs, either the orientation of all edges (of matrix $n_{21}$, say) is indicated, $\left(\mathcal{D}^{(n)}\right.$, $3 \not n$, series, $\left.\mathcal{E}^{(8) *}\right)$, or all links are unoriented $\left(\mathcal{A}^{(\cdot) *}\right.$ series $)$. 

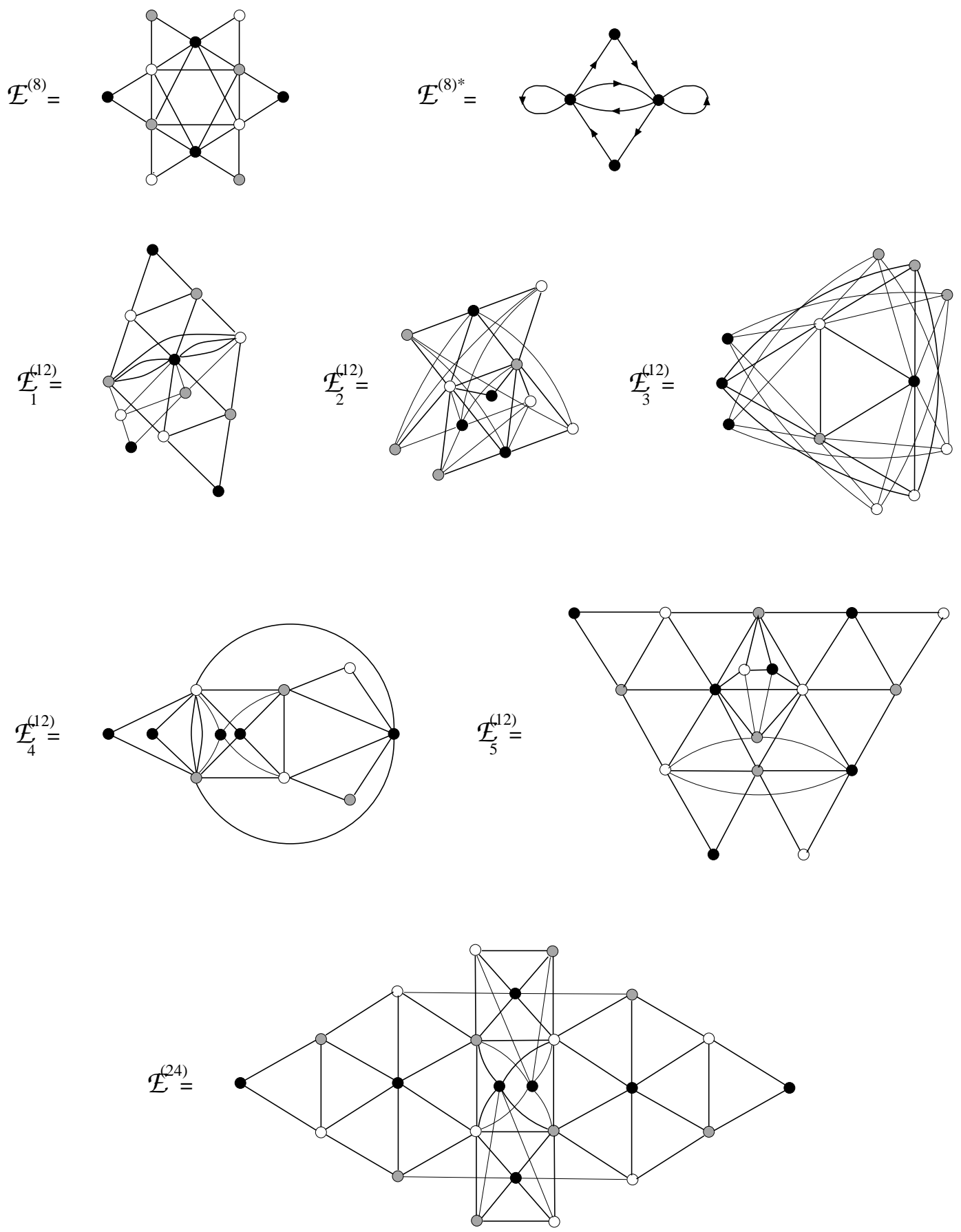

Figure 12: The known graphs in the case of $\widehat{s l}(3)$, (cont'd). 


\section{Appendix E: Formulae for fusing, braiding and modular matrices}

We collect in this appendix some standard formulae for the genus 0 and 1 duality matrices. The fusing matrices satisfy several identities implied by the pentagon identity - they can be recovered from formulae (4.36), (4.38), (4.16) in the text making the identification (4.54).

Symmetries:

$$
F_{p q}\left[\begin{array}{cc}
k & j \\
i & l
\end{array}\right]=F_{p^{*} q}\left[\begin{array}{cc}
j & k \\
l^{*} & i^{*}
\end{array}\right]=F_{p q^{*}}\left[\begin{array}{cc}
i^{*} & l \\
k^{*} & j
\end{array}\right]=F_{p^{*} q^{*}}\left[\begin{array}{ll}
l & i^{*} \\
j^{*} & k
\end{array}\right] .
$$

Choice of gauge:

$$
F_{q 1}^{(0)}\left[\begin{array}{cc}
i & i^{*} \\
j & j
\end{array}\right]_{\alpha \beta}^{\mathbf{1}_{j} \mathbf{1}_{i}^{\prime}}=\sqrt{\frac{d_{q}}{d_{i} d_{j}}} \delta_{\beta \sigma_{13}\left(\alpha^{*}\right)}
$$

In the $\operatorname{sl}(2)$ case denote by $\sqrt{C_{k j}^{q}}$ the normalisation of the CVO in this gauge. Then for the fusion matrix corresponding to CVO normalised to 1, one has

$$
F_{p q}\left[\begin{array}{cc}
k & j \\
i & l
\end{array}\right]=\sqrt{\frac{C_{k j}^{q} C_{q l}^{i}}{C_{k p}^{i} C_{j l}^{p}}} F_{p q}^{(0)}\left[\begin{array}{cc}
k & j \\
i & l
\end{array}\right]=\sqrt{\frac{C_{k j}^{q} C_{i^{*} l}^{q^{*}} C_{q q^{*}}^{1}}{C_{k i^{*}}^{p^{*}} C_{j l}^{p} C_{p p^{*}}^{1}}} F_{p q}^{(0)}\left[\begin{array}{cc}
k & j \\
i & l
\end{array}\right],
$$

or,

$$
C_{k i^{*}}^{p^{*}} C_{j l}^{p} C_{p p^{*}}^{1} F_{p q}\left[\begin{array}{cc}
k & j \\
i & l
\end{array}\right]=C_{k j}^{q} C_{i^{*} l}^{q^{*}} C_{q q^{*}}^{1} F_{q p}\left[\begin{array}{cc}
k^{*} & i \\
j & l^{*}
\end{array}\right] .
$$

This equation coincides with the quadratic relation resulting from locality of the physical 4point function in the diagonal case. Hence the constants $C_{k p}^{i}=C_{(k, k)(p, p)}^{(i, i}, C_{k p}^{i} C_{i i^{*}}^{1}=C_{k i^{*}}^{p^{*}} C_{p p^{*}}^{1}$ can be identified with the physical OPE structure constants in this case. For the minimal models these constants were computed in [69]; the matrices $F^{(0)}$ in the gauge $(\mathbb{E} .2)$ coincide up to signs with a product of standard $q-6 j$ symbols, see 85 for the latter.

Braiding matrices:

$$
B_{p q}\left[\begin{array}{ll}
i & j \\
k & l
\end{array}\right]_{\beta_{1} \sigma_{23}\left(\beta_{2}\right)}^{\sigma_{23}\left(\gamma_{2}\right) \delta}(\epsilon)=e^{\pi i \epsilon\left(\triangle_{k}+\triangle_{l}-\triangle_{p}-\triangle_{q}\right)} F_{p q}\left[\begin{array}{ll}
i & l \\
k & j
\end{array}\right]_{\beta_{1} \beta_{2}}^{\gamma_{2} \delta}
$$

The $q$ - analogs of the Racah identity (hexagon identities), $\epsilon= \pm 1$ :

$$
\sum_{q} F_{m q}\left[\begin{array}{cc}
i & k \\
j & l
\end{array}\right] e^{-\pi i \epsilon \triangle_{q}} F_{q p}\left[\begin{array}{ll}
l & i \\
j & k
\end{array}\right]=e^{\pi i \epsilon\left(\triangle_{m}+\triangle_{p}-\triangle_{l}-\triangle_{j}-\triangle_{i}-\triangle_{k}\right)} F_{m p}\left[\begin{array}{ll}
i & l \\
j & k
\end{array}\right] .
$$

Recall the Moore-Seiberg torus duality identity resulting from a relation in the modular group of the torus with two field insertions, namely, $S\left(j_{1}, j_{2}\right) a=b S\left(j_{1}, j_{2}\right)$ where $S\left(j_{1}, j_{2}\right)$ is the modular matrix of two-point blocks, expressed in terms of $F$ and $S(p)$, and $a, b$ are 
the monodromy transformations moving one of the CVO around the $a, b$ cycles, 33.

$$
\begin{array}{r}
S_{r i}(s) \sum_{m} e^{2 \pi i\left(\triangle_{i}-\triangle_{m}\right)} F_{s^{*} m}\left[\begin{array}{cc}
j_{2} & i \\
j_{1}^{*} & i^{*}
\end{array}\right] F_{m p}\left[\begin{array}{cc}
j_{1} & j_{2} \\
i & i
\end{array}\right] \\
=\sum_{q} S_{q i}(p) e^{\pi i\left(\triangle_{p}-\triangle_{j_{1}}-\triangle_{j_{2}}\right)} F_{s q^{*}}\left[\begin{array}{cc}
r^{*} & j_{1} \\
r^{*} & j_{2}
\end{array}\right] F_{r p}\left[\begin{array}{cc}
j_{2} & j_{1} \\
q & q
\end{array}\right] .
\end{array}
$$

Choose $s=1=r$. This implies that $j_{1}=j_{2}^{*}=j^{*}$ and $q=j$, hence

$$
\begin{aligned}
S_{j i}(p) & =\frac{S_{1 i}}{F_{1 p}\left[\begin{array}{cc}
j & j^{*} \\
j & j
\end{array}\right]} \sum_{m} e^{\pi i\left(2 \triangle_{i}+2 \triangle_{j}-2 \triangle_{m}-\triangle_{p}\right)} F_{1 m}\left[\begin{array}{ll}
j & i \\
j & i^{*}
\end{array}\right] F_{m p}\left[\begin{array}{cc}
j^{*} & j \\
i & i
\end{array}\right] \\
& =\frac{S_{1 i}}{F_{1 p}\left[\begin{array}{cc}
j & j^{*} \\
j & j
\end{array}\right]} \sum_{m} e^{-\pi i\left(2 \triangle_{i}+2 \triangle_{j}-2 \triangle_{m}\right)} F_{1 m}\left[\begin{array}{cc}
j^{*} & i \\
j^{*} & i^{*}
\end{array}\right] F_{m p}\left[\begin{array}{cc}
j & j^{*} \\
i & i
\end{array}\right] \\
& =\frac{S_{1 j}}{F_{p 1}\left[\begin{array}{cc}
i^{*} & i \\
i^{*} & i^{*}
\end{array}\right]} \sum_{m} e^{\pi i\left(2 \triangle_{i}+2 \triangle_{j}-2 \triangle_{m}-\triangle_{p}\right)} F_{p m}\left[\begin{array}{cc}
j & i \\
j & i^{*}
\end{array}\right] F_{m 1}\left[\begin{array}{cc}
j^{*} & j \\
i & i
\end{array}\right] .
\end{aligned}
$$

The second equality is obtained reversing the sums in (E.7) and solving for $S_{r i}(s)$ as above, while the third is obtained from the transposed version of (E.7) taking into account $S(p)^{2}=$ $C e^{-\pi i \triangle_{p}}$. For $p=1$ the formula reproduces the ordinary $S=S(1)$ matrix

$$
\begin{aligned}
S_{i j} & =\frac{S_{i 1}}{F_{11}\left[\begin{array}{cc}
j & j^{*} \\
j & j
\end{array}\right]} \sum_{m} e^{2 \pi i\left(\triangle_{i}+\triangle_{j}-\triangle_{m}\right)} F_{1 m}\left[\begin{array}{cc}
j & i \\
j & i^{*}
\end{array}\right] F_{m 1}\left[\begin{array}{cc}
j^{*} & j \\
i & i
\end{array}\right] \\
& =S_{11} \sum_{m} e^{2 \pi i\left(\triangle_{i}+\triangle_{j}-\triangle_{m}\right)} d_{m} N_{i j}^{m} .
\end{aligned}
$$

\section{Acknowledgements}

P.A.P. is supported by the Australian Research Council. V.B.P. acknowledges the hospitality of Arnold Sommerfeld Institute for Mathematical Physics, TU Clausthal, the support and hospitality of Service de Physique Théorique, CEA-Saclay and the partial support of the Bulgarian National Research Foundation (contract $\Phi-643)$. J.-B.Z. acknowledges partial support of the EU Training and Mobility of Researchers Program (Contract FMRX-CT960012), which made possible an extremely profitable stay in SISSA, Trieste. We acknowledge useful discussions with M. Bauer, D. Bernard, J. Fuchs, T. Gannon, A. Honecker, A. Recknagel, P. Ruelle, I. Runkel, C. Schweigert and G. Watts.

\section{References}

[1] Kawai, H., Lewellen, D.C. and Tye, S.-H., Nucl. Phys. B 269 (1986) 1-23, A relation between tree amplitudes of closed and open strings 
[2] Ishibashi, N., Mod. Phys. Lett. A 4 (1987) 251-264, The Boundary and Crosscap States in Conformal Field Theories

[3] Ishibashi, N. and Onogi, T., Mod. Phys. Lett. A 4 (1987) 161-168, Conformal field theories on surfaces with boundaries and cross-caps

[4] Callan, C.G., Lovelace, C., Nappi, C. and Yost, S.A., Nucl. Phys. B 293 (1987) 83-113, Adding holes and crosscaps to the superstring

[5] Bern, Z. and Dunbar, D., Phys. Lett. B 203 (1988) 109-117, Coupling of open to closed bosonic strings in four dimensions

[6] Polchinski, J. and Cai, Y., Nucl. Phys. B 296 (1988) 91-128, Consistency of open superstring theories

[7] Pradisi, G. and Sagnotti, A., Phys. Lett. B 216 (1989) 59-67, Open string orbifolds

[8] Bianchi, M. and Sagnotti, A., Phys. Lett. B 247 (1990) 517-524, On the systematics of open string theories

[9] Saleur, H. and Bauer, M., Nucl. Phys. B 320 (1989) 591-624, On some relations between local height probabilities and conformal invariance

[10] Cardy, J.L., Nucl. Phys. B 324 (1989) 581-596, Boundary Conditions, Fusion Rules and the Verlinde Formula

[11] Cardy, J.L. and Lewellen, D. C., Phys. Lett. B 259 (1991) 274-278, Bulk and boundary operators in conformal field theory

[12] Lewellen, D. C., Nucl. Phys. B 372 (1992) 654-682, Sewing constraints for conformal field theories on surfaces with boundaries

[13] Pradisi, G., Sagnotti, A. and Stanev, Ya.S., Phys. Lett. B 356 (1995) 230-238, The Open Descendants of Non-Diagonal SU(2) WZW Models

[14] Pradisi, G., Sagnotti, A. and Stanev, Ya.S., Phys. Lett. B 381 (1996) 97-104, Completeness conditions for boundary operators in $2 D$ conformal field theory

[15] Sagnotti, A. and Stanev, Ya.S., Fortschr. Phys. 44 (1996) 585-596, Open descendants in conformal field theory

[16] Affleck, I. and Ludwig, A., Phys. Rev. Lett. 67 (1991) 161-164, Universal Noninteger "Ground-State Degeneracy" in Critical Quantum Systems 
[17] Affleck, I., Conformal Field Theory Approach to the Kondo Effect, in Bosonization and Conformal Symmetry in High Energy Physics and Condensed Matter Physics, Zakopane, (1995) (M.A. Nowak and P. Wegrzyn, Act. Phys. Pol. 26 (1995), 1869-1932) and further references therein

[18] Cherednik, I.V., Teor. Mat. Fiz. 61 (1894) 35-44, Factorizing Particles on a Half-Line and Root Systems

[19] Ghoshal, S. and Zamolodchikov, A., Int. J. Mod. Phys. A 9 (1994) 3841-3886, Err. ibid, 4353, Boundary $S$ matrix and boundary state in two-dimensional Integrable Quantum Field Theory

[20] Corrigan, E., Dorey, P.E., Rietdijk, R.H., and Sasaki, R., Phys. Lett. B 333 (1994) 83-91, hep-th 9404108, Affine Toda Field Theory on a half-line

[21] Oshikawa, M. and Affleck, I., Nucl. Phys. B 495 (1997) 533-582, Boundary conformal field theory approach to the critical two-dimensional Ising model with a defect line

[22] Affleck, I., Oshikawa, M. and Saleur, H., J. Phys. A 31 (1998) 5827-5842, cond-mat 9804117, Boundary Critical Phenomena in the Three-State Potts Model

[23] Sklyanin, E.K., J. Phys. A 21 (1988) 2375-2389, Boundary Conditions for Integrable Quantum Systems

[24] Behrend, R. E., Pearce, P. A. and O'Brien, D. L., J. Stat. Phys. 84 (1996) 1-48, Interaction-Round-a-Face Models with Fixed Boundary Conditions: The ABF Fusion Hierarchy; Behrend, R. E. and Pearce, P. A., J. Phys. A29 (1996) 7827-7835, A Construction of Solutions to Reflection Equations for Interaction-Round-a-Face Models

[25] Recknagel, A. and Schomerus, V., Nucl. Phys. B 531 (1998) 185-225, D-branes in Gepner models

[26] Recknagel, A. and Schomerus, V., Nucl. Phys. B 545 (1999) 233-282, Boundary deformation theory and moduli spaces of D-Branes

[27] Fuchs, J. and Schweigert, C., Nucl. Phys. B 530 (1998) 99-136, Branes; From Free Fields to General Backgrounds; Phys. Lett. 414 (1997) 251-259, A classifying algebra for boundary conditions

[28] Fuchs, J. and Schweigert, C., Phys. Lett. B 441 (1998) 141-146, Completeness of Boundary Conditions for the Critical Three-State Potts Model

[29] Fuchs, J. and Schweigert, C., Phys. Lett. B 447 (1999) 266-276, Orbifold analysis of broken bulk symmetries; hep-th/9902132, Symmetry breaking boundaries I. General Theory; Birke, L., Fuchs, J. and Schweigert, C., hep-th/9905038, Symmetry breaking boundary conditions and WZW orbifolds 
[30] Sagnotti, A. and Stanev, Ya.S. in DESY meeting on Conformal field theory of D-branes (Hamburg, 7-12 Sept., 1998)

[31] Behrend, R. E., Pearce, P. A. and Zuber, J.-B., J. Phys. A 31 (1998) L 763-770, hep-th/9807142, Integrable boundaries, conformal boundary conditions and $A-D-E$ fusion rules

[32] Behrend, R. E., Pearce, P. A., Petkova, V., and Zuber, J.-B., , Phys. Lett. B 444 (1998) 163-166, On the classification of bulk and boundary conformal field theories

[33] Moore, G. and Seiberg, N., , Comm. Math. Phys. 123 (1989) 177-254, Classical and Quantum Conformal Field Theory; Lectures on RCFT, Physics, Geometry and Topology, Plenum Press, New York, 1990

[34] Verlinde, E., Nucl. Phys. B 300 (1988) 360-376, Fusion Rules and Modular Transformations in 2-D Conformal Field Theory

[35] Sonoda, H., Nucl. Phys. B 281 (1987) 546-572, The energy-momentum tensor on a Riemann surface; ibid. B284 (1987) 157-192, Functional determinants on a punctured Riemann surface and their application to string theory

[36] Cardy, J.L., Nucl. Phys. B 240 [FS12] (1984) 514-532, Conformal invariance and surface critical behavior; ibid B 275 [FS17] (1986) 200-218, Effect of boundary conditions on the operator content of two-dimensional conformally invariant theories

[37] Kato, M., Okada, T., Nucl. Phys. B 499 (1997) 583-595, D-Branes on Group Manifolds

[38] Kac, V., Infinite dimensional Lie algebras (Cambridge Univ. Pr., 1995)

[39] Di Francesco, P. and Zuber, J.-B., Nucl. Phys. B 338 (1990) 602-646, SU(N) Lattice Integrable Models Associated with Graphs

[40] Goodman, F. M., de la Harpe, P. and Jones, V. F. R., Coxeter Dynkin diagrams and towers of algebras ( Vol. 14, MSRI Publications, Springer, Berlin, 1989)

[41] Cappelli, A., Itzykson, C. and Zuber, J.-B., Nucl. Phys. B 280 (1987) 445-465, Modular Invariant Partition Functions in Two Dimensions; Comm. Math. Phys. 113 (1987) 126, The A-D-E Classification of Minimal and $A_{1}^{(1)}$ Conformal Invariant Theories; Kato, A., Mod. Phys. Lett. A 2 (1987) 585-600, Classification of Modular Invariant Partition Functions in Two Dimensions

[42] Gel'fand, I.M., Graev, M.I. and Vilenkin, N.Ya, Generalised functions, v.5, ch. VII (Academic Press, New York, 1966) 
[43] Andrews, G. E., Baxter, R. J. and Forrester, P. J., J. Stat. Phys. 35 (1984) 193-266, Eight-Vertex SOS Model and Generalized Rogers-Ramanujan-Type Identities

[44] Pasquier, V., Nucl. Phys. B 285 (1987) 162-172, Two-Dimensional Critical Systems Labelled by Dynkin Diagrams

[45] Roche, P., Phys. Lett. B 285 (1992) 49-53, On the Construction of Integrable Dilute A-D-E Models

[46] Warnaar, S. O. and Nienhuis, B., J. Phys. A 26 (1993) 2301-2316, Solvable Lattice Models Labelled by Dynkin Diagrams

[47] Forrester, P. and Baxter, R., J. Stat. Phys. 38 (1885) 435-472, Further Exact Solutions of the Eight-Vertex SOS Model and Generalizations of Rogers-Ramanujan Identities

[48] Pearce, P. A. and Zhou, Y.-K., Int. J. Mod. Phys. B 7 (1993) 3649-3705, Intertwiners and $A-D-E$ Lattice Models

[49] Pasquier, V. and Saleur, H., Nucl. Phys. B 330 (1990) 523-556, Common structures between finite size systems and conformal field theories through quantum groups

[50] Pasquier, V., J. Phys. A 20 (1987) 5707-5717, Operator Content of the ADE lattice models; and the preprint version of that article, reprinted in V. Pasquier, Modèles Exacts Invariants Conformes, Thèse d'Etat, Orsay, 1988.

[51] Di Francesco, P. and Zuber, J.-B. SU(N) Lattice Integrable Models and Modular Invariance in Recent Developments in Conformal Field Theories Trieste Conference, (1989, S. Randjbar-Daemi, E. Sezgin and J.-B. Zuber eds., World Scientific 1990); Di Francesco, P., Int. J. Mod. Phys. A7 (1992) 407-500, Integrable Lattice Models , Graphs and Modular Invariant Conformal Field Theories

[52] Petkova, V.B. and Zuber, J.-B., Nucl. Phys. B 438 (1995) 347-372, On structure constants of $\operatorname{sl}(2)$ theories

[53] Petkova, V.B. and Zuber, J.-B., Nucl. Phys. B 463 (1996) 161-193, From CFT to Graphs

[54] Petkova, V.B. and Zuber, J.-B., hep-th preprint 9701103, Conformal Field Theory and Graphs

[55] Xu, F., Comm. Math. Phys. 192 (1998) 349-403, New braided endomorphisms from conformal inclusions

[56] Böckenhauer J. and Evans, D. E., Comm. Math. Phys. 200 (1999) 57-103, Modular invariants, graphs and $\alpha$-induction for nets of subfactors II; Modular invariants, graphs and $\alpha$-induction for nets of subfactors III, hep-th/9812110 
[57] Böckenhauer J., Evans, D. E. and Kawahigashi, Y., math.OA preprint 9904109, On $\alpha$-induction, chiral generators and modular invariants for subfactors

[58] Honecker, A., Nucl. Phys. B 400 (1993) 574-596, Automorphisms of $\mathcal{W}$-algebras and extended rational conformal field theories

[59] Zuber, J.-B., Generalized Dynkin diagrams and root systems, in Topological Field Theory, Primitive Forms and Related Topics Taniguchi Symposium, Kyoto, 1996 ( M. Kashiwara, A. Matsuo, K. Saito and I. Satake eds, , Birkhäuser 1998., )

[60] Itzykson, C., Nucl. Phys. Proc. Suppl. 5B (1988) 150-165, Level one Kac-Moody Characters and Modular Invariance

[61] Degiovanni, P., Comm. Math. Phys. 127 (1990) 71-99, $\mathbb{Z} / N \mathbb{Z}$ conformal field theories

[62] Kostov, I., Nucl. Phys. B 300 (1988) 559-587, Free field presentation of the $A_{n}$ coset models on the torus

[63] Dijkgraaf, R., Vafa, C., Verlinde, E. and Verlinde, H., Comm. Math. Phys. 123 (1989) 485-526, Operator algebra of orbifold models

[64] Di Francesco, P., Mathieu, P. and Sénéchal, D., Conformal Field Theory (Springer, 1997)

[65] Böhm, G. and Szlachanyi, K., , Lett. Math. Phys. 38 (1996) 437-456, A coassociative $C^{*}$ - quantum group with non-integral dimensions, q-alg/9509008

[66] Roche P., Comm. Math. Phys. 127 (1990) 395-424, Ocneanu Cell Calculus and Integrable Lattice Models

[67] Y.-K. Zhou and P. A. Pearce, Int. J. Mod. Phys. B 8 (1994) 3531-3577, Fusion of ADE lattice models

[68] Zamolodchikov, A.B. and Fateev, V.A., Sov. J. Nucl. Phys. 43 (1986) 657-664, Operator algebra and correlation functions in the two-dimensional $S U(2) \times S U(2)$ Wess-Zumino model

[69] Dotsenko, Vl.S. and Fateev, V.A., Nucl. Phys. B 251[FS13] (1985) 691-734, Fourpoint correlation functions and the operator algebra in $2 d$ conformal invariant theories with central charge $c \leq 1$

[70] Runkel, I., Nucl. Phys. B 549 (1999) 563-578, Boundary structure constants for the A-series Virasoro minimal models

[71] Bannai, E., and Ito, T., Algebraic Combinatorics I: Association Schemes, (BenjaminCummings, 1984) 
[72] Bántay, P. and Vecsernyés, P., , Int. J. Mod. Phys. A 14 (1999) 1325-, Mapping class group representations and generalised Verlinde formula, hep-th/9506186

[73] Ocneanu, A., Paths on Coxeter Diagrams: From Platonic Solids and Singularities to Minimal Models and Subfactors in Low Dimensional Topology, Statistical Mechanics and Quantum Field Theory Fields Institute, Waterloo, Ontario, April 26-30, 1995. (Notes taken by S. Goto, , )

[74] Dorey, P., Int. J. Mod. Phys. A 8 (1993) 193-208, Partition Functions, Intertwiners and the Coxeter Element

[75] McKay, J., Proc. Symp. Pure Math. 37 (1980) 183-186, Graphs, Singularities, and Finite Groups

[76] Kostant, B., Astérisque (Société Mathématique de France), (1988) 209-255, The McKay Correspondence, the Coxeter Element and Representation Theory

[77] Braden, H., Corrigan, E., Dorey, P. and Sasaki, R., Nucl. Phys. B 338 (1990) 689-746, Affine Toda Field Theory and Exact $S$ Matrices

[78] McCoy, B.M., and Orrick, W., Phys. Lett. A 230 (1997) 24-32, Single Particle Excitations in the Lattice $E_{8}$ Ising Model

[79] Batchelor, M.T. and Seaton, K.A., cond-mat preprint 9803206, Excitations in the diluate $A_{L}$ lattice model: $E_{6}, E_{7}$ and $E_{8}$ mass spectra

[80] Suzuki, J., cond-mat preprint 9805241, Quantum Jacobi-Trudi Formula and $E_{8}$ Structure in the Ising Model in a Field

[81] Gannon, T., Comm. Math. Phys. 161 (1994) 233-263, The Classification of Affine SU(3) Modular Invariant Partition Functions; Ann. Inst. H. Poincaré, Phys.Theor. 65 (1996) 15-56, hep-th/9404185, The classification of SU(3) modular invariant revisited,

[82] Runkel, I., hep-th preprint 9908046, Structure constants for the D-series Virasoro minimal models

[83] Ruelle, P., hep-th preprint 9904100, Symmetric boundary conditions in boundary critical phenomena

[84] Di Francesco, P. and Zuber, J.-B., (unpublished), reported in the second reference [51, see also [74

[85] Kirillov, A.N. and Reshetikhin, N.Yu., in New developments of the theory of knots (T. Kohno ed. , World Sc. 1990., )

[86] Böckenhauer J., Evans, D. E. and Kawahigashi, Y., math.OA preprint 9907143, Chiral structure of modular invariants for subfactors 